\title{
Systemic safety of bevacizumab versus ranibizumab for neovascular age-related macular degeneration (Review)
}

Moja L, Lucenteforte E, Kwag KH, Bertele V, Campomori A, Chakravarthy U, D’Amico R, Dickersin K, Kodjikian L, Lindsley K, Loke Y, Maguire M, Martin DF, Mugelli A, Mühlbauer B, Püntmann I, Reeves B, Rogers C, Schmucker C, Subramanian ML, Virgili G

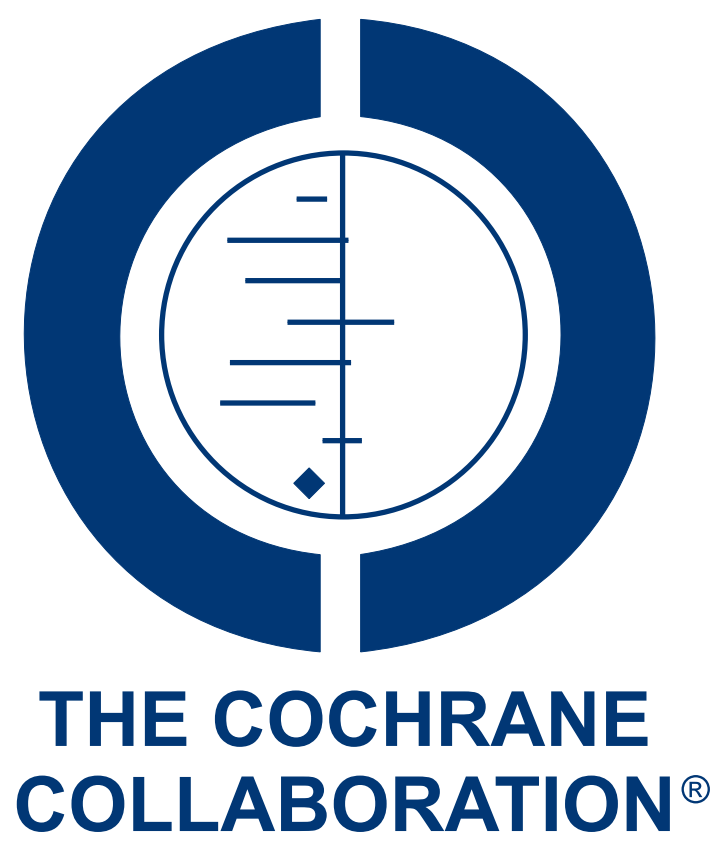

This is a reprint of a Cochrane review, prepared and maintained by The Cochrane Collaboration and published in The Cochrane Library 2014, Issue 9

http://www.thecochranelibrary.com

\section{WILEY}

Systemic safety of bevacizumab versus ranibizumab for neovascular age-related macular degeneration (Review) Copyright $\odot 2014$ The Cochrane Collaboration. Published by John Wiley \& Sons, Ltd. 
TABLE OF CONTENTS

HEADER . . . . . . . . . . . . . . . . . . . . . . . . . . . . . . . . . . . . . . . . . . . . . . . . . . . .

ABSTRACT . . . . . . . . . . . . . . . . . . . . . . . . . . . . . . . . . . . . . . . . . . . . . . . . . . .

PLAIN LANGUAGE SUMMARY . . . . . . . . . . . . . . . . . . . . . . . . . . . . . . . . . . . . . . . . . . . . . . . .

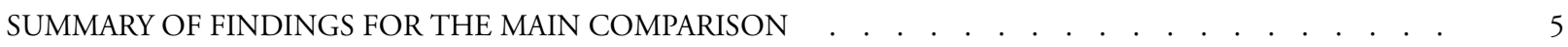

BACKGROUND . . . . . . . . . . . . . . . . . . . . . . . . . . . . . . . . . . . . . . . . . . . . . . .

OBJECTIVES . . . . . . . . . . . . . . . . . . . . . . . . . . . . . . . . . . . . . . . . . . .

METHODS . . . . . . . . . . . . . . . . . . . . . . . . . . . . . . . . . . . . . . . . .

RESULTS . . . . . . . . . . . . . . . . . . . . . . . . . . . . . . . . . . . . . . . . . . . .

Figure 1. . . . . . . . . . . . . . . . . . . . . . . . . . . . . . . . . . . . . . . .

Figure 2. . . . . . . . . . . . . . . . . . . . . . . . . . . . . . . . . . . . . . 13

Figure 3. . . . . . . . . . . . . . . . . . . . . . . . . . . . . . . . . . . . . 15

Figure $4 . \quad$. . . . . . . . . . . . . . . . . . . . . . . . . . . . . . . . . . . . . . . .

DISCUSSION . . . . . . . . . . . . . . . . . . . . . . . . . . . . . . . . . . . . . . . . . . . . .

AUTHORS' CONCLUSIONS . . . . . . . . . . . . . . . . . . . . . . . . . . . . . . . . . . . . . . . . .

ACKNOWLEDGEMENTS . . . . . . . . . . . . . . . . . . . . . . . . . . . . . . . . . . . . . . . . . 19

REFERENCES . . . . . . . . . . . . . . . . . . . . . . . . . . . . . . . . . . . . . . . . . . . .

CHARACTERISTICS OF STUDIES . . . . . . . . . . . . . . . . . . . . . . . . . . . . . . . . . . . . . .

DATA AND ANALYSES . . . . . . . . . . . . . . . . . . . . . . . . . . . . . . . . . . . . . . . . . . 49

Analysis 1.1. Comparison 1 Bevacizumab versus ranibizumab, longest follow-up, Outcome 1 All-cause death. . . . . 51

Analysis 1.2. Comparison 1 Bevacizumab versus ranibizumab, longest follow-up, Outcome 2 All serious systemic adverse events.

Analysis 1.3. Comparison 1 Bevacizumab versus ranibizumab, longest follow-up, Outcome 3 Arterial thromboembolic event.

Analysis 1.4. Comparison 1 Bevacizumab versus ranibizumab, longest follow-up, Outcome 4 Gastrointestinal perforation.

Analysis 1.5. Comparison 1 Bevacizumab versus ranibizumab, longest follow-up, Outcome 5 Infection. . . . . . . 55

Analysis 1.6. Comparison 1 Bevacizumab versus ranibizumab, longest follow-up, Outcome 6 Myocardial infarction. . 56

Analysis 1.7. Comparison 1 Bevacizumab versus ranibizumab, longest follow-up, Outcome 7 Neutropenia. . . . $\quad$. 57

Analysis 1.8. Comparison 1 Bevacizumab versus ranibizumab, longest follow-up, Outcome 8 Non-ocular haemorrhage. 57

Analysis 1.9. Comparison 1 Bevacizumab versus ranibizumab, longest follow-up, Outcome 9 Stroke. . . . . . . $\quad$. 58

Analysis 1.11. Comparison 1 Bevacizumab versus ranibizumab, longest follow-up, Outcome 11 Vascular events associated with anti-VEGF treatment. . . . . . . . . . . . . . . . . . . . . . . . . . . . . . 59

Analysis 1.12. Comparison 1 Bevacizumab versus ranibizumab, longest follow-up, Outcome 12 Cardiac disorders MedDRA Class.

Analysis 1.13. Comparison 1 Bevacizumab versus ranibizumab, longest follow-up, Outcome 13 Gastrointestinal disorders MedDRA Class. . . . . . . . . . . . . . . . . . . . . . . . . . . . . . . . . . .

Analysis 1.14. Comparison 1 Bevacizumab versus ranibizumab, longest follow-up, Outcome 14 General disorders and administration site conditions MedDRA Class.

Analysis 1.15. Comparison 1 Bevacizumab versus ranibizumab, longest follow-up, Outcome 15 Neoplasms benign, malignant, and unspecified MedDRA Class.

Analysis 1.16. Comparison 1 Bevacizumab versus ranibizumab, longest follow-up, Outcome 16 Nervous system disorders MedDRA Class.

Analysis 1.17. Comparison 1 Bevacizumab versus ranibizumab, longest follow-up, Outcome 17 Respiratory, thoracic, and mediastinal disorders MedDRA Class.

Analysis 2.1. Comparison 2 Bevacizumab versus ranibizumab, one-year of follow-up, primary outcomes, Outcome 1 Allcause death.

Analysis 2.2. Comparison 2 Bevacizumab versus ranibizumab, one-year of follow-up, primary outcomes, Outcome 2 All serious systemic adverse events.

Analysis 3.1. Comparison 3 Bevacizumab versus ranibizumab, influence analysis: all SSAEs excluding CATT, Outcome 1 All serious systemic adverse events.

Analysis 4.1. Comparison 4 Bevacizumab versus ranibizumab, influence analysis: all SSAEs excluding LUCAS, Outcome 1 All serious systemic adverse events. 
Analysis 5.1. Comparison 5 Bevacizumab versus ranibizumab, influence analysis: primary outcomes excluding unpublished studies, Outcome 1 All-cause death.

Analysis 5.2. Comparison 5 Bevacizumab versus ranibizumab, influence analysis: primary outcomes excluding unpublished studies, Outcome 2 All serious systemic adverse events.

Analysis 6.1. Comparison 6 Bevacizumab versus ranibizumab, secondary analysis: fixed-effect analyses, Outcome 1 Allcause death.

Analysis 6.2. Comparison 6 Bevacizumab versus ranibizumab, secondary analysis: fixed-effect analyses, Outcome 2 All serious systemic adverse events. . . . . . . . . . . . . . . . . . . . . . . . . . . . . . . . . . . . .

Analysis 6.3. Comparison 6 Bevacizumab versus ranibizumab, secondary analysis: fixed-effect analyses, Outcome 3 Arterial thromboembolic event. . . . . . . . . . . . . . . . . . . . . . . . . . . . . . . . . . .

Analysis 6.4. Comparison 6 Bevacizumab versus ranibizumab, secondary analysis: fixed-effect analyses, Outcome 4 Gastrointestinal perforation. . . . . . . . . . . . . . . . . . . . . . . . . . . . . .

Analysis 6.5. Comparison 6 Bevacizumab versus ranibizumab, secondary analysis: fixed-effect analyses, Outcome 5 Infections.

Analysis 6.6. Comparison 6 Bevacizumab versus ranibizumab, secondary analysis: fixed-effect analyses, Outcome 6 Myocardial infarction. . . . . . . . . . . . . . . . . . . . . . . . . . . . . . . .

Analysis 6.7. Comparison 6 Bevacizumab versus ranibizumab, secondary analysis: fixed-effect analyses, Outcome 7 Neutropenia. . . . . . . . . . . . . . . . . . . . . . . . . . . . . . . . . . . .

Analysis 6.8. Comparison 6 Bevacizumab versus ranibizumab, secondary analysis: fixed-effect analyses, Outcome 8 Non-

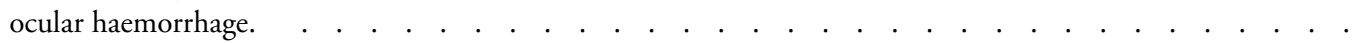

Analysis 6.9. Comparison 6 Bevacizumab versus ranibizumab, secondary analysis: fixed-effect analyses, Outcome 9 Stroke.

Analysis 6.11. Comparison 6 Bevacizumab versus ranibizumab, secondary analysis: fixed-effect analyses, Outcome 11 Vascular events associated with anti-VEGF treatment.

Analysis 6.12. Comparison 6 Bevacizumab versus ranibizumab, secondary analysis: fixed-effect analyses, Outcome 12 Cardiac disorders MedDRA Class. . . . . . . . . . . . . . . . . . . . . . . . . . . .

Analysis 6.13. Comparison 6 Bevacizumab versus ranibizumab, secondary analysis: fixed-effect analyses, Outcome 13 Gastrointestinal disorders MedDRA Class. . . . . . . . . . . . . . . . . . . . . . . . .

Analysis 6.14. Comparison 6 Bevacizumab versus ranibizumab, secondary analysis: fixed-effect analyses, Outcome 14 General disorders and administration site conditions MedDRA Class.

Analysis 6.15. Comparison 6 Bevacizumab versus ranibizumab, secondary analysis: fixed-effect analyses, Outcome 15 Neoplasms benign, malignant, and unspecified MedDRA Class.

Analysis 6.16. Comparison 6 Bevacizumab versus ranibizumab, secondary analysis: fixed-effect analyses, Outcome 16 Nervous system disorders MedDRA Class. . . . . . . . . . . . . . . . . . . . . . . . . . . . . . . . . . . . .

Analysis 6.17. Comparison 6 Bevacizumab versus ranibizumab, secondary analysis: fixed-effect analyses, Outcome 17 Respiratory, thoracic, and mediastinal disorders MedDRA Class. . . . . . . . . . . . . . . . . .

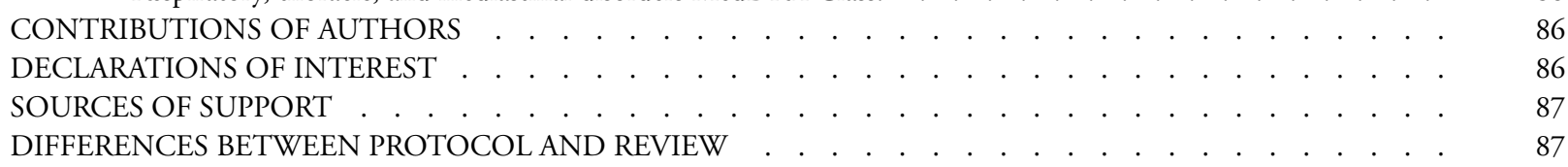

Systemic safety of bevacizumab versus ranibizumab for neovascular age-related macular degeneration (Review) 


\section{[Intervention Review]}

\section{Systemic safety of bevacizumab versus ranibizumab for neovascular age-related macular degeneration}

Lorenzo Moja ${ }^{1}$, Ersilia Lucenteforte ${ }^{2}$, Koren $\mathrm{H} \mathrm{Kwag}^{3}$, Vittorio Bertele ${ }^{4}$, Annalisa Campomori ${ }^{5}$, Usha Chakravarthy ${ }^{6}$, Roberto D’Amico $^{7}$, Kay Dickersin ${ }^{8}$, Laurent Kodjikian ${ }^{9}$, Kristina Lindsley ${ }^{10}$, Yoon Loke ${ }^{11}$, Maureen Maguire ${ }^{12}$, Daniel F Martin ${ }^{13}$, Alessandro Mugelli $^{14}$, Bernd Mühlbauer ${ }^{15}$, Isabel Püntmann ${ }^{15}$, Barnaby Reeves ${ }^{16}$, Chris Rogers ${ }^{16}$, Christine Schmucker ${ }^{17}$, Manju L Subramanian 18, Gianni Virgili ${ }^{19}$

${ }^{1}$ Department of Biomedical Sciences for Health, University of Milan - IRCCS Galeazzi Orthopaedic Institute, Milan, Italy. ${ }^{2}$ Department of Neurosciences, Psychology, Drug Research and Children's Health, University of Florence, Florence, Italy. ${ }^{3}$ Clinical Epidemiology Unit, IRCCS Galeazzi Orthopaedic Institute, Milan, Italy. ${ }^{4}$ Laboratory of Regulatory Policies, IRCCS Mario Negri Institute for Pharmacological Research, Milan, Italy. ${ }^{5}$ Hospital Pharmacy, Trento General Hospital, Health Trust of the Autonomous Province of Trento, Trento, Italy. ${ }^{6}$ Centre for Vision and Vascular Science, Queen's University Belfast, Belfast, UK. ${ }^{7}$ Italian Cochrane Centre, Department of Diagnostic, Clinical and Public Health Medicine, University of Modena and Reggio Emilia, Modena, Italy. ${ }^{8}$ Center for Clinical Trials and US Cochrane Center, Johns Hopkins University, Baltimore, MD, USA. ${ }^{9}$ Department of Ophthalmology, Hôpital de la Croix-Rousse, Lyon, France. ${ }^{10}$ Department of Epidemiology, Johns Hopkins Bloomberg School of Public Health, Baltimore, Maryland, USA. ${ }^{11}$ School of Medicine, University of East Anglia, Norwich, UK. ${ }^{12}$ Department of Ophthalmology, University of Pennsylvania, Philadelphia, Pennsylvania, USA. ${ }^{13}$ Cole Eye Institute, Cleveland Clinic, Cleveland, Ohio, USA. ${ }^{14}$ Department of Neurosciences, Psychology, Drug Research and Child Health, University of Florence, Florence, Italy. ${ }^{15}$ Dept of Pharmacology, Klinikum Bremen Mitte gGmbH, Bremen, Germany. ${ }^{16}$ School of Clinical Sciences, University of Bristol, Bristol, UK. ${ }^{17}$ German Cochrane Centre, Institute of Medical Biometry and Medical Informatics, University Medical Center Freiburg, Freiburg, Germany. ${ }^{18}$ Department of Ophthalmology, Boston University, School of Medicine, Boston, Massachusetts, USA. ${ }^{19}$ Department of Translational Surgery and Medicine, Eye Clinic, University of Florence, Florence, Italy

Contact address: Lorenzo Moja, Department of Biomedical Sciences for Health, University of Milan - IRCCS Galeazzi Orthopaedic Institute, Via Pascal 36, Milan, 20133, Italy. lorenzo.moja@unimi.it.

Editorial group: Cochrane Eyes and Vision Group.

Publication status and date: New, published in Issue 9, 2014.

Review content assessed as up-to-date: 27 March 2014.

Citation: Moja L, Lucenteforte E, Kwag KH, Bertele V, Campomori A, Chakravarthy U, D’Amico R, Dickersin K, Kodjikian L, Lindsley K, Loke Y, Maguire M, Martin DF, Mugelli A, Mühlbauer B, Püntmann I, Reeves B, Rogers C, Schmucker C, Subramanian ML, Virgili G. Systemic safety of bevacizumab versus ranibizumab for neovascular age-related macular degeneration. Cochrane Database of Systematic Reviews 2014, Issue 9. Art. No.: CD011230. DOI: 10.1002/14651858.CD011230.pub2.

Copyright (C) 2014 The Cochrane Collaboration. Published by John Wiley \& Sons, Ltd.

\section{A B S T R A C T}

\section{Background}

Neovascular age-related macular degeneration (AMD) is the leading cause of legal blindness in elderly populations of industrialised countries. Bevacizumab (Avastin $\left.{ }^{\circledR}\right)$ and ranibizumab (Lucentis $\left.{ }^{\circledR}\right)$ are targeted biological drugs (a monoclonal antibody) that inhibit vascular endothelial growth factor, an angiogenic cytokine that promotes vascular leakage and growth, thereby preventing its pathological angiogenesis. Ranibizumab is approved for intravitreal use to treat neovascular AMD, while bevacizumab is approved for intravenous use as a cancer therapy. However, due to the biological similarity of the two drugs, bevacizumab is widely used off-label to treat neovascular AMD.

Systemic safety of bevacizumab versus ranibizumab for neovascular age-related macular degeneration (Review)

Copyright @ 2014 The Cochrane Collaboration. Published by John Wiley \& Sons, Ltd. 


\section{Objectives}

To assess the systemic safety of intravitreal bevacizumab (brand name Avastin ${ }^{\circledR}$; Genentech/Roche) compared with intravitreal ranibizumab (brand name Lucentis ${ }^{\circledR}$; Novartis/Genentech) in people with neovascular AMD. Primary outcomes were death and All serious systemic adverse events (All SSAEs), the latter as a composite outcome in accordance with the International Conference on Harmonisation Good Clinical Practice. Secondary outcomes examined specific SSAEs: fatal and non-fatal myocardial infarctions, strokes, arteriothrombotic events, serious infections, and events grouped in some Medical Dictionary for Regulatory Activities System Organ Classes (MedDRA SOC). We assessed the safety at the longest available follow-up to a maximum of two years.

\section{Search methods}

We searched CENTRAL, MEDLINE, EMBASE and other online databases up to 27 March 2014. We also searched abstracts and clinical study presentations at meetings, trial registries, and contacted authors of included studies when we had questions.

\section{Selection criteria}

Randomised controlled trials (RCTs) directly comparing intravitreal bevacizumab (1.25 mg) and ranibizumab (0.5 mg) in people with neovascular AMD, regardless of publication status, drug dose, treatment regimen, or follow-up length, and whether the SSAEs of interest were reported in the trial report.

\section{Data collection and analysis}

Two authors independently selected studies and assessed the risk of bias for each study. Three authors independently extracted data.

We conducted random-effects meta-analyses for the primary and secondary outcomes. We planned a pre-specified analysis to explore deaths and All SSAEs at the one-year follow-up.

\section{Main results}

We included data from nine studies (3665 participants), including six published (2745 participants) and three unpublished ( 920 participants) RCTs, none supported by industry. Three studies excluded participants at high cardiovascular risk, increasing clinical heterogeneity among studies. The studies were well designed, and we did not downgrade the quality of the evidence for any of the outcomes due to risk of bias. Although the estimated effects of bevacizumab and ranibizumab on our outcomes were similar, we downgraded the quality of the evidence due to imprecision.

At the maximum follow-up (one or two years), the estimated risk ratio (RR) of death with bevacizumab compared with ranibizumab was 1.10 (95\% confidence interval (CI) 0.78 to 1.57 , P value $=0.59$; eight studies, 3338 participants; moderate quality evidence) Based on the event rates in the studies, this gives a risk of death with ranibizumab of $3.4 \%$ and with bevacizumab of $3.7 \%$ (95\% CI $2.7 \%$ to $5.3 \%$ ).

For All SSAEs, the estimated RR was 1.08 (95\% CI 0.90 to 1.31, P value $=0.41$; nine studies, 3665 participants; low quality evidence). Based on the event rates in the studies, this gives a risk of SSAEs of $22.2 \%$ with ranibizumab and with bevacizumab of $24 \%$ (95\% CI $20 \%$ to $29.1 \%)$.

For the secondary outcomes, we could not detect any difference between bevacizumab and ranibizumab, with the exception of gastrointestinal disorders MedDRA SOC where there was a higher risk with bevacizumab (RR 1.82; 95\% CI 1.04 to 3.19 , P value = 0.04; six studies, 3190 participants).

Pre-specified analyses of deaths and All SSAEs at one-year follow-up did not substantially alter the findings of our review.

Fixed-effect analysis for deaths did not substantially alter the findings of our review, but fixed-effect analysis of All SSAEs showed an increased risk for bevacizumab (RR 1.12; 95\% CI 1.00 to 1.26, P value $=0.04$; nine studies, 3665 participants): the meta-analysis was dominated by a single study (weight $=46.9 \%)$.

The available evidence was sensitive to the exclusion of CATT or unpublished results. For All SSAEs, the exclusion of CATT moved the overall estimate towards no difference (RR 1.01 ; 95\% CI 0.82 to 1.25 , P value $=0.92$ ), while the exclusion of LUCAS yielded a larger RR, with more SSAEs in the bevacizumab group, largely driven by CATT (RR 1.19; 95\% CI 1.06 to 1.34 , P value $=0.004$ ). The exclusion of all unpublished studies produced a RR of 1.12 for death $(95 \%$ CI 0.78 to 1.62 , P value $=0.53$ ) and a RR of 1.21 for SSAEs $(95 \%$ CI 1.06 to 1.37 , P value $=0.004)$, indicating a higher risk of SSAEs in those assigned to bevacizumab than ranibizumab. 


\section{Authors' conclusions}

This systematic review of non-industry sponsored RCTs could not determine a difference between intravitreal bevacizumab and ranibizumab for deaths, All SSAEs, or specific subsets of SSAEs in the first two years of treatment, with the exception of gastrointestinal disorders. The current evidence is imprecise and might vary across levels of patient risks, but overall suggests that if a difference exists, it is likely to be small. Health policies for the utilisation of ranibizumab instead of bevacizumab as a routine intervention for neovascular AMD for reasons of systemic safety are not sustained by evidence. The main results and quality of evidence should be verified once all trials are fully published.

\section{PLAIN LANGUAGESUMMARY}

\section{Systemic (whole body) safety of bevacizumab versus ranibizumab for neovascular age-related macular degeneration}

\section{Background}

Neovascular age-related macular degeneration (AMD) is a progressive and chronic disease of the eye, and a leading cause of severe blindness in elderly populations. The disease is characterised by the abnormal growth of arteries and veins (neovascularisation) in the macula, a region of the retina (back portion of eye) responsible for central vision. Without treatment, the leakage of these blood vessels causes swelling and damage to the macula, resulting in a fibrous scar that impairs eyesight. Approximately one out of 10 people with neovascular AMD suffer legal blindness, accounting for $90 \%$ of all cases of severe vision loss due to AMD.

Therapies against neovascular AMD target new blood vessels. Bevacizumab (commercial name Avastin ${ }^{\circledR}$ ) and ranibizumab (Lucentis $\left.{ }^{\circledR}\right)$ are biological drugs that bind to and block the function of vascular endothelial growth factor (VEGF), a protein released by cells in the body that stimulates the growth and leakage of blood vessels. The two drugs, accordingly, inhibit the process of neovascularisation. Ranibizumab is approved to treat neovascular AMD by injection into the eye (intravitreal injection), while bevacizumab is approved as a cancer therapy by injection into the vein through the skin. The two drugs have similar chemical structures and the same mechanism of action. Although their benefits are equivalent, it has been hypothesised that the two drugs have different systemic safety profiles, such that one drug might cause more adverse events (harms) at the level of whole body compared to the other.

\section{Review question}

We evaluated whether the two drugs differed in terms of deaths or serious systemic adverse events (SSAEs) in people with neovascular AMD. The latter refers to medically related events that result in death, are life-threatening, require hospital admission or prolong hospital stay, or cause persistent or significant disability.

\section{Study characteristics}

We included nine randomised controlled trials (RCTs), none supported by industry, with 3665 participants directly comparing bevacizumab with ranibizumab. Six RCTs were completed and published, two RCTs were completed, but unpublished, and one was still in progress. We were able to include safety information from all trials, accessing both published and unpublished data.

Drugs were administered for up to two years according to continuous or discontinuous treatment. In the first, drugs were regularly administered, irrespective of the remission or progression of the disease; the latter involved 'as needed' (pro re nata, PRN) or 'treatand-extend' regimens in which the drug was injected less frequently as long as there was no recurrence of neovascular manifestations. Follow-up for adverse events occurred at regular intervals up to one or two years, irrespective of continuous or discontinuous treatment.

All studies used the approved dosage of ranibizumab $(0.5 \mathrm{mg})$ according to the 'Summary of Product Characteristics', and the dosage of bevacizumab most recommended by ophthalmologists for intravitreal injection $(1.25 \mathrm{mg})$.

Three studies excluded patients at high cardiovascular risk. However, four RCTs considered patients at different cardiovascular risks, representing a wide spectrum of risks and routine practice in hospital settings.

\section{Key results}

Our review found the systemic safety of bevacizumab for neovascular AMD to be similar to that of ranibizumab, except for gastrointestinal disorders, which was a part of a secondary analysis.

If 1000 people were treated with ranibizumab for one or two years, 34 would die. If treated instead with bevacizumab, between 27 and 53 of them would die. If 1000 people were treated with ranibizumab, 222 would experience one or more SSAEs. If 1000 people

Systemic safety of bevacizumab versus ranibizumab for neovascular age-related macular degeneration (Review)

Copyright @ 2014 The Cochrane Collaboration. Published by John Wiley \& Sons, Ltd. 
were treated instead with bevacizumab, between 200 and 291 would experience such an event. Deaths are likely to be unrelated to the administration of drugs.

\section{Quality of the evidence}

We could not fully assess the quality of three unpublished studies. We rated the overall quality of the evidence as low to moderate because we could not be certain that one drug was better than the other one on many of our outcomes. Another limitation of the studies was the participants who were recruited into them, and the fact that studies may have missed measuring the outcomes of interest in a few individuals that might have experienced a SSAE. Missing information was equally common in participants treated with bevacizumab and those treated with ranibizumab. 


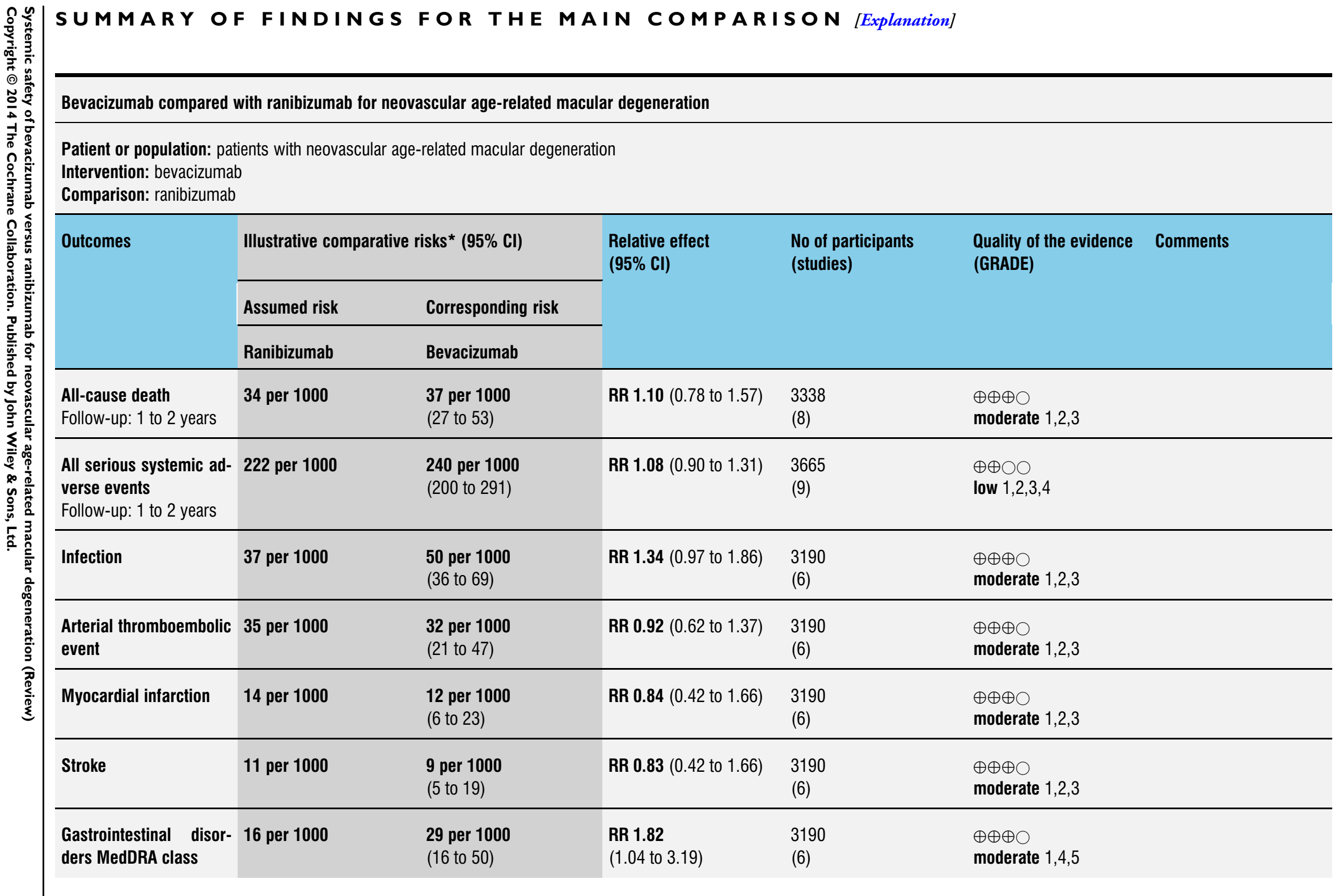


*The basis for the assumed risk (e.g., the median control group risk across studies) is provided in footnotes. The corresponding risk (and its $95 \%$ confidence interval) is based on the assumed risk in the comparison group and the relative effect of the intervention (and its $95 \% \mathrm{Cl}$ ).

Cl: confidence interval; RR: risk ratio

GRADE Working Group grades of evidence

High quality: Further research is very unlikely to change our confidence in the estimate of effect.

Moderate quality: Further research is likely to have an important impact on our confidence in the estimate of effect and may change the estimate.

Low quality: Further research is very likely to have an important impact on our confidence in the estimate of effect and is likely to change the estimate.

Very low quality: We are very uncertain about the estimate.

${ }^{1}$ For all outcomes, we judged the impact of missing data to be unclear: even the low rate of efficacy missing data, which was in the range of $5 \%$ to $10 \%$ for most studies, could have impacted on rare adverse events differences. However, we considered that a number of studies used an active monitoring for almost all participants across both arms, reducing the chance of missing SSAEs. We did not decrease the overall quality for risk of bias.

2People at high cardiovascular risk were excluded in Biswas 2011, MANTA, and Subramanian 2010. BRAMD, CATT, GEFAL, and IVAN did not report exclusion criteria based on cardiovascular risks, and we considered them to represent a wide spectrum of risks as well as routine practice in secondary care settings. However, it is unclear as to how the results can be applied to high-risk patients requiring long-term treatment. We did not decrease the overall quality for indirectness.

${ }^{3}$ Wide $95 \%$ confidence interval considering relative risk and/or absolute events. We did decrease the overall quality for imprecision (-1).

${ }^{4}$ The meta-analytic estimate was sensitive to the exclusion of CATT and LUCAS, as well as two unpublished studies. We did decrease

the overall quality for inconsistency (-1).

5'Summary of findings' table outcome not pre-specified in the review protocol. 


\section{B A C K G R O U N D}

\section{Description of the condition}

Age-related macular degeneration (AMD) is a progressive and chronic disease of the retina. According to numerous populationbased studies including individuals aged 30 to 97 years, the pooled prevalence of AMD at any stage is $8.7 \%$ (95\% credible interval (CrI) $4.3 \%$ to $17.4 \%$ ) and the prevalence of late-stage AMD is $0.4 \%$ (CrI $0.2 \%$ to $0.8 \%$ ), with higher prevalence observed in $\mathrm{Eu}$ ropean versus Asian or African populations (e.g., late AMD: 0.5\%, $0.3 \%$ and $0.4 \%$, respectively) (Wong 2014). Age is the main risk factor for AMD across ethnic groups: the late stage ranges from $0.1 \%$ (95\% CrI $0.1 \%$ to $0.2 \%$ ) among individuals aged 50 to 59 years to $3.3 \%$ ( $95 \% \mathrm{CrI} 2.2 \%$ to $4.6 \%$ ) among individuals aged 80 years or more. The global projected cases of AMD in 2040 are 288 million (95\% CrI 205 to 399), with the largest number of cases in Asia and Europe (Wong 2014).

In Western countries, AMD is the leading cause of severe, irreversible blindness in individuals over 50 years of age (Chappelow 2008). The loss of visual perception occurs primarily in the late stages of the disease due to neovascular (i.e., wet) AMD, geographic atrophy, or a combination of the two processes (Lim 2012). In particular, neovascular AMD accounts for $90 \%$ of all cases of severe vision loss due to AMD (Ferris 1984). In neovascular AMD, blood vessels grow aberrantly into the subretinal, intraretinal, and intrachoroidal space. These abnormal vessels can leak serous fluid, including lipid and blood, causing swelling and damage to the macula (Green 1993). Without treatment, its natural course will result in a fibrous scar that greatly diminishes central visual capacity. In the UK, the estimated annual incidence of neovascular AMD is 2.3 (95\% CrI 1.4 to 4.0 ) per 1000 women and 1.4 (95\% CrI 0.8 to 2.4) per 1000 men (Owen 2012).

\section{Description of the intervention}

Vascular endothelial growth factor (VEGF) is an angiogenic cytokine that promotes vascular leakage and growth. Its signalling is over expressed in neovascular AMD as well as in some tumours (e.g., colorectal cancer). Accordingly, VEGF inhibitors such as bevacizumab and ranibizumab have been used to block its pathological angiogenesis. Bevacizumab is approved by drug regulatory authorities for intravenous use as a cancer therapy, whereas ranibizumab is approved for intravitreal use in the treatment of neovascular AMD.

Bevacizumab and ranibizumab derive from the same anti-VEGF mouse monoclonal antibody (Ferrara 2006), but differ in the monoclonal antibody fragment and glycosylation of proteins (Meyer 2011). In intravitreal injections, both drugs can enter systemic circulation, although bevacizumab, a full-length antibody, exhibits a longer half-life (Avery 2014). The shared molecular structure and pharmacological profile of the two drugs, as well as bevacizumab's therapeutic utility (Braithwaite 2014), have led to the widespread off-label/unlicensed use of bevacizumab to treat neovascular AMD as a less expensive alternative to ranibizumab (Miller 2013).

The approved dosage of ranibizumab as indicated in the 'Summary of Product Characteristics' is $0.5 \mathrm{mg}$. After intravitreal administration, ranibizumab slowly moves into systemic circulation (Xu 2013). The dosage of bevacizumab for intravitreal injection most commonly used in published studies is $1.25 \mathrm{mg}$ (Horsley 2009), as it is not toxic to the retina (Manzano 2006) and is approximately 400 -fold less than the intravenous dose used in cancer therapy (Schmucker 2010). In fact, when administered intravitreally, only a small fraction of bevacizumab enters systemic circulation (Kim 2009; Krohne 2008; Stergiou 2011). Retreatment regimens include continuous (i.e., monthly injections) and discontinuous treatment, including 'as needed' (pro re nata (PRN)) and 'treatand-extend' regimens in which the drug is injected less frequently as long as there is no recurrence of neovascular manifestations.

\section{Why it is important to do this review}

The use of bevacizumab or ranibizumab relies on evidence of superiority of one drug over the other. A number of factors will influence the decision, including different profiles for effectiveness, ocular and systemic adverse events, resource use, and the feasibility of the intervention in practice. There is evidence that bevacizumab is associated with a relative improvement in best corrected visual acuity (BCVA) similar to that of ranibizumab (-1.15 letters; $95 \%$ confidence interval (CI) -2.82 to 0.51 ). BCVA outcome favoured a continuous treatment regimen when compared with a discontinuous regimen (-2.23 letters; $95 \%$ CI -3.93 to -0.53 ) (Chakravarthy 2013).

There is limited evidence that bevacizumab might be associated with higher ocular adverse events (risk ratio (RR) 3.1; 95\% CI 1.1 to 8.9); however, the absolute rates of serious ocular adverse events (e.g., endophthalmitis, uveitis) were low $(\leq 2.1 \%)$ (Schmucker 2012), and may not have related to the drugs, but to the procedure (i.e., intravitreal injection). Most discordant judgements between the two drugs focused on differences in their systemic safety.

VEGF has important growth-promoting and maintenance roles in a variety of cells and tissues, raising concern that these agents may interfere with normal physiology and worsen conditions such as coronary or peripheral arterial diseases. VEGF inhibition in cancer patients treated systemically with bevacizumab, at higher dosages than are given in neovascular AMD, was found to increase the risk of fatal events due to haemorrhage (RR 2.77; 95\% CI 1.07 to 7.16 ), neutropenia (RR 2.37; $95 \%$ CI 0.61 to 9.18 ), and gastrointestinal perforation (RR 2.45; 95\% CI 0.63 to 9.51) ( Ranpura 2011). Accordingly, concerns have been expressed about the systemic safety of anti-VEGF drugs, even at the small doses delivered with intravitreal injection (Lim 2011). 
Comparison of AMD Treatments Trials (CATT), the first published large randomised controlled trial (RCT) comparing ranibizumab directly with bevacizumab, reported a statistically significant finding of an excess of serious systemic adverse events (e.g., life-threatening or resulting in significant patient disability) related to bevacizumab when compared with ranibizumab (RR 1.29; 95\% CI 1.01 to 1.66$)$. The publication of results from a second large RCT, Inhibition of VEGF in Age-related Choroidal Neovascularisation (IVAN) (Chakravarthy 2013), and the concomitant meta-analysis of the CATT and IVAN safety results at two years prompted by the IVAN and CATT data monitoring committees, again showed that the sum of all serious adverse events differed by treatment regimen. However, when serious adverse events were compared by organ system class or by specific adverse events (e.g., arterial thrombotic event), there were no differences between the drugs. These results and their varying interpretations fuelled medical and health policy debates on the off-label use of bevacizumab as a far less costly alternative to ranibizumab.

\section{O B J E C T I VES}

To assess the systemic safety of intravitreal bevacizumab (brand name Avastin®; Genentech/Roche) compared with intravitreal ranibizumab (brand name Lucentis ${ }^{\circledR}$; Novartis/Genentech) in people with neovascular AMD.

\section{METHODS}

\section{Criteria for considering studies for this review}

\section{Types of studies}

We included head-to-head randomised controlled trials (RCTs) comparing bevacizumab and ranibizumab in people affected by neovascular age-related macular degeneration (AMD). We included trials irrespective of the dosage, whether treatment is continuous or discontinuous, or duration of follow-up.

\section{Types of participants}

We included people affected by neovascular AMD irrespective of age, sex, or progression of the condition.

\section{Types of interventions}

We compared the systemic safety of intravitreal bevacizumab (brand name Avastin ${ }^{\circledR}$; Genentech/Roche) with ranibizumab (brand name Lucentis ${ }^{\circledR}$; Novartis/Genentech). For this review, we did not consider placebo-controlled trials and trials comparing other anti-vascular endothelial growth factor (VEGF) agents approved for neovascular AMD (e.g., pegaptanib and aflibercept). These studies can contribute to a large network meta-analysis that simultaneously summarises direct evidence (which comes from studies directly randomising treatments of interest) and indirect evidence (which comes from studies comparing treatments of interest with placebo) (Salanti 2008). We will be completing the network meta-analysis as a second phase of this project, to address the issue of the relative effectiveness and safety across a network of RCTs testing anti-VEGF agents.

\section{Types of outcome measures}

\section{Primary outcomes}

Primary outcome domains included:

1. All-cause deaths.

2. All serious systemic adverse events (hereinafter referred to as All SSAEs), the sum of individuals affected by one or more SSAEs recorded in a trial. The International Conference on Harmonisation Good Clinical Practice (ICH GCP) Guideline defines SSAEs as medical occurrences that result in death, are life-threatening, require hospital admission or prolongation of hospital stay, cause persistent or significant disability/incapacity, or are medically important events or reactions (ICH 2014). We accepted the definition of SSAE adopted by the study authors, while recognising that some studies may not have adopted the ICH GCP Guideline.

\section{Secondary outcomes}

Secondary outcome domains included:

1. Myocardial infarction (MI).

2. Stroke.

3. Arteriothrombotic event, defined as any participant who has experienced at least one of the following events: a) myocardial infarction, b) non-haemorrhaging stroke, c) angina, d) ischaemic heart disease, e) thrombosis, or f) death from cardiovascular diseases.

4. Serious haemorrhage as defined by each study, including, but not limited to, cerebral, pulmonary, and gastrointestinal haemorrhage (these are usually defined as a haemorrhage that is associated with anaemia, transfusion, haemostatic intervention, hospitalisation, or fatal bleeding).

5. Serious neutropenia as defined by each study (these are usually defined as neutropenia of grade 3 and 4 associated with sepsis and life-threatening infections) (National Cancer Institute 2003).

6. Gastrointestinal perforation.

7. Serious infection as defined by each study, including, but not limited to, pneumonia, lung abscess, and pyothorax (these 
are usually defined as an infection associated with the use of intravenous antibiotic, hospitalisation, intubation, or death). We excluded ocular infections.

8. Treatment-related drug discontinuation.

9. SSAEs classified according to the Medical Dictionary for Regulatory Activities System Organ Classes (MedDRA SOC) (version 17.0) (ICH 2014), including: benign, malignant, or unspecified neoplasms; cardiovascular disorders; gastrointestinal disorders; general disorders and administration site conditions; infections and infestations; nervous system disorders; and respiratory, thoracic, and mediastinal disorders.

10. Serious adverse events previously associated with drugs affecting the VEGF pathway (i.e., arteriothrombotic events, systemic haemorrhage, congestive heart failure, venous thrombotic events, hypertension, and vascular death).

The SSAEs classified by MedDRA SOC differed from our primary outcome All SSAEs as they explored specific subsets of SSAEs, providing the opportunity to explore the biological plausibility of each.

\section{Search methods for identification of studies}

\section{Electronic searches}

We systematically searched CENTRAL (which contains the Cochrane Eyes and Vision Group Trials Register) (2014, Issue 1), Ovid MEDLINE, (January 1946 to March 2014) and EMBASE (January 1980 to March 2014). We used and updated the search strategy prepared for an update of a Cochrane Review on antiangiogenic drug effectiveness for neovascular AMD (Solomon 2014). RCTs that investigated an anti-VEGF treatment compared to another treatment, sham treatment, or no treatment were eligible. We also searched clinical trial registers, including the metaRegister of Controlled Trials ( $m$ RCT) (www.controlled-trials.com), ClinicalTrials.gov (www.clinicaltrials.gov) and the World Health Organization (WHO) International Clinical Trials Registry Platform (ICTRP) (www.who.int/ictrp/search/en) to identify other ongoing studies or completed studies that have not yet been published. We did not use any date or language restrictions in the electronic searches for trials. We last searched the electronic databases on 27 March 2014. For unpublished RCTs, we searched the Internet for pre-publication study presentations at conferences or meetings and contacted study authors seeking information on safety data.

\section{Searching other resources}

We searched the reference lists of relevant studies to identify additional studies.

\section{Data collection and analysis}

We collected and combined outcomes at the maximum follow-up times reported up to a maximum of two years, since this corresponds to the maximal cumulative drug dosage. We conducted a pre-specified subgroup analysis to explore SSAE outcome at the one-year follow-up.

\section{Selection of studies}

Two investigators (EL and GV) independently screened the titles and abstracts of studies identified through the literature searches and additional sources. We retrieved and independently assessed the full text or unpublished reports using predefined inclusion criteria. We resolved discrepancies through discussion and, when necessary, by consulting an additional investigator (LM).

\section{Data extraction and management}

Two investigators independently extracted data (EL and GV) on study characteristics and entered data into RevMan (RevMan 2014). Three investigators extracted data on our safety primary and secondary outcomes (EL, GV, and KK).

\section{Assessment of risk of bias in included studies}

We assessed the risk of bias in each included study following the criteria outlined in Chapter 8 of the Cochrane Handbook for Systematic Reviews of Interventions (Higgins 2011), which addresses the following key domains: randomisation sequence generation; allocation concealment; masking (blinding) of participants, trial personnel, and outcome assessors; incomplete outcome data; selective outcome reporting (i.e., absence of data or non-publication of deaths and All SSAEs - our primary outcomes); and other sources of bias (e.g., early termination of a trial due to benefits or the failure of authors to disclose the study's source of financial support).

We evaluated additional risk of bias items specific to adverse events using the following items: (1) adverse event definition: if the definition of adverse events was pre-specified in the protocol and collected based on standard criteria or classification system (e.g., MedDRA SOC) and (2) method of adverse event assessment: if the researchers actively monitored for adverse events or simply provided spontaneous reporting of adverse events that arose during the study.

If the information in published study reports or unpublished supporting documents (e.g., meeting abstracts or presentations) was partial or inadequate to assess the risk of bias, we contacted the study authors for clarification. If the authors did not respond, we assessed the risk of bias based on the available information.

\section{Measures of treatment effect}


We used the risk ratio (RR) to estimate the relative safety effect of the two anti-VEGF drugs.

\section{Unit of analysis issues}

Individual participants served as the unit of analysis. Since repeated SSAEs can occur in the same participant, we considered the number of individuals with at least one SSAE, rather than the number of SSAEs. However, the individual-level analysis might decrease the statistical power of meta-analyses.

\section{Dealing with missing data}

In all studies, we carried out the analyses, as far as possible, on an intention-to-treat basis. In other words, we attempted to include all participants randomised to each group in the analyses, irrespective of the treatment received or if the participants completed the study follow-up (Akl 2013). As a second option, we collected data on participants who received at least one dose of study medication: as treated analysis.

If there was a discrepancy between the number randomised and the number analysed in each treatment group, we calculated and reported the percentage lost to follow-up in each group. Where data were inadequate to assess the extent of SSAEs, we contacted the principal investigators of included studies to provide any unreported data. Where it was not possible to obtain information on missing data, we recorded this in the data collection form and reported it in the 'Risk of bias' table. We further discussed the extent to which the missing data could alter the results/conclusions of the review.

\section{Assessment of heterogeneity}

We anticipated that a low number of RCTs in a pairwise comparison (due to either the paucity of studies in the field or incomplete reporting) would prevent the formal assessment of statistical heterogeneity. Nevertheless, we calculated the $\mathrm{Chi}^{2}$ and $\mathrm{I}^{2}$ statistics. For the latter, we also calculated and reported the 95\% CI for primary outcomes. $\mathrm{I}^{2}$ estimates the variability among individual study relative risk estimates that is due to statistical heterogeneity rather than to sampling error (Higgins 2003).

\section{Assessment of reporting biases}

We anticipated that a low number of RCTs in a pairwise comparison would also prevent the formal assessment of publication bias.

\section{Data synthesis}

In this context, we reasoned that there may be true differences across the population of potential studies as they may have enrolled participants at different risk levels for adverse events. For instance, some studies might have included participants at high risk for arteriothrombotic events, while others may have excluded such participants. For this reason, we used a Mantel-Haenszel randomeffects model for meta-analyses, which provides a robust estimate when pooling sparse data (Robins 1986). We did not formally adjust for multiplicity of comparisons, but considered this issue when interpreting the analyses.

\section{'Summary of findings' table}

We summarised the strength of evidence for all-cause deaths, All SSAEs, serious systemic infections, arterial thromboembolic events, myocardial infarctions, and strokes, using the Grading of Recommendations Assessment, Development, and Evaluation (GRADE) methodology (Guyatt 2008). We used an iterative electronic correspondence discussion process to reach consensus on: a) the factors that affect confidence in the estimate of effects, including risk of bias (i.e., design and study limitations), imprecision, indirectness (directness in the GRADE approach includes generalisability and applicability), inconsistency of results (i.e., heterogeneity), magnitude of effect, and issues of residual plausible confounding and $\mathrm{b}$ ) the rating of the evidence. We expected the imprecision of RRs to be a limitation when investigating deaths and adverse events in RCTs, which might be rare, therefore we followed the GRADE guidelines for assessing this quality item (Guyatt 2011a; Guyatt 2011b). We focused on the 95\% CI around the absolute effects, considering a follow-up between one and two years as sufficient. We adopted an absolute minimal difference of $1 \%$ for deaths and 5\% for All SSAEs as clinically relevant. When the $95 \%$ CI included treatment effects above these thresholds and, therefore, did not exclude an absolute detrimental effect appreciably less than $1 \%$ and $5 \%$, we evaluated the precision as insufficient and downgraded the quality of the overall evidence. We adopted these thresholds based on rating imprecision guidelines (Guyatt 2011b), as well as our own judgement. We settled a clinical decision threshold boundary of $1 \%$ absolute difference as we reasoned this difference to be important to both patients and health systems. For example, when the absolute difference in death rates between ranibizumab and bevacizumab was very small (absolute difference of $0.3 \%$ with a $95 \%$ CI ranging from $-0.1 \%$ to $0.7 \%$ ), the results of the meta-analysis excluded an important difference favouring either drug, and we did not downgrade the quality of evidence for deaths. We adopted two different imprecision thresholds, $1 \%$ or $5 \%$, because the differing importance of the outcome deaths or serious adverse events influenced our judgement.

We presented the overall evidence in a 'Summary of findings' table with summary estimates of absolute and relative effects and their quality according to the GRADE methodology (Guyatt 2013). For each outcome, we categorised our confidence in the estimate of effect as one of four levels, ranging from very low to high.

\section{Subgroup analysis and investigation of heterogeneity}

We did not plan a subgroup analysis for this review. 


\section{Sensitivity analysis}

We computed a Mantel-Haenszel RR using a fixed-effect model to investigate any influence of small study effects on the pooled RR, since the random-effects model tends to attribute greater weight to small studies with increasing heterogeneity (Sterne 2011).

We performed a sensitivity analysis, excluding unpublished data, as well as a leave-one-out meta-analysis to assess the independent influence of each study on the summary estimate (Tobias 1999).

\section{R E S U L T S}

\section{Description of studies}

\section{Results of the search}

Figure 1 illustrates the process by which studies were selected for inclusion in our meta-analysis.

Figure I. Study flow diagram for screened, included, and excluded study reports

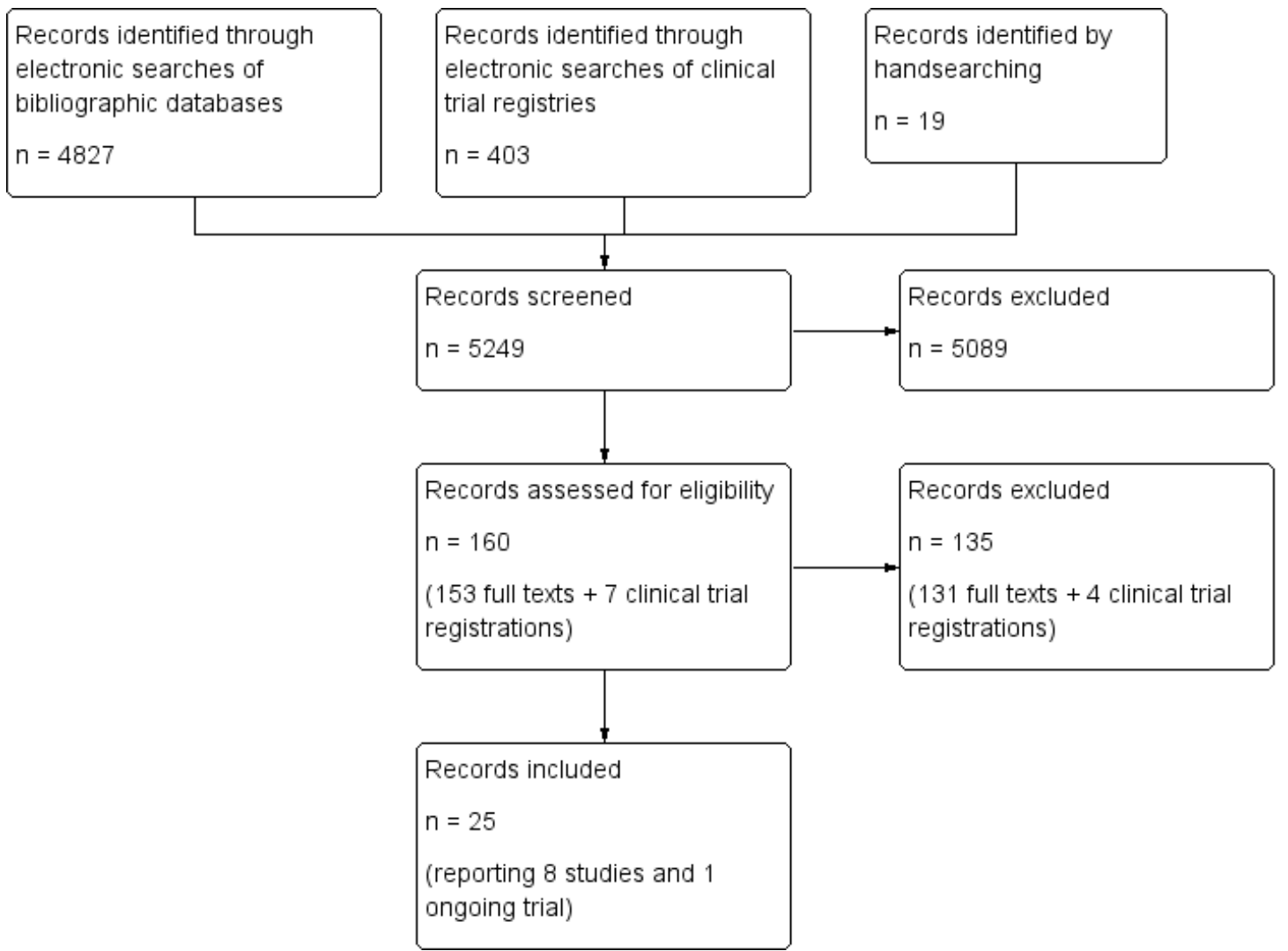

\section{Included studies}

Refer to Characteristics of included studies. Nine RCTs (3665 participants) met our eligibility criteria. Of these, six RCTs were completed and published (Biswas 2011; CATT; GEFAL; IVAN; MANTA; Subramanian 2010), comprising a total of 1362 participants treated with bevacizumab and 1383 treated with ranibizumab. CATT and IVAN provided data at two years (Chakravarthy 2013; Martin 2012), which we used for the primary analyses, whereas the remaining four studies provided data at a maximum follow-up of one year. Three RCTs were unpublished: two were completed (BRAMD; LUCAS), and one is still ongoing (VIBERA). Unpublished data comprised a total of 482 participants treated with bevacizumab and 438 treated with ranibizumab. 
We extracted the unpublished data of LUCAS from a presentation given by the principal investigator at the American Academy of Ophthalmology Retina Subspecialty meeting in 2014. Although this presentation provided information on death and individual cardiovascular SSAE, it did not report the total number of All SSAEs as defined by the study authors. Accordingly, we derived the total number of All SSAEs from the sum of all mutually exclusive SSAEs. We contacted the principal investigator of LUCAS and requested data on this outcome; however, the investigator declined to provide additional information until the trial's publication. We extracted the unpublished data of BRAMD from a presentation at the 2014 meeting of the Association for Research in Vision and Ophthalmology. We contacted the principal investigator of BRAMD to obtain additional data, but did not receive a response. Unpublished data of VIBERA were provided by the study authors. Two studies were conducted in the USA (CATT; Subramanian 2010), six in Europe (BRAMD; GEFAL; IVAN; LUCAS; MANTA; VIBERA), and one in India (Biswas 2011). Studies included populations at variable baseline cardiovascular risks. The proportion of CATT participants who had a prior history of MI, stroke, and transient ischaemic attack at baseline were
$12 \%, 6 \%$, and $6 \%$ of the cases, respectively. These figures were lower in GEFAL (4\%, 4\%, and < 0.5\%) and IVAN (7.5\%, $2.3 \%$, and 5\%), while Biswas 2011, MANTA, and Subramanian 2010 excluded participants with a previous history of vascular thromboembolic events. All trials were non-industry sponsored.

\section{Excluded studies}

We did not exclude any head-to-head RCTs in this review.

\section{Risk of bias in included studies}

The risk of bias for each study is presented in Figure 2. Two unpublished studies were at unclear risk of bias for all items (BRAMD; LUCAS), since limited information was available and the trial authors declined our request for additional information. It is important to note that our 'Risk of bias' assessment differed from that conducted in the parallel efficacy review (Solomon 2014, update under peer review) due to the two reviews' differing outcomes of interest, access to additional information (i.e., from trial authors), and use of additional 'Risk of bias' dimensions related to adverse events. 
Figure 2. 'Risk of bias' summary: review authors' judgements about each risk of bias item for each included study.

\begin{tabular}{|c|c|c|c|c|c|c|c|c|c|}
\hline & 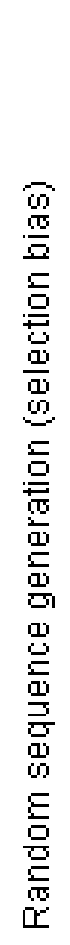 & 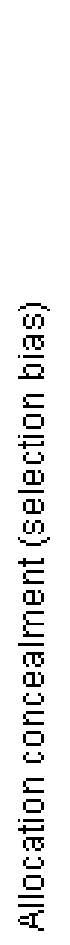 & 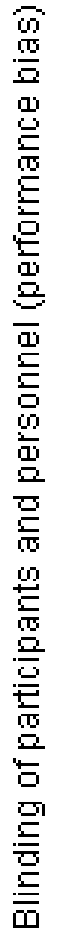 & 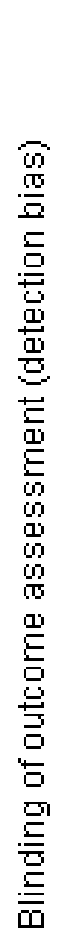 & 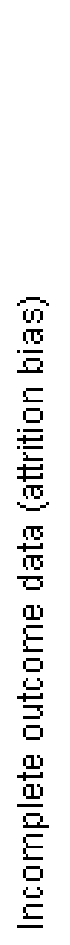 & 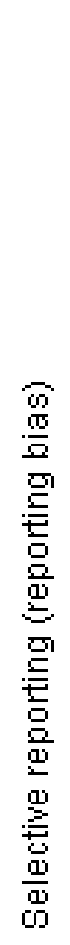 & 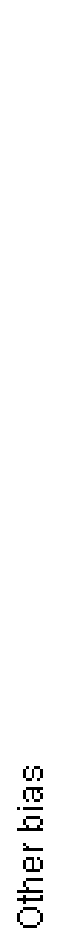 & 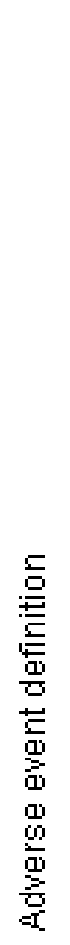 & 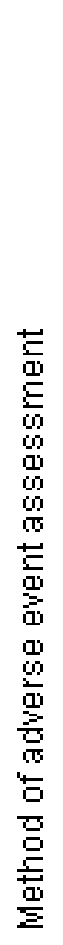 \\
\hline Biswas 2011 & + & $?$ & $?$ & + & $?$ & $?$ & $?$ & $?$ & $?$ \\
\hline BRAMD & $?$ & $?$ & $?$ & $?$ & $?$ & $?$ & $?$ & $?$ & $?$ \\
\hline CATT & + & + & & + & + & $\oplus$ & + & + & + \\
\hline GEFAL & + & + & + & + & + & + & + & + & + \\
\hline IVAN & + & + & + & $?$ & + & ? & + & + & + \\
\hline LUCAS & $?$ & $?$ & $?$ & $?$ & $?$ & $?$ & $?$ & $?$ & $?$ \\
\hline MANTA & + & + & + & + & $?$ & + & + & $?$ & $?$ \\
\hline Subramanian 2010 & $?$ & + & + & + & & $?$ & $?$ & $?$ & $?$ \\
\hline VIBERA & + & + & + & + & + & + & + & + & + \\
\hline
\end{tabular}




\section{Allocation}

The remaining studies generally reported details about random sequence generation and allocation concealment. The treatment allocation was described as masked to clinicians and participants in seven studies (Biswas 2011; CATT; GEFAL; IVAN; MANTA; Subramanian 2010; VIBERA).

\section{Blinding}

In CATT, about half of the participants treated with ranibizumab and a quarter of those treated with bevacizumab may have been aware of their drug assignment through billing reports, while a negligible fraction of participants were unmasked in IVAN. VIBERA used double-masking. In GEFAL and Subramanian 2010, investigators did not report any issue with masking, while we could not find information about the other trials.

\section{Incomplete outcome data}

We contacted study authors for additional information about the risk of bias concerning the missing outcome data: this seemed to be a potential problem across studies since even low rates of missing data, which were between $5 \%$ and $10 \%$ at the end of followup in all studies, could impact estimates for the relative risk of SSAEs. Nevertheless, the opportunity to miss SSAEs during the maintenance period was unlikely to have occurred since all studies, except Biswas 2011, had active SSAE monitoring between injections. Furthermore, missing data were equally distributed between arms, except CATT, in which more information was missing from the bevacizumab arm (35 versus 21 missing data).

\section{Selective reporting}

All studies measured our primary outcomes. Although BRAMD, LUCAS, and VIBERA have not yet been published, we were able to collect data about deaths and All SSAEs.

\section{Other potential sources of bias}

One trial was closed prematurely due to poor recruitment (Subramanian 2010), a common reason for the discontinuation of RCTs (Kasenda 2014). Although empirical evidence demonstrates that discontinued RCTs, on average, overestimate treatment effects, biases are usually associated with RCTs that have been prematurely discontinued for superiority (Bassler 2013). Accordingly, we decided to rate this study at unclear risk of bias.

\section{Risk of bias related to adverse events}

Published studies (CATT; GEFAL; IVAN) and VIBERA appeared to have implemented an appropriate, pre-specified definition of SSAEs and actively monitored them, except for Subramanian 2010 that did not use a pre-specified definition of SSAEs, but actively monitored participants for signs and symptoms possibly related to adverse events, and Biswas 2011 and MANTA, for which both the definition and monitoring of SSAEs were unclear.

\section{Effects of interventions}

See: Summary of findings for the main comparison

\section{Adverse events with bevacizumab compared with ranibizumab}

Figure 3 shows the summary risk ratio (RR) for deaths from eight studies, comprising 3338 participants. The RR of all-cause death for bevacizumab compared with ranibizumab is 1.10 (95\% confidence interval $(\mathrm{CI}) 0.78$ to 1.57 , P value $=0.58)$. Between-study statistical heterogeneity was low $\left(\mathrm{I}^{2}=0 \%\right.$; $95 \%$ CI $0 \%$ to $71 \%$; Analysis 1.1). 
Figure 3. Forest plot of comparison: I Bevacizumab versus ranibizumab, primary analysis at longest followup, outcome: I.I All-cause death.

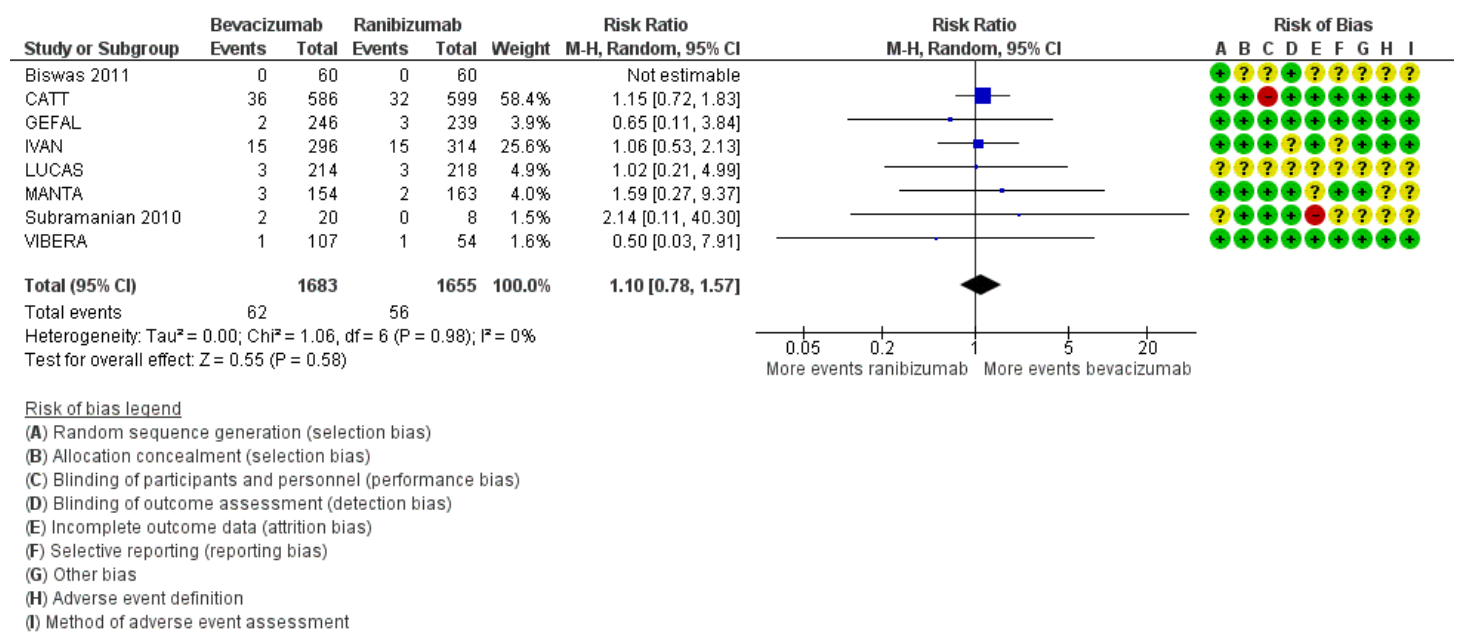

Figure 4 shows the summary RR for All SSAEs (serious systemic adverse events) from nine studies, comprising 3665 participants. The RR of All SSAEs in bevacizumab compared with ranibizumab is 1.08 (95\% CI 0.90 to 1.31 , P value $=0.42$ ) (Analysis 1.2 ). Heterogeneity was moderate $\left(\mathrm{I}^{2}=41 \%\right.$; $95 \%$ CI $0 \%$ to $\left.74 \%\right)$.

Figure 4. Forest plot of comparison: I Bevacizumab versus ranibizumab, longest follow-up, outcome: I.2 All serious systemic adverse events.

\begin{tabular}{|c|c|c|c|c|c|c|c|c|c|}
\hline Study or Subgroup & \multicolumn{2}{|c|}{ Bevacizumab } & \multicolumn{2}{|c|}{ Ranibizumab } & Weight & $\begin{array}{c}\text { Risk Ratio } \\
\text { M-H, Random, } 95 \% \mathrm{Cl}\end{array}$ & \multicolumn{2}{|c|}{$\begin{array}{c}\text { Risk Ratio } \\
\text { M-H, Random, 95\% Cl }\end{array}$} & Risk of Bias \\
\hline BRAMD & 34 & 161 & 37 & 166 & $13.3 \%$ & $0.95[0.63,1.43]$ & & - & ????????? \\
\hline САT & 234 & 586 & 190 & 599 & $29.5 \%$ & $1.26[1.08,1.47]$ & & $\rightarrow-$ & $\odot \odot \odot \odot \odot \odot \odot \odot \odot$ \\
\hline IVAN & 80 & 296 & 81 & 314 & $21.3 \%$ & $1.05[0.80,1.37]$ & & - & $๑ \odot \odot ? \odot ? \odot \odot \odot$ \\
\hline LUCAS & 33 & 214 & 51 & 218 & $14.1 \%$ & $0.66[0.44,0.98]$ & & & ????????? \\
\hline MANTA & 18 & 154 & 15 & 163 & $6.9 \%$ & $1.27[0.66,2.43]$ & & & $๑ \odot \odot \odot ? \odot \odot ? ?$ \\
\hline Subramanian 2010 & 2 & 20 & 0 & 8 & $0.4 \%$ & $2.14[0.11,40.30]$ & & & ?๑๑๑९?? ? ? \\
\hline Total $(95 \% \mathrm{Cl})$ & & 1844 & & 1821 & $100.0 \%$ & $1.08[0.90,1.31]$ & & & \\
\hline \multirow{2}{*}{\multicolumn{7}{|c|}{ 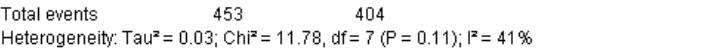 }} & & & \\
\hline \multicolumn{5}{|c|}{$\begin{array}{l}\text { Heterogeneity: } \text { Tau }^{2}=0.03 ; \mathrm{Chi}^{2}=11.78, \mathrm{df}=7(P=0.11) ; \mathrm{I}^{2}=41 \% \\
\text { Test for overall effect: } Z=0.81(P=0.42)\end{array}$} & & & $\begin{array}{ccc} & 1 & 1 \\
0.1 & 0.2 & 0.5 \\
\text { More events ranibizumab }\end{array}$ & $\begin{array}{lccc}1 & 1 & 5 & 10 \\
& 2 & 5 & 10 \\
\text { More events bevacizumab }\end{array}$ & \\
\hline \multicolumn{10}{|c|}{ Risk of bias legend } \\
\hline \multicolumn{10}{|c|}{$\frac{\text { RISk of blas leqend }}{\text { (A) Random sequence generation (selection bias) }}$} \\
\hline \multicolumn{10}{|c|}{ (H) Adverse event definition } \\
\hline (1) Method of adverse & event ass & sment & & & & & & & \\
\hline
\end{tabular}


None of the studies reported serious gastrointestinal perforation, neutropenia, and treatment-related drug discontinuations. We extracted six secondary outcomes from six studies: arterial thromboembolic events, infections, myocardial infarctions, non-ocular haemorrhages, strokes, and vascular events associated with anti-VEGF treatment. We did not find any statistically significant difference in secondary outcomes between bevacizumab and ranibizumab (Analysis 1.3; Analysis 1.4; Analysis 1.5; Analysis 1.6; Analysis 1.7; Analysis 1.8; Analysis 1.9; Analysis 1.10; Analysis 1.11; Analysis 1.12; Analysis 1.14: Analysis 1.15; Analysis 1.16; Analysis 1.17): all comparisons gave estimates with wide CIs with the exception of gastrointestinal disorders MedDRA SOC (RR 1.82; $95 \%$ CI 1.04 to 3.19) (Analysis 1.13).

The main results should be verified once BRAMD, LUCAS, and VIBERA are fully published.

\section{Sensitivity and subgroup analyses}

The one-year random-effects estimates for the relative risk of death $(\mathrm{RR} 1.23$; 95\% CI 0.72 to 2.10 , P value $=0.45)($ Analysis 2.1$)$ and the relative risk of All SSAEs (RR 1.11;95\% CI 0.90 to $1.37, \mathrm{P}$ value $=0.33$ ) (Analysis 2.2) in participants assigned to bevacizumab versus ranibizumab were consistent with estimates derived at the end of the longer follow-up.

Influence (leave-one-out) analyses did not show any influential studies on the RR for death. For All SSAEs, the influence analysis showed that our results were influenced by CATT and LUCAS. The exclusion of CATT moved the overall estimate towards no difference (RR 1.01; 95\% CI 0.82 to 1.25 , P value $=0.92$ ) (Analysis 3.1). The exclusion of LUCAS from the analysis of All SSAEs resulted in a larger RR, with more SSAEs in the bevacizumab group, largely driven by CATT (RR 1.19; 95\% CI 1.06 to 1.34 , P value $=0.004$ ) (Analysis 4.1). The exclusion of all unpublished studies (BRAMD; LUCAS; VIBERA) yielded a RR of 1.12 for death $(95 \%$ CI 0.78 to 1.62 , P value $=0.53)($ Analysis 5.1$)$ and a RR of 1.21 for SSAEs (95\% CI 1.06 to 1.37 , P value $=0.004$ ) (Analysis 5.2 ), indicating a higher risk of SSAEs in those assigned to bevacizumab than ranibizumab. For BRAMD and LUCAS, we were unable to obtain the full definitions of SSAEs and their methods of assessment (see 'Risk of bias' assessment).

Using a fixed-effect meta-analysis model for primary and secondary outcomes on the same studies (Analysis 6.1; Analysis 6.3; Analysis 6.4; Analysis 6.5; Analysis 6.6; Analysis 6.7; Analysis 6.8; Analysis 6.9; Analysis 6.10; Analysis 6.11; Analysis 6.12; Analysis 6.14; Analysis 6.15; Analysis 6.16; Analysis 6.17), we did not find a statistically significant difference between the drugs for deaths. The fixed-effect meta-analysis estimate of All SSAEs was statistically significant (RR 1.12; 95\% CI 1.00 to 1.26 , P value $=0.04)$ (Analysis 6.2). The meta-analysis was dominated by a single study, CATT (weight $=46.9 \%$ ). Again, the estimate of gastrointestinal problems was statistically significant and favoured ranibizumab $($ RR 1.93; 95\% CI 1.20 to 3.11, P value $=0.007)($ Analysis 6.13$)$.

\section{Quality of the evidence}

Refer to Summary of findings for the main comparison for the quality of the evidence for each outcome. In addition to the a priori selected outcomes, we decided also to report the gastrointestinal Medical Dictionary for Regulatory Activities System Organ Classes (MedDRA SOC), the only statistically significant difference we found. Based on the event rates in the studies, the absolute difference between the two drugs is $0.3 \%$ in death rates with a $95 \%$ CI ranging from $-0.8 \%$ to $1.9 \%$, and $1.8 \%$ in All SSAEs rates with a $95 \%$ CI from $-2.2 \%$ to $6.9 \%$. The results of this systematic review do not exclude differences larger than $1 \%$ and $5 \%$ for deaths and All SSAEs that could be important to patients. For gastrointestinal disorders MedDRA SOC, the absolute difference is $1.3 \%$ with more events in the bevacizumab arm and a $95 \%$ CI ranging from $1.3 \%$ to $3.4 \%$. Using GRADE, we assessed the overall quality of the evidence. We began with a GRADE score of four points as the total evidence derives from nine RCTs. We then addressed potential reasons to rate down or up the overall quality of evidence. We downgraded the evidence due to uncertainties in the absolute risks for all outcomes, subtracting one point from the total score for imprecision. We subtracted an additional point for inconsistency as the meta-analysis of All SSAEs was sensitive to the exclusion of CATT or unpublished studies (BRAMD; LUCAS; VIBERA) which, together with the moderate $\mathrm{I}^{2}$ value in the main analysis, we interpreted as evidence of heterogeneity. Thus, the evidence suggests that the true relative safety of bevacizumab versus ranibizumab could be different from that observed in our overall estimates.

The quality of evidence should be reassessed once BRAMD, LUCAS, and VIBERA are fully published.

\section{I SCUSSION}

\section{Summary of main results}

Our systematic review on the systemic safety of bevacizumab directly compared with ranibizumab at one to two years of follow-up in randomised controlled trials (RCTs) on people with neovascular age-related macular degeneration (AMD) did not find conclusive or compelling evidence of an increased or decreased risk of deaths and serious systemic adverse events (SSAEs) - an outcome with qualifying events of death, life-threatening events, hospitalisation, and disability. Although one of our primary outcomes was composite, performed to gain statistical power to show any effects on SSAEs, the $95 \%$ confidence interval (CI) around the pooled estimate of risk ratio (RR) included unity, from 0.90 to 1.31 . With the exception of gastrointestinal disorders, RR estimates for secondary outcomes did not indicate statistically significant differences between bevacizumab and ranibizumab, although the point estimates 
were imprecise. For instance, a few more events were registered in the bevacizumab arm for the cardiac disorder Medical Dictionary for Regulatory Activities System Organ Classes (MedDRA SOC), while a few more events were registered in the ranibizumab arm for myocardial infarction or stroke, with negligible differences. Gastrointestinal disorders were classified according to the MedDRA SOC, which includes abdominal pain, colitis, Crohn's disease, duodenal ulcer, dyspepsia, faecaloma, intestinal obstruction, intestinal perforation, pancreatitis, and vomiting. The rate of these events was low in both groups (1.6\% for ranibizumab and $2.9 \%$ for bevacizumab). The components of this outcome have different clinical importance, with gastrointestinal perforation as one of the most clinically relevant adverse events. Gastrointestinal perforation has been recognised with the systemic use of bevacizumab in patients with cancer (eMC 2014; Hapani 2009), but its incidence was limited $(0.9 \%$; $95 \%$ CI $0.7 \%$ to $1.2 \%)$. It remains unclear whether low-dose ocular administration might or might not cause similar harms. We were unable to collect data on the effect of the drugs on gastrointestinal perforation as well as other outcomes of interest, such as severe neutropenia, since they were reported in only one study.

Initial reports of studies exploring the relative safety of bevacizumab and ranibizumab indicated a possible increased risk for bevacizumab (CATT), with subsequent studies showing no increased risk (Biswas 2011; BRAMD; GEFAL; IVAN; LUCAS; MANTA; Subramanian 2010; VIBERA), therefore the sequence of findings conforms to what has been called the Proteus phenomenon (Ioannidis 2005a). However, diverging from the Proteus phenomenon, in which the first trial is small and opportunistic, in our context the CATT study is a large RCT that is considered a major breakthrough of independent research. Moreover, it is not uncommon for there to be conflicting results between a large RCT and meta-analyses on the same topic (LeLorier 1997). Reasons for these discrepancies include different patient populations: CATT may have included patients with diverse baseline risks for SSAEs compared with other included RCTs. Additionally, a few patients at high risks may greatly influence the overall estimate (Ioannidis 1997): CATT accounts for the majority of the SSAEs of interest recorded in our meta-analysis, as shown in our leave-one-out sensitivity analysis. There are two possible interpretations of these findings. First, the safety of the two drugs is not the same across different levels of patient risk. However, trials that also included patients at high risks did not replicate CATT findings (BRAMD; CATT; GEFAL; IVAN). Second, the two drugs have the same safety for all patients, and the reasons for differences relate to the limitations of individual trials. The CATT investigators carefully considered the potential failure of their masking efforts: through billing documents, patients and health professionals may have known the intervention assigned. This ancillary information might have led to disproportionate and varying levels of attention to SSAEs in patients by health professionals. Our study calls for a more cautious interpretation of the CATT SSAE finding: the result of one trial is not substantially significant and certain enough to activate drug policies (i.e., restricted reimbursement for the off-label use of medicines). Instead, we urge decision-makers to evaluate the result based on its substantive robustness and replicability, components that a community with rational standards for interpreting evidence would agree are necessary for a result to be considered relevant and objective, beyond merely statistically significant, to guide political action (Esarey 2014; Ioannidis 2005b).

Our sensitivity analysis, adopting a fixed-effect, featured a marginal statistically significant difference $(\mathrm{P}$ value $=0.04)$ favouring ranibizumab in terms of SSAEs. However, under the assumption that no between-study heterogeneity existed, the weight of CATT became more prominent. For the above reasons, we qualitatively judged the fixed-effect model assumption to be too strong given the potential differences between the trials in eligibility criteria (inclusion of high-risk patients or not), baseline disease severity of patient populations, follow-up duration (one or two years), and successful masking of participants, investigators, and assessors, with moderate between-study heterogeneity present in the SSAE analysis $\left(\mathrm{I}^{2}=41 \%\right)$.

\section{Overall completeness and applicability of evidence}

Health professionals and decision-makers should consider the following factors when interpreting the results of this review:

1. We found nine non-industry sponsored RCTs. This represents a remarkable amount of investment from researchers and healthcare systems to answer an important clinical question. We are unaware of other examples with such a large number of head-to-head non-industry sponsored RCTs.

2. RCTs were conducted across several countries: Austria, France, Germany, India, the Netherlands, Norway, UK, and the USA.

3. The intervention implemented in these RCTs reflects the doses and regimens that are used in other countries.

4. The included participants represent a wide spectrum of risks for cardiovascular diseases.

This review provides evidence that is complete, comprehensive, and applicable to practice.

\section{Quality of the evidence}

We graded the overall quality of evidence as moderate for the majority of outcomes. Most published RCTs were thoroughly planned and well-executed investigations that reported evidence on adverse events. Allocation concealment, masking, and attrition did not raise major concerns. We could not evaluate the quality for two unpublished studies. Reasons to rate down the overall quality of evidence included large confidence intervals for all safety outcomes (i.e., imprecision) and the sensitivity of the SSAE metaanalysis to a few studies. 
The quality of evidence should be reassessed once the BRAMD and LUCAS trials are fully published.

\section{Potential biases in the review process}

Several important limitations should be noted, mainly due to the rapid timeline of the review. We obtained limited information from study authors, and several studies still had items of unclear risk of bias. The uncertain role of missing data, which is typically reported only in relation to efficacy, but not for safety, may have influenced the overall analyses; the direction of the bias is unpredictable, but we believe that the likelihood of this bias is minimal. The analysis of SSAEs was sensitive to the exclusion of data from an unpublished study, LUCAS. For this study, we derived SSAEs as a sum of all single SSAEs listed, assuming that one SSAE occurred in each participant. We assessed the quality of the evidence based solely on the adverse event profile related to the two drugs (i.e., we did not evaluate the comparative effectiveness of the drugs). A final limitation is that we did not include: i) outcomes such as blood pressure or left ventricular ejection fraction, which are signs that can lead to cardiovascular diseases, or ii) observational comparative studies (Campbell 2012; Curtis 2010; French 2011), since this would have required a different review methodology. Strengths of this review include the extensive search for published and unpublished studies; a multi-disciplinary team; an in-depth assessment of key findings in light of single studies as well the overall evidence; a standard methodology for conducting the review; and an a priori definition of the variables to include in the primary outcome SSAEs.

\section{Agreements and disagreements with other studies or reviews}

Our safety results differed from those of a parallel efficacy review (Solomon 2014, update under peer review), which showed an increased risk of adverse events for bevacizumab compared to ranibizumab (RR 1.27; 95\% CI 1.06 to 1.52 ). Our systematic review included only head-to-head RCTs (the ones that directly compare bevacizumab and ranibizumab), while Solomon 2014 included head-to-head as well standard treatment, placebo, or shamcontrolled RCTs. However, we included three additional RCTs (BRAMD; LUCAS; VIBERA) compared with the Solomon 2014 update, considering both published and unpublished data. Furthermore, we provide a comprehensive assessment of the contribution of each RCT to the meta-analyses, a 'Risk of bias' assessment that specifically targets risks related to adverse events, and several analyses to test the robustness of the overall estimates.

Several previous reviews have assessed the safety of antiangiogenic therapy in people with neovascular AMD. Among these, Cruess 2014 recently reviewed different types of evidence on the safety of intravitreal bevacizumab in a non-systematic fashion, includ- ing results from some RCTs and non-randomised studies, studies on systemic vascular endothelial growth factor (VEGF) levels after intravitreal injections, and differences in molecular structure of bevacizumab and ranibizumab. However, bevacizumab was not directly compared with ranibizumab. Chakravarthy 2012 compared serum VEGF levels achieved with bevacizumab and ranibizumab: these were lower with bevacizumab, although the change in this biomarker has yet to be associated with adverse outcomes. Chakravarthy 2013 pooled data from IVAN and CATT at two years and could not show a difference between bevacizumab and ranibizumab for deaths and arterial thrombotic events, but pointed out that SSAEs were higher with bevacizumab because of the enduring influence of the larger CATT study. In a recent systematic review and meta-analysis, Thulliez 2014 showed no differences between bevacizumab and ranibizumab for the risk of major cardiovascular events or non-ocular haemorrhagic events in people with neovascular AMD, but found significantly increased venous thromboembolic events for bevacizumab by cumulating data from three unspecified RCTs directly comparing bevacizumab with ranibizumab, totalling 15 events (12 in the bevacizumab arm versus three in the ranibizumab arm). We aggregated thromboembolic events with other serious adverse events previously associated with drugs affecting the VEGF pathway (e.g., arteriothrombotic events, vascular events, and death), totalling 150 events (79 in the bevacizumab arm versus 71 in the ranibizumab arm, Analysis 1.11), and did not find significant differences. A systematic review by Zhang et al analysed both evidence from observational and experimental studies, comparing bevacizumab with ranibizumab for efficacy and safety (Zhang 2014). Again, the authors found only four RCTs (Biswas 2011; CATT; IVAN; Subramanian 2010), interpreted the unadjusted evidence from observational studies as compelling and free of bias, and overstated the strength of causal inference in the conclusions. Finally, another systematic review showed an increased risk of SSAEs in RCTs on several biologics for any indication (Singh 2009).

\section{AUTHORS' CONCLUSIONS}

\section{Implications for practice}

From the evidence presented in nine randomised controlled trials (RCTs) comprising 3665 participants with neovascular age-related macular degeneration (AMD), we did not observe evidence of a difference in the relative safety between intravitreal bevacizumab and ranibizumab for deaths, All SSAEs (serious systemic adverse events), or specific subsets of SSAEs in the first one to two years of treatment, with the exception of gastrointestinal disorders Medical Dictionary for Regulatory Activities System Organ Classes (MedDRA SOC). With regard to available data on systemic safety, this review provides no significant evidence to support the preferen- 
tial use of either bevacizumab or ranibizumab in the treatment of neovascular AMD.

In absolute terms, if 1000 people were treated with ranibizumab for one or two years, 34 would die and 222 would experience one or more SSAEs. If 1000 people were treated with bevacizumab for one or two years, then about 37 would die and 240 would experience one or more SSAEs. Overall, these differences are small and consistent with chance variation.

We cannot exclude: i) differences larger than $1 \%$ and $5 \%$ for deaths and All SSAEs that could be important to patients and ii) potential differences in safety across the levels of patient risk. As elderly individuals have a variable risk of SSAEs due to multi-morbidity and polypharmacy, the use of bevacizumab and ranibizumab should be closely monitored by physicians. Health professionals should recognise the following patient risk factors before exposing patients to bevacizumab: hypertension, left ventricular dysfunction, haemorrhagic events, inherited or acquired coagulopathy, proteinuria, major surgery, and co-treatment with other drugs that may potentiate the cardiotoxic effects (e.g., anthracyclines) (Cortes 2012; Saif 2006; Wu 2010). These individual risk factors should be carefully considered, particularly in patients with multiple risk factors (e.g., coagulopathy, nephrotic disease, and heart failure).

\section{Implications for research}

The proportion of randomised evidence that has not yet been published is limited (about 25\%) (BRAMD; LUCAS; VIBERA), but highly relevant, and deserves timely public dissemination. Trialists and entities that financially support these trials should consider and encourage quick dissemination of data, pursuing options such as fast-track publication (Manzoli 2014).

We do not support the idea of starting new head-to-head RCTs. Rather, an individual patient data meta-analysis might better assess the exact magnitude of the difference by exploring effect- modifiers such as drug regimen and susceptible patient subgroups, such as those at higher cardiovascular risk. The investigators for the CATT study have made their trial data publicly available and other trial investigators are urged to do the same. Although RCTs are the best tool to investigate both the efficacy and safety of interventions, they continue to show limitations related to the poor reporting of adverse events, as well as their insufficient power to detect the majority of adverse events, even for common adverse events. Complementary information on the occurrence of adverse events may come from observational studies. However, in this setting, observational studies also show limitations. In addition to the concern about potential biases usually associated with observational designs, we anticipate that the large difference in terms of costs between bevacizumab and ranibizumab could affect patient case-mix, thereby challenging the comparability between groups (Bosco 2010). For the investigation of safety data, pharmacovigilance remains necessary to explore these issues in the general population, which often comprises of potentially more susceptible patients.

We believe that our synthesis is a timely and complete summary of the available evidence from RCTs on the relative safety of bevacizumab compared with ranibizumab. We will update the results of this Cochrane Review once new findings are published.

\section{ACKNOW LEDGEMENTS}

The authors would like to thank Anupa Shah, Richard Wormald, and the Cochrane Eyes and Vision Group, who executed the searches and supported the review editorial process. We also thank Sheila Bird, Hugh McIntyre, Tasanee Braithwaite, and Jenny Evans for their comments on the review, as well as Toby Lasserson and Orla Ni Ogain (from the Cochrane Editorial Unit). Finally, the authors thank Laure Huot and Evelyne Decullier of the GEFAL team for their valuable support with the review.

\section{REFEREN CES}

\section{References to studies included in this review}

Biswas 2011 \{published data only\}

Biswas P, Sengupta S, Choudhary R, Home S, Paul A, Sinha $S$. Comparative role of intravitreal ranibizumab versus bevacizumab in choroidal neovascular membrane in age-related macular degeneration. Indian Journal of Ophthalmology 2011;59(3):191-6.

BRAMD \{unpublished data only\}

Schauwvlieghe A-SM, Dijkman G, Hooymans JM, Verbraak FD, Dijkgraaf MG, Peto T, et al.Comparing the effectiveness of bevacizumab to ranibizumab in patients with exudative age-related macular degeneration. BRAMD.
Investigative Ophthalmology and Visual Science 2014;55: ARVO E-Abstract 870.

CATT \{published data only\}

Martin DF, Maguire MG, Fine SL, Ying GS, Jaffe GJ, Grunwald JE, et al.Ranibizumab and bevacizumab for treatment of neovascular age-related macular degeneration: two-year results. Ophthalmology 2012;119(7):1388-98. Martin DF, Maguire MG, Ying GS, Grunwald JE, Fine SL, Jaffe GJ. Ranibizumab and bevacizumab for neovascular age-related macular degeneration. New England Journal of Medicine 2011;364(20):1897-908.

GEFAL \{published data only\}

Kodjikian L, Souied EH, Mimoun G, Mauget-Faysse 
M, Behar-Cohen F, Decullier E, et al.Ranibizumab versus bevacizumab for neovascular age-related macular degeneration: results from the GEFAL noninferiority randomized trial. Ophthalmology 2013;120(11):2300-9.

IVAN \{published data only\}

Chakravarthy U, Harding SP, Rogers CA, Downes SM, Lotery AJ, Culliford LA, et al.Alternative treatments to inhibit VEGF in age-related choroidal neovascularisation: 2-year findings of the IVAN randomised controlled trial. Lancet 2013;382(9900):1258-67.

Chakravarthy U, Harding SP, Rogers CA, Downes SM, Lotery AJ, Wordsworth $S$, et al.Ranibizumab versus bevacizumab to treat neovascular age-related macular degeneration: one-year findings from the IVAN randomized trial. Ophthalmology 2012;119(7):1399-411.

LUCAS \{unpublished data only\} Berg K. Lucentis compared to Avastin Study (LUCAS). AAO Annual Meeting, 2013 subspecialty day. conferencecast.com/AAO/common/sessions.aspx/4/7 (accessed 30 April 2014).

MANTA \{published data only\}

Krebs I, Schmetterer L, Boltz A, Told R, Vecsei-Marlovits V, Egger $S$, et al.A randomised double-masked trial comparing the visual outcome after treatment with ranibizumab or bevacizumab in patients with neovascular age-related macular degeneration. British Journal of Ophthalmology 2013;97(3):266-71.

Subramanian 2010 \{published data only\} Subramanian ML, Abedi G, Ness S, Ahmed E, Fenberg M, Daly MK, et al.Bevacizumab vs ranibizumab for age-related macular degeneration: 1-year outcomes of a prospective, double-masked randomised clinical trial. Eye 2010;24(11): 1708-15.

VIBERA \{unpublished data only\}

NCT00559715. Prevention of vision loss in patients with age-related neovascular macular degeneration by intravitreal injection of bevacizumab and ranibizumab in a typical outpatient setting. clinicaltrials.gov/show/NCT00559715 (accessed 15 August 2014).

\section{Additional references}

\section{Akl 2013}

Akl EA, Johnston BC, Alonso-Coello P, Neumann I, Ebrahim S, Briel M, et al.Addressing dichotomous data for participants excluded from trial analysis: a guide for systematic reviewers. PLoS One 2013;8(2):e57132.

Avery 2014

Avery RL, Castellarin AA, Steinle NC, Dhoot DS, Pieramici DJ, See R, et al.Systemic pharmacokinetics following intravitreal injections of ranibizumab, bevacizumab or aflibercept in patients with neovascular AMD. British Journal of Ophthalmology 2014 July 7 [Epub ahead of print].

Bassler 2013

Bassler D, Montori VM, Briel M, Glasziou P, Walter SD, Ramsay T, et al.Reflections on meta-analyses involving trials stopped early for benefit: is there a problem and if so, what is it?. Statistical Methods in Medical Research 2013;22(2): $159-68$.

\section{Bosco 2010}

Bosco JL, Silliman RA, Thwin SS, Geiger AM, Buist DS, Prout MN, et al.A most stubborn bias: no adjustment method fully resolves confounding by indication in observational studies. Journal of Clinical Epidemiology 2010; 63(1):64-74.

\section{Braithwaite 2014}

Braithwaite T, Nanji AA, Lindsley K, Greenberg PB. Antivascular endothelial growth factor for macular oedema secondary to central retinal vein occlusion. Cochrane Database of Systematic Reviews 2014, Issue 5. [DOI: 10.1002/14651858.CD007325.pub3]

\section{Campbell 2012}

Campbell RJ, Gill SS, Bronskill SE, Paterson JM, Whitehead M, Bell CM. Adverse events with intravitreal injection of vascular endothelial growth factor inhibitors: nested case-control study. BMJ 2012;345:e4203.

\section{Chakravarthy 2012}

Chakravarthy U, Harding SP, Rogers CA, Downes SM, Lotery AJ, Wordsworth $S$, et al.Ranibizumab versus bevacizumab to treat neovascular age-related macular degeneration: one-year findings from the IVAN randomized trial. Ophthalmology 2012;119(7):1399-411.

\section{Chakravarthy 2013}

Chakravarthy U, Harding SP, Rogers CA, Downes SM, Lotery AJ, Culliford LA, et al.Alternative treatments to inhibit VEGF in age-related choroidal neovascularisation: 2-year findings of the IVAN randomised controlled trial. Lancet 2013;382(9900):1258-67.

\section{Chappelow 2008}

Chappelow AV, Kaiser PK. Neovascular age-related macular degeneration: potential therapies. Drugs 2008;68(8): 1029-36.

\section{Cortes 2012}

Cortes J, Calvo V, Ramirez-Merino N, O'Shaughnessy J, Brufsky A, Robert N, et al.Adverse events risk associated with bevacizumab addition to breast cancer chemotherapy: a meta-analysis. Annals of Oncology 2012;23(5):1130-7.

\section{Cruess 2014}

Cruess AF, Giacomantonio N. Cardiac issues of noncardiac drugs: the rising story of avastin in age-related macular degeneration. Ophthalmologica 2014;231(2):75-9.

\section{Curtis 2010}

Curtis LH, Hammill BG, Schulman KA, Cousins SW. Risks of mortality, myocardial infarction, bleeding, and stroke associated with therapies for age-related macular degeneration. Archives of Ophthalmology 2010;128(10): 1273-9.

\section{eMC 2014}

electronic Medicines Compendium (eMC). Avastin $25 \mathrm{mg} / \mathrm{ml}$ concentrate for solution for infusion. www.medicines.org.uk/emc/medicine/15748 (accessed 31 July 2014). 
Esarey 2014

Esarey J, Danneman N. A quantitative method for substantive robustness assessment. jee3.web.rice.edu/ riskstats.pdf (accessed 20 August 2014).

Ferrara 2006

Ferrara N, Damico L, Shams N, Lowman H, Kim R. Development of ranibizumab, an anti-vascular endothelial growth factor antigen binding fragment, as therapy for neovascular age-related macular degeneration. Retina 2006; 26(8):859-70.

\section{Ferris 1984}

Ferris FL, Fine SL, Hyman L. Age-related macular degeneration and blindness due to neovascular maculopathy. Archives of Ophthalmology 1984;102(11):1640-42.

\section{French 2011}

French DD, Margo CE. Age-related macular degeneration, anti-vascular endothelial growth factor agents, and shortterm mortality: a postmarketing medication safety and surveillance study. Retina 2011;31(6):1036-42.

\section{Green 1993}

Green WR, Enger C. Age-related macular degeneration histopathologic studies. The 1992 Lorenz E. Zimmerman Lecture. Ophthalmology 1993;100(10):1519-35.

\section{Guyatt 2008}

Guyatt GH, Oxman AD, Vist GE, Kunz R, Falck-Ytter Y, Alonso-Coello P, et al.GRADE: an emerging consensus on rating quality of evidence and strength of recommendations. BMJ 2008;336(7650):924-6.

\section{Guyatt 2011a}

Guyatt G, Oxman AD, Akl EA, Kunz R, Vist G, Brozek $\mathrm{J}$, et al.GRADE guidelines: 1. Introduction-GRADE evidence profiles and summary of findings tables. Journal of Clinical Epidemiology 2011;64(4):383-94.

\section{Guyatt 2011b}

Guyatt GH, Oxman AD, Kunz R, Brozek J, Alonso-Coello P, Rind D, et al.GRADE guidelines 6 . Rating the quality of evidence-imprecision. Journal of Clinical Epidemiology 2011;64(12):1283-93.

\section{Guyatt 2013}

Guyatt GH, Oxman AD, Santesso N, Helfand M, Vist G, Kunz R, et al.GRADE guidelines: 12. Preparing summary of findings tables-binary outcomes. Journal of Clinical Epidemiology 2013;66(2):158-72.

Hapani 2009

Hapani S, Chu D, Wu S. Risk of gastrointestinal perforation in patients with cancer treated with bevacizumab: a metaanalysis. The Lancet. Oncology. 2009;10(6):559-68.

\section{Higgins 2003}

Higgins JP, Thompson SG, Deeks JJ, Altman DG. Measuring inconsistency in meta-analyses. BMJ 2003;327 (7414):557-60.

\section{Higgins 2011}

Higgins JPT, Altman DG, Sterne JAC (editors). Chapter 8: Assessing risk of bias in included studies. In: Higgins JPT, Green S (editors). Cochrane Handbook for Systematic
Reviews of Interventions Version 5.1.0 (updated March 2011). The Cochrane Collaboration, 2011. Available at www.cochrane-handbook.org.

Horsley 2009

Horsley W. NHS North East Treatment Advisory Group. Bevacizumab (Avastin $®$ ) in the management of neovascular age-related macular degeneration. www.netag.nhs.uk/files/appraisalreports/Bevacizumab\%20-Avastin-\%20AMD\%20\%20NETAG\%20appraisal\%20report\%20July\%202011.pdf (accessed 15 August 2014).

\section{ICH 2014}

International Conference on Harmonisation of Technical Requirements for Registration of Pharmaceuticals for Human Use (ICH). Introductory guide MedDRA version 17.0. www.meddra.org/sites/default/files/guidance/file/ intguide $17^{\circ} 0^{\circ}$ english.pdf (accessed 13 July 2014).

\section{Ioannidis 1997}

Ioannidis JP, Lau J. The impact of high-risk patients on the results of clinical trials. Journal of Clinical Epidemiology 1997;50(10):1089-98.

\section{Ioannidis 2005a}

Ioannidis JP, Trikalinos TA. Early extreme contradictory estimates may appear in published research: the Proteus phenomenon in molecular genetics research and randomized trials. Journal of Clinical Epidemiology 2005;58(6):543-9.

\section{Ioannidis 2005b}

Ioannidis JP. Contradicted and initially stronger effects in highly cited clinical research. JAMA 2005;294(2):218-28.

\section{Kasenda 2014}

Kasenda B, von Elm E, You J, Blumle A, Tomonaga Y, Saccilotto R, et al.Prevalence, characteristics, and publication of discontinued randomized trials. JAMA 2014; $311(10): 1045-51$.

Kim 2009

Kim H, Robinson SB, Csaky KG. FcRn receptor-mediated pharmacokinetics of therapeutic IgG in the eye. Molecular Vision 2009;15:2803-12.

\section{Krohne 2008}

Krohne TU, Eter N, Holz FG, Meyer CH. Intraocular pharmacokinetics of bevacizumab after a single intravitreal injection in humans. American Journal of Ophthalmology 2008;146(4):508-12.

\section{LeLorier 1997}

LeLorier J, Grégoire G, Benhaddad A, Lapierre J, Derderian F. Discrepancies between meta-analyses and subsequent large randomized, controlled trials. New England Journal of Medicine 1997;337(8):536-42.

$\operatorname{Lim} 2011$

Lim LS, Cheung CM, Mitchell P, Wong TY. Emerging evidence concerning systemic safety of anti-VEGF agentsshould ophthalmologists be concerned?. American Journal of Ophthalmology 2011;152(3):329-31. 
Lim 2012

Lim LS, Mitchell P, Seddon JM, Holz FG, Wong TY. Agerelated macular degeneration. Lancet 2012;379(9827): $5-11$.

Manzano 2006

Manzano RP, Peyman GA, Khan P, Kivilcim M. Testing intravitreal toxicity of bevacizumab (Avastin). Retina 2006; 26(3):257-61

\section{Manzoli 2014}

Manzoli L, Flacco ME, D’Addario M, Capasso L, De Vito C, Marzuillo C, et al.Non-publication and delayed publication of randomized trials on vaccines: survey. $B M J$ 2014;348:g3058.

Martin 2012

Martin DF, Maguire MG, Fine SL, Ying GS, Jaffe GJ, Grunwald JE, et al.Ranibizumab and bevacizumab for treatment of neovascular age-related macular degeneration: two-year results. Ophthalmology 2012;119(7):1388-98.

Meyer 2011

Meyer CH, Holz FG. Preclinical aspects of anti-VEGF agents for the treatment of wet AMD: ranibizumab and bevacizumab. Eye 2011;25(6):661-72.

Miller 2013

Miller JW, Le Couter J, Strauss EC, Ferrara N. Vascular endothelial growth factor a in intraocular vascular disease. Ophthalmology 2013;120(1):106-14.

National Cancer Institute 2003

National Cancer Institute. Cancer Therapeutics Evaluation Program. Common Toxicity Criteria for Adverse Events. 3rd Edition. Bethesda, MD: National Cancer Institute, 2003.

\section{Owen 2012}

Owen CG, Jarrar Z, Wormald R, Cook DG, Fletcher AE Rudnicka AR. The estimated prevalence and incidence of late stage age related macular degeneration in the UK. British Journal of Ophthalmology 2012;96(5):752-6.

\section{Ranpura 2011}

Ranpura V, Hapani S, Wu S. Treatment-related mortality with bevacizumab in cancer patients: a meta-analysis. JAMA 2011;305(5):487-94

\section{RevMan 2014}

The Nordic Cochrane Centre, The Cochrane Collaboration. Review Manager (RevMan). 5.3. Copenhagen: The Nordic Cochrane Centre, The Cochrane Collaboration, 2014.

\section{Robins 1986}

Robins J, Breslow N, Greenland S. Estimators of the Mantel-Haenszel variance consistent in both sparse data and large-strata limiting models. Biometrics 1986;42(2):311-23.

Saif 2006

Saif MW, Mehra R. Incidence and management of bevacizumab-related toxicities in colorectal cancer. Expert Opinion on Drug Safety 2006;5(4):553-66.

\section{Salanti 2008}

Salanti G, Higgins JP, Ades AE, Ioannidis JP. Evaluation of networks of randomized trials. Statistical Methods in Medical Research 2008;17(3):279-301.
Schmucker 2010

Schmucker C, Ehlken C, Hansen LL, Gerd A, Agostini HT, Lelgemann M. Intravitreal bevacizumab (Avastin) vs. ranibizumab (Lucentis) for the treatment of age-related macular degeneration: a systematic review. Current Opinion in Ophthalmology 2010;21(3):218-26.

\section{Schmucker 2012}

Schmucker C, Ehlken C, Agostini HT, Antes G, Ruecker $\mathrm{G}$, Lelgemann $\mathrm{M}$, et al.A safety review and metaanalyses of bevacizumab and ranibizumab: off-label versus goldstandard. PLoS One 2012;7:e42701.

\section{Singh 2009}

Singh JA, Christensen R, Wells GA, Suarez-Almazor ME, Buchbinder R, Lopez-Olivo MA, et al.A network metaanalysis of randomized controlled trials of biologics for rheumatoid arthritis: a Cochrane overview. CMAJ 2009; 181(11):787-96.

\section{Solomon 2014}

Solomon SD, Lindsley K, Vedula SS, Krzystolik MG, Hawkins BS. Anti-vascular endothelial growth factor for neovascular age-related macular degeneration. Cochrane Database of Systematic Reviews 2014, Issue 8. [DOI: 10.1002/14651858.CD005139.pub3]

\section{Stergiou 2011}

Stergiou PK, Symeonidis C, Dimitrakos SA. Descending doses of intravitreal bevacizumab for the regression of diabetic neovascularization. Acta Ophthalmologica 2011;89 (3):218-21.

\section{Sterne 2011}

Sterne JAC, Egger M, Moher D (editors). Chapter 10: Addressing reporting biases. In: Higgins JPT, Green $S$ (editors). Cochrane Handbook for Systematic Reviews of Intervention. Version 5.1.0 (updated March 2011). The Cochrane Collaboration, 2011. Available at www.cochranehandbook.org.

Thulliez 2014

Thulliez M, Angoulvant D, Le Lez ML, Jonville-Bera AP, Pisella PJ, Gueyffier F, et al.Cardiovascular events and bleeding risk associated with intravitreal antivascular endothelial growth factor monocolonal antibodies: a systematic review and meta-analysis. JAMA Ophthalmology 2014 July 24 [Epub ahead of print].

\section{Tobias 1999}

Tobias A. Assessing the influence of a single study in the meta-analysis estimate. Stata Technical Bulletin 1999;8: $1-48$.

\section{Wong 2014}

Wong WL, Su X, Li X, Cheung CMG, Klein R, Cheng CY, et al.Global prevalence of age-related macula degeneration and disease burden projection for 2020 and 2040: a systematic review and meta-analysis. Lancet Global Health 2014;2(2):e106-16. 
Wu 2010

Wu S, Kim C, Baer L, Zhu X. Bevacizumab increases risk for severe proteinuria in cancer patients. Journal of the American Society of Nephrology 2010;21(8):1381-9.

Xu 2013

Xu L, Lu T, Tuomi L, Jumbe N, Lu J, Eppler S, et al.Pharmacokinetics of ranibizumab in patients with neovascular age-related macular degeneration: a population approach. Investigative Ophthalmology and Visual Science 2013;54(3):1616-24.

\section{Zhang 2014}

Zhang XY, Guo XF, Zhang SD, He JN, Sun CY, Zou Y, et
al.Comparison of bevacizumab and ranibizumab in agerelated macular degeneration: a systematic review and metaanalysis. International Journal of Ophthalmology 2014;7(2): 355-64.

\section{References to other published versions of this review}

\section{Moja 2014}

Moja L, Lucenteforte E, Kwag KH, Bertele V, Campomori A, Chakravarthy U, et al.Systemic safety of bevacizumab versus ranibizumab for neovascular age-related macular degeneration. Cochrane Database of Systematic Reviews 2014, Issue 7. [DOI: 10.1002/14651858.CD011230]

* Indicates the major publication for the study 


\section{CHARACTERISTICS OF STUDIES}

\section{Characteristics of included studies [ordered by study ID]}

\section{Biswas 2011}

Methods

Number randomised (total and per group): 120 participants randomly assigned to study treatment; 60 in bevacizumab group and 60 in ranibizumab group

Exclusions after randomisation: none

Number analysed (total and per group): 104 total participants; 50 in bevacizumab group and 54 in ranibizumab group

Unit of analysis: individuals ( 1 study eye per participant)

Losses to follow-up: 16 participants: reasons for losses to follow-up not reported (10 in bevacizumab group, 6 in ranibizumab group)

Compliance: $104 / 120$ participants completed the study

Intention-to-treat analysis: no, 16 participants enrolled and randomised were not included in analysis

Reported power calculation: “... aimed to enrol a total of 120 patients ... this number was arrived at by the investigators after considering the sample size of the available literature". However, the number of enrolled participants seem not adequate to detect differences in adverse events

Unusual study design: randomisation logistics were complicated (see 'Risk of bias' table below)

Participants

Country: 2 study centres in Kolkata, India

Age: not reported for 120 enrolled participants (mean 64.4 years in analysed bevacizumab group; mean 63.5 years in analysed ranibizumab group)

Gender (per cent): not reported for 120 enrolled participants (22/54 (41\%) men and $32 / 54$ (59\%) women in analysed bevacizumab group; $28 / 50(56 \%)$ men and 22/50 (44\%) woman for analysed ranibizumab group)

Inclusion criteria: age 50 or older; presence of subfoveal or juxta foveal CNV of any type; active leakage pattern; baseline BCVA between 35 to 70 ETDRS letters; baseline central macular thickness greater than or equal to $250 \mu \mathrm{m}$, measured by OCT

Exclusion criteria: previous treatment for $\mathrm{CNV}$ in either eye; macular scarring; any coexisting ocular disease or pathology; monocular participants; history of ocular surgery within 6 months of enrolment; history of cerebrovascular accident and myocardial infarction

Equivalence of baseline characteristics: gender imbalance between analysed groups Diagnoses in participants: all with subfoveal or juxta foveal CNV; 24/54 participants with occult CNV in ranibizumab group and 22/50 participants with occult CNV in bevacizumab group

History of cardiovascular events at baseline (bevacizumab, ranibizumab): participants with MI, stroke, TIA were excluded

Intervention 1: $1.25 \mathrm{mg}$ intravitreal bevacizumab every month for first 3 months; retreatment afterwards based on OCT or VA changes

Intervention 2: $0.5 \mathrm{mg}$ intravitreal ranibizumab every month for first 3 months; retreatment afterwards based on OCT or VA changes

Length of follow-up:

Planned: 18 months 
Biswas 2011 (Continued)

Actual: 18 months

Outcomes

Primary outcomes, as defined: "changes in BCVA and CMT from baseline (month 0) to month 18 "

Secondary outcomes, as reported: blood pressure measurements; reports of unusual extremity pain

Adverse events: classification method definition not reported

Intervals at which outcome assessed: monthly through 18 months

Type of study: published
Funding sources: reported “nil”
Declarations of interest: "none declared”
Study period: April 2007 to April 2009
Reported subgroup analyses: yes, for participants with predominantly classic CNV

Risk of bias

\begin{tabular}{|c|c|c|}
\hline Bias & Authors' judgement & Support for judgement \\
\hline $\begin{array}{l}\text { Random sequence generation (selection } \\
\text { bias) }\end{array}$ & Low risk & $\begin{array}{l}\text { "Using random numbers tables, } 60 \text { num- } \\
\text { bers were randomly picked up from } 1 \text { to } \\
120 \text { and assigned to group A while the re- } \\
\text { maining sixty numbers were assigned to } \\
\text { group B." }\end{array}$ \\
\hline Allocation concealment (selection bias) & Unclear risk & $\begin{array}{l}\text { "... randomization of the } 120 \text { numbers into } \\
\text { two groups was done before initiation of } \\
\text { enrolment itself. Upon initiation of enrol- } \\
\text { ment, the patients were numbered sequen- } \\
\text { tially based on the serial order of enrolment } \\
\text { in the study. Depending on the enrolment } \\
\text { number, the patients were automatically as- } \\
\text { signed to either group A or B based on the } \\
\text { prior randomization of number 1-120 into } \\
\text { two equal groups using random number ta- } \\
\text { bles." }\end{array}$ \\
\hline
\end{tabular}

Blinding of participants and personnel Unclear risk

Masking of participants not reported (performance bias)

All outcomes

Blinding of outcome assessment (detection Low risk bias)

All outcomes

Incomplete outcome data (attrition bias) Unclear risk All outcomes

"All assessors were masked to the group of patient they were following up."

$16(13 \%)$ participants lost to follow-up were excluded from the analyses; 6 in the ranibizumab group and 10 in the bevacizumab group 
Biswas 2011 (Continued)

\begin{tabular}{|c|c|c|}
\hline Selective reporting (reporting bias) & Unclear risk & $\begin{array}{l}\text { Reported outcome: adverse events. Deaths } \\
\text { were not mentioned but it is unlikely that } \\
\text { any occurred }\end{array}$ \\
\hline Other bias & Unclear risk & $\begin{array}{l}\text { No protocol or clinical trial registration was } \\
\text { identified for this study. Outcomes were re- } \\
\text { ported for stated outcomes in the methods } \\
\text { section of the published report; however, } \\
\text { only P values were reported for between- } \\
\text { group comparisons and no standard devia- } \\
\text { tion or variance measures were reported for } \\
\text { continuous outcomes }\end{array}$ \\
\hline Adverse event definition & Unclear risk & Not reported \\
\hline Method of adverse event assessment & Unclear risk & Not reported \\
\hline
\end{tabular}

\section{BRAMD}

Number randomised (total and per group): total 327 partic
Exclusions after randomisation: not known
Number analysed (total and per group): 161 to $1.25 \mathrm{mg}$
$0.5 \mathrm{mg}$ ranibizumab group
Unit of analysis: individuals (1 study eye per participant)
Losses to follow-up: not available
Compliance: not available
Intention-to-treat analysis: not known
Reported power calculation: not known
Unusual study design: no

Participants

Country: USA

Age: not known

Gender (per cent): not known

Inclusion criteria: age 60 or older; primary or recurrent sub-, juxta-, or extrafoveal CNV secondary to AMD; CNV including retinal angiomatous proliferation, that may benefit from treatment; BCVA of 78 to 20 letters; size of lesion $<12$ disc areas

Exclusion criteria: subretinal haemorrhage involving $\geq 70 \%$ of the lesion area; subfoveal fibrosis or atrophy in the study eye; CNV of other pathogenesis; history of ocular anti-VEGF treatment within 2 months, triamcinolone within 6 months, or laser treatment within 1 month; active intraocular inflammation, retinal pigment epithelial tear involving the macula, or vitreous haemorrhage obscuring view of the posterior pole in the study eye; IOP > $25 \mathrm{mmHg}$; cataract extraction within 3 months; myopia > 8 dioptre; hypersensitivity or allergy to testing agents; mentally or physically unable to participate; serious disease with probability of death during the study

Equivalence of baseline characteristics: not known

Diagnoses in participants: not known 
BRAMD

Interventions

Intervention 1: $1.25 \mathrm{mg}$ bevacizumab intravitreal injection monthly for 2 years

Intervention 2: $0.5 \mathrm{mg}$ ranibizumab intravitreal injection monthly for 2 years

Length of follow-up: 12 months

Outcomes

Primary outcomes, as defined: primary outcome was the change in BCVA in the study eye from baseline to 12 months. The non-inferiority margin was set at 4 letters

Secondary outcomes, as defined: proportion of participants losing fewer than 15 letters at 12 months (responders); proportion of participants with a loss or gain of BCVA less than 15 letters at 12 months (stabilisers); proportion of participants losing 15 letters or more of BCVA at 12 months (losers); proportion of participants gaining 15 letters or more of BCVA at 12 months (gainers); incidence of fluorescein leakage at 4 and 12 months; change in total area of CNV, total area of leakage from CNV, and total lesion area at 12 months, as determined by the reading centre; absolute and per cent change in retinal thickness, as measured by OCT at 4 and 12 months; proportion of drop-outs before the final 12-month assessment; proportion of non-responders at the 4-month assessment; costs of the 2 treatments

Adverse events: classification method definition not reported

Safety assessments: number of adverse events at 12 months

Intervals at which outcome assessed: 12 months

Notes

Full study name: Comparison of Bevacizumab (Avastin) and Ranibizumab (Lucentis) in Exudative Age-related Macular Degeneration (BRAMD)

Type of study: published as an abstract (ARVO 2014)

Funding sources: sponsors/collaborators: Academic Medical Centre (AMC), Department of Ophthalmology; The Netherlands Organization for Health Research and Development

Declarations of interest: not known

Study period: March 2009; primary completion date of July 2013

Reported subgroup analyses: not known

Risk of bias

\begin{tabular}{l|ll}
\hline Bias & Authors' judgement & Support for judgement \\
\hline $\begin{array}{l}\text { Random sequence generation (selection } \\
\text { bias) }\end{array}$ & Unclear risk & Not reported \\
\hline $\begin{array}{l}\text { Allocation concealment (selection bias) } \\
\begin{array}{l}\text { Blinding of participants and personnel } \\
\text { (performance bias) } \\
\text { All outcomes }\end{array}\end{array}$ & Unclear risk & Not reported \\
\hline
\end{tabular}

Blinding of outcome assessment (detection Unclear risk bias)

Reported as double-masked but methods

All outcomes

Incomplete outcome data (attrition bias) Unclear risk All outcomes

Reported as double-masked but methods not known

Systemic safety of bevacizumab versus ranibizumab for neovascular age-related macular degeneration (Review)

Copyright $\Subset 2014$ The Cochrane Collaboration. Published by John Wiley \& Sons, Ltd. 
BRAMD (Continued)

\begin{tabular}{lll} 
Selective reporting (reporting bias) & Unclear risk & $\begin{array}{l}\text { We searched and collected unpublished } \\
\text { data on death and All SSAE }\end{array}$ \\
\hline Other bias & Unclear risk & $\begin{array}{l}\text { Unpublished study with limited informa- } \\
\text { tion available }\end{array}$ \\
\hline Adverse event definition & Unclear risk & Not reported \\
\hline Method of adverse event assessment & Unclear risk & Not reported
\end{tabular}

\section{CATT}

Methods

Number randomised (total and per group): 1208 participants randomly assigned to study treatment; number of participants randomised per group not reported

Exclusions after randomisation: 1 study centre (23 participants) was excluded due to protocol violations

Number analysed (total and per group): 1105 total participants; 284 in ranibizumab monthly group, 265 in bevacizumab monthly group, 285 in ranibizumab as needed group, and 271 in bevacizumab as needed group

Unit of analysis: individuals (1 study eye per participant)

Losses to follow-up: 80 total participants: 17 in ranibizumab monthly group (4 died and 13 with missing data), 21 in bevacizumab monthly group ( 4 died and 17 with missing data), 13 in ranibizumab as needed group ( 5 died and 8 with missing data), and 29 in bevacizumab as needed group ( 11 died and 18 with missing data)

Compliance: limited information given: mean of 11.7 treatments given for ranibizumab monthly group and mean of 11.9 treatments given for bevacizumab monthly group

Intention-to-treat analysis: no, 103 participants enrolled and randomised were not included in the analyses

Reported power calculation: yes, sample of 277 participants per group for power of 90\%

Unusual study design: non-inferiority design, 4 arms, 6 pairwise comparisons planned; at 1 year, participants in the monthly dose treatment groups were re-randomised to either continue with monthly injections or switch to as needed injections of the same treatment drug

Participants

Country: USA

Age: mean was 79 years in ranibizumab monthly group, 80 years in bevacizumab monthly group, 78 years in ranibizumab as needed group, and 79 years in bevacizumab as needed group

Gender (per cent): 732/1185 (61.8\%) women and 453/1185 (38.2\%) men

Inclusion criteria: age 50 or older; 1 study eye per participant with untreated active CNV due to AMD (based on presence of leakage as seen by fluorescein angiography and of fluid as seen by OCT); VA of 20/25 to 20/320 on electronic visual acuity testing

Exclusion criteria: fibrosis or atrophy in centre of fovea in the study eye; CNV in either eye due to other causes; retinal pigment epithelial tear involving the macula; any concurrent intraocular condition in the study eye (e.g., cataract or diabetic retinopathy) that, in the opinion of the investigator, could either require medical or surgical intervention or contribute to VA loss during the 3-year follow-up period; active or recent (within 4 
weeks) intraocular inflammation; current vitreous haemorrhage in the study eye; history of rhegmatogenous retinal detachment or macular hole; active infectious conjunctivitis, keratitis, scleritis, or endophthalmitis; spherical equivalent $>8$ dioptres; intraocular surgery (including cataract surgery) in the study eye within 2 months; uncontrolled glaucoma; participants unable to be photographed to document $\mathrm{CNV}$, due to known allergy to fluorescein dye, lack of venous access or cataract obscuring the CNV; premenopausal women not using adequate contraception; pregnancy or lactation; history of other disease, metabolic dysfunction, physical examination finding, or clinical laboratory finding giving reasonable suspicion of a disease or condition that contraindicates the use an investigational drug or that might affect interpretation of the results of the study or render the subject at high risk for treatment complications; current treatment for active systemic infection; uncontrolled concomitant diseases such as cardiovascular disease, nervous system, pulmonary, renal, hepatic, endocrine, or gastrointestinal disorders; history of recurrent significant infections or bacterial infections; inability to comply with study or follow-up procedures

Equivalence of baseline characteristics: a slightly higher percentage of participants in bevacizumab monthly group had history of transient ischaemic attack $(8.7 \%$ compared with $4 \%$ in ranibizumab monthly group, $4 \%$ in ranibizumab as needed group, and 6. $3 \%$ in bevacizumab as needed group)

Diagnoses in participants: 688/1185 (58\%) had active neovascular AMD with CNV in foveal centre; $315 / 1185(27 \%)$ had fluid in foveal centre; $93 / 1185(8 \%)$ had haemorrhage in foveal centre; 71/1185 (6\%) had other foveal centre involvement; and 18/1185 (1. $5 \%$ ) had no CNV or not possible to grade

History of cardiovascular events at baseline: (bevacizumab, ranibizumab): MI 10.9\%, $13 \%$; stroke 6\%, 5.8\%; TIA 4\%, 7.5\%

Interventions

Intervention 1: $0.5 \mathrm{mg}$ intravitreal ranibizumab on a fixed schedule of every 4 weeks for 1 year, at 1 year, re-randomisation to ranibizumab every 4 weeks or as needed

Intervention 2: $1.25 \mathrm{mg}$ intravitreal bevacizumab on a fixed schedule of every 4 weeks for 1 year, at 1 year, re-randomisation to bevacizumab every 4 weeks or as needed

Intervention 3: $0.5 \mathrm{mg}$ intravitreal ranibizumab as needed for 2 years

Intervention 4: $1.25 \mathrm{mg}$ intravitreal bevacizumab as needed for 2 years

Length of follow-up:

Planned: 12 months for primary analysis; 24 months for secondary analyses, with modifications to 2 intervention arms as described above

Actual: 12 months for primary analysis; 24 months for secondary analyses

Outcomes

Primary outcome, as defined: change in visual acuity from baseline at 12 months with a non-inferiority margin of 5 letters

Secondary outcomes: proportion of eyes with 15-letter change, number of injections, OCT measured change in foveal thickness, change in lesion size on OCT and also on fluorescein angiography, incidence of ocular and systemic adverse events, and annual drug cost

Intervals at which outcome were assessed: weeks 4, 12, 24, 36, 52 during first year for visual acuity; weeks 4, 8, 12, 24, 52 for changes on OCT

Notes

Full study name: Comparison of Age-related macular degeneration Treatment Trials Type of study: published

Funding: National Eye Institute, National Institutes of Health, US 
Declarations of interest: 1 investigator reported receiving consulting fees from GlaxoSmithKline and another consulting fees from Neurotech and SurModics

Study period: accrual February 2008 through December 2009; follow-up through December 2011

Reported subgroup analyses: none

\section{Risk of bias}

\begin{tabular}{l|l|l}
\hline Bias & Authors' judgement & Support for judgement \\
\hline $\begin{array}{l}\text { Random sequence generation (selection } \\
\text { bias) }\end{array}$ & Low risk & "Patients were randomly assigned to 1 of \\
& & $\begin{array}{l}4 \text { study groups. Randomization schedules } \\
\text { were stratified according to clinical centre } \\
\text { with the use of a permuted-block method } \\
\text { with randomly chosen block sizes." }\end{array}$
\end{tabular}

Allocation concealment (selection bias) Low risk

Blinding of participants and personnel High risk (performance bias)

All outcomes
Web-based data entry system was used to allocate participants to treatment groups

Initially, participants were masked to which drug they received, but not to the treatment schedule. The study investigators noted that "insurance and billing documents specified ranibizumab but not studysupplied bevacizumab. Therefore, patients may have learned or deduced their assigned drug from these financial documents.”

Electronic Visual Acuity system (computerised testing) was used for primary outcome. Retinal centre personnel were masked. Adverse event reporting was unmasked, but medical monitor who evaluated serious adverse events was masked

7.3\% missing data excluding deaths, but we discussed this issue with the authors and concluded that active AE monitoring limited the risk of bias related to missing data on AEs

Incomplete outcome data (attrition bias) Low risk All outcomes

Low risk

Low risk
Reported outcomes: published data on death and All SSAE

Primary and secondary outcomes, specified a priori, for 1 year of follow-up were reported 
GEFAL

Methods
Number randomised (total and per group): 501 participants randomly assigned to study treatment; 255 in bevacizumab group and 246 in ranibizumab group

Exclusions after randomisation: 16 participants excluded because they received no injection ( 9 in bevacizumab group and 7 in ranibizumab group)

Number analysed (total and per group): 485 participants (246 in bevacizumab group and 239 in ranibizumab group) for safety analysis at 1 year; 404 participants (207 in bevacizumab group and 197 in ranibizumab group) for analysis on visual acuity at 1 year; most data analysed for 374 participants (191 in bevacizumab group and 183 in ranibizumab group) with available baseline BCVA data, at least 10 months follow-up, and did not have major deviations from the study protocol

Unit of analysis: individuals (1 study eye per participant)

Losses to follow-up: 81 total participants: 39 in bevacizumab group and 42 in ranibizumab group; additional 30 participants (16 in bevacizumab group and 14 in ranibizumab group) excluded from most analyses due to protocol violations

Compliance: $374 / 501$ participants completed the study without major protocol violations

Intention-to-treat analysis: no, not all participants enrolled and randomised were included in the analyses

Reported power calculation: yes, sample of 200 participants per group for power of $90 \%$ to detect 15 letters changes in BCVA

Unusual study design: non-inferiority design
Participants
Country: France (38 study centres)

Age: mean age for 374 participants without major protocol violations was 79 years

Gender (per cent): 248/374 (66\%) women and 126/374 (34\%) men

Inclusion criteria: age 50 or older; active subfoveal neovascular AMD (1 study eye eligible in bilateral cases); lesion size $<12$ disc areas; recent development of lesion in cases of occult neovessels; BCVA of 20/32 to 20/320 on ETDRS scale

Exclusion criteria: subretinal haemorrhage reaching foveal centre and $>50 \%$ of the lesion area; fibrosis or atrophy in centre of fovea in the study eye; CNV of other pathogenesis; retinal pigment epithelial tear reaching the macula; previous or current treatment with intravitreal anti-VEGF therapy; history of treatment 3 months prior or intraocular surgery 2 months prior to first study injection; history of photocoagulation or intravitreal medical device in the study eye; ocular or periocular infection; intraocular inflammation; diabetic retinopathy; history of autoimmune or idiopathic uveitis; IOP $\geq$ $25 \mathrm{mmHg}$ with topical hypotensive therapy; aphakia or lack of lens capsule in the study eye; known illness or condition requiring intraocular surgery within 12 months; known hypersensitivity to study drugs or allergy to agents used for ocular testing; uncontrolled arterial hypertension; history of treatment with systemic bevacizumab; premenopausal women not using adequate contraception; involvement in another clinical study; not part of French national health insurance programme 
GEFAL (Continued)

Equivalence of baseline characteristics: yes

Diagnoses in participants: $354 / 374$ (95\%) had intraretinal and/or subretinal fluid on OCT

History of cardiovascular events at baseline: (bevacizumab, ranibizumab): hypertension: $62.3 \%, 51.4 \%$; MI 5.2\%, 1.6\%; stroke 3.7\%, 3.7\%; TIA 0.5\%, 0\%

Interventions

Intervention 1: $1.25 \mathrm{mg}$ intravitreal bevacizumab every month for first 3 months; retreatment afterwards based on OCT or VA changes

Intervention 2: $0.50 \mathrm{mg}$ intravitreal ranibizumab every month for first 3 months; retreatment afterwards based on OCT or VA changes

Length of follow-up:

Planned: 1 year

Actual: 1 year

Outcomes

Primary outcome, as defined: mean change in BCVA at 1 year (at least 10 months after inclusion), as measured on an ETDRS chart

Secondary outcomes, as defined in published reports: visual acuity outcomes at 1 year: BCVA, change in BCVA, proportion with gain of $\geq 15$ letters, proportion with loss of $\geq 15$ letters, proportion with gain of $\geq 5$ letters, proportion with loss of $\geq$ 5 letters; change in CNV area between the baseline and final evaluations; presence of intraretinal and/or subretinal fluid; presence of pigment epithelial detachment; central subfield macular thickness; change in central subfield macular thickness; dye leakage on angiogram; number of injections; model of OCT equipment; adverse events

Secondary outcomes, as defined in trial registry: efficacy of treatments at 1 year; proportions of ocular and systemic adverse events at 1 year; average number of injections and time before re-injection during 1 year; drug profiles in blood and aqueous humor of a subset of 20 participants at 3 months; medico-economic impact of treatments at 1 year

Intervals at which outcome were assessed: monthly through 12 months

Notes

Full study name: Groupe d'Etude Français Avastin versus Lucentis dans la DMLA néovasculaire

Type of study: published

Funding sources: French Ministry of Health (Programme Hospitalier de Recherche Clinique National 2008); the French Health Insurance System co-financed the study and funded study drugs

Declarations of interest: 4 authors declared disclosures as principal investigators for trials sponsored by Novartis, Bausch \& Lomb, Théa, and Alcon; serving on advisory boards for Alcon, Allergan, Bayer, Bausch \& Lomb, Novartis, and Théa; receiving lecture fees from Alcon, Allergan, Bayer, Bausch \& Lomb, Heidelberg Engineering, the Krys group, Novartis, Théa, and Zeiss; receiving consulting fees from Novartis, Bayer, and Allergan; or receiving honoraria from Novartis, Bayer, and Allergan; the other 4 authors declared no conflicts of interests

Study period: random enrolment 24 June 2009 to 9 November 2011

Reported subgroup analyses: none 
GEFAL (Continued)

\begin{tabular}{l|ll}
$\begin{array}{l}\text { Random sequence generation (selection } \\
\text { bias) }\end{array}$ & Low risk & $\begin{array}{l}\text { "The randomization was stratified by cen- } \\
\text { tre and visual acuity (threshold: } 20 / 100) \\
\text { Local hospital pharmacies were responsible } \\
\text { for randomizing patients in each centre us- } \\
\text { ing pre-established lists." }\end{array}$ \\
\hline $\begin{array}{l}\text { Allocation concealment (selection bias) } \\
\text { Low risk }\end{array}$ & L & $\begin{array}{l}\text { Randomisation was controlled by the phar- } \\
\text { macy service and was not accessible to in- } \\
\text { vestigators (authors communication) }\end{array}$
\end{tabular}

Blinding of participants and personnel Low risk (performance bias)

All outcomes

"Identical syringes were masked and delivered by local hospital pharmacies after aseptic preparation in authorized, centralized drug-preparation units, using vials of Avastin $100 \mathrm{mg} / \mathrm{ml}$ and Lucentis $10 \mathrm{mg} /$ ml."

"The main strength of the GEFAL trial is that the study remained effectively doublemasked, unlike CATT in which some participants received billing information and IVAN in which the masking differed between centres (some treating teams were aware of treatment allocation)."

Blinding of outcome assessment (detection Low risk

Masking of outcome assessors achieved (aubias) thors' communication)

All outcomes

Incomplete outcome data (attrition bias) Low risk All outcomes

4.3\% missing data excluding deaths

Selective reporting (reporting bias) Low risk

Reported outcomes: published data on death and All SSAE

Other bias

Low risk

Differences in outcomes between the trial registration and published 1-year results papers included:

1) secondary visual acuity and morphology outcomes were specified clearly in the paper, but only described as 'efficacy of treatments' in the trial registration

2) the published paper included model of OCT equipment as outcome, whereas the trial registration did not

3) the trial registration included time before re-injection during 1 year, drug profiles in blood and aqueous humor of a subset of 20 participants at 3 months, and medico-economic impact of treatments as outcomes, 
GEFAL (Continued)

whereas the published paper did not

\begin{tabular}{lll}
\hline Adverse event definition & Low risk & $\begin{array}{l}\text { Pre-specified, MedDRA reporting system } \\
\text { adopted }\end{array}$ \\
\hline Method of adverse event assessment & Low risk & Active monitoring of AEs \\
\hline
\end{tabular}



group completed first 3 injections and were randomised to continue or discontinue treatment: 157 continued ranibizumab; 155 discontinued ranibizumab; 149 continued bevacizumab; and 145 discontinued bevacizumab

Exclusions after randomisation: 18 participants did not receive treatment and were excluded after randomisation to drug treatment ( 9 in ranibizumab group and 9 in bevacizumab group)

Number analysed (total and per group):

At 1-year follow-up: 561 total participants at 1 year; 141 in continued ranibizumab group; 146 in discontinued ranibizumab group; 136 in continued bevacizumab group; and 138 in discontinued bevacizumab group

At 2-year follow-up: 525 total participants at 1 year; 134 in continued ranibizumab group; 137 in discontinued ranibizumab group; 127 in continued bevacizumab group; and 127 in discontinued bevacizumab group

Unit of analysis: individuals (1 study eye per participant)

Losses to follow-up:

At 1-year follow-up: 49 total participants: 4 participants receiving treatment withdrew prior to completing third injection ( 2 in ranibizumab group and 2 in bevacizumab group); 45 participants randomised to regimen groups exited trial before 1 year (16 in continued ranibizumab group; 9 in discontinued ranibizumab group; 13 in continued bevacizumab group; and 7 in discontinued bevacizumab group)

At 2-year follow-up: 85 total participants: 5 participants receiving treatment withdrew prior to completing third injection ( 2 in ranibizumab group and 3 in bevacizumab group); 80 participants randomised to regimen groups exited trial before 2 years ( 23 in continued ranibizumab group; 18 in discontinued ranibizumab group; 21 in continued bevacizumab group; and 18 in discontinued bevacizumab group)

Compliance: the wrong study drug was administered twice during the first year:

At 1-year follow-up: adherence was 6576/6699 (98\%) scheduled injections received At 2-year follow-up: adherence was 12761/14640 (87\%) scheduled injections received Intention-to-treat analysis: no, 67 participants enrolled and randomised were not included in the analyses at 1 year and 103 at 2 years

Reported power calculation: yes, sample of 600 participants per group for power of $90 \%$ to detect non-inferiority

Unusual study design: non-inferiority design; 2 × 2 factorial design - randomisation in 2 stages: first randomised to drug treatment (ranibizumab or bevacizumab), then to treatment regimen (continue monthly injections or discontinue monthly injections and switch to as needed injections given in 3-month cycles); results reported only as ranibizumab versus bevacizumab and continuous versus discontinuous with any component of the neovascular lesion (CNV, blood, serous pigment epithelial detachment, elevated blocked fluorescence) involving the centre of the fovea, confirmed by fluorescein angiography; BCVA of 25 letters or greater on the ETDRS chart (measured at 1 metre) 
Exclusion criteria: neovascular lesion of $50 \%$ or more fibrosis or blood; more than 12 disc diameters; argon laser treatment in study eye within 6 months; presence of thick blood involving the centre of the fovea; presence of other active ocular disease causing concurrent vision loss; myopia 8 or more dioptres; previous treatment with PDT or a VEGF inhibitor in study eye; women pregnant, lactating, or of child bearing potential; men with a spouse or partner of child bearing potential

Equivalence of baseline characteristics: yes

Diagnoses in participants: 301/610 (58\%) had neovascular AMD with CNV in foveal centre; $308 / 610$ (54\%) had fluid in foveal centre; $90 / 610$ (16\%) had haemorrhage in foveal centre; 75/610 (13\%) had other foveal centre involvement; and 15/610 (3\%) had no $\mathrm{CNV}$ or not possible to grade

History of cardiovascular events at baseline (bevacizumab, ranibizumab): hypertension not reported, MI 7.4\%, 7.6\%, stroke 2.2\%, 2.3\%, TIA 6.4\%, 3\%

Interventions

Intervention 1: $0.5 \mathrm{mg}$ intravitreal ranibizumab monthly for 2 years

Intervention 2: $1.25 \mathrm{mg}$ intravitreal bevacizumab monthly for 2 years

Intervention 3: after first 3 monthly $0.5 \mathrm{mg}$ intravitreal ranibizumab injections, monthly treatment was discontinued and treatment was given as needed in cycles of 3 monthly doses

Intervention 4: after first 3 monthly $1.25 \mathrm{mg}$ intravitreal bevacizumab injections, monthly treatment was discontinued and treatment was given as needed in cycles of 3 monthly doses

Length of follow-up:

Planned: 2 years

Actual: 2 years

Primary outcome, as defined: best corrected distance visual acuity measured as ETDRS letters at 2 years

Secondary outcomes, as defined in protocol: at 1-year and 2-year follow-up - frequencies of adverse effects of treatment; generic and vision-specific health-related quality of life; treatment satisfaction; cumulative resource use/cost and cost-effectiveness; clinical measures of vision (contrast sensitivity measured with Pelli-Robson charts, near visual acuity measured by Bailey-Love near reading cards, and reading speed measured with Belfast reading charts); lesion morphology (fluorescein angiography and OCT); distance visual acuity at 1 year; survival free from treatment failure

Exploratory analysis: association between serum markers and cardiovascular serious adverse events

Intervals at which outcome were assessed: monthly through 24 months; various data were collected at every visit depending on assessment schedule and regimen group

Notes

Full study name: alternative treatments to Inhibit VEGF in Age-related choroidal Neovascularisation

Type of study: published

Funding sources: National Institute for Health Research Health Technology Assessment programme, UK

Declarations of interest: various authors reported being principal investigators of trials sponsored by Novartis; attending and being remunerated for attendance at advisory boards for Novartis, Bayer, Neovista, Oraya, Allergan, and/or Bausch and Lomb; being employed by institution that has received payments from Novartis, Bayer, Neovista, 
Oraya, Alcon, and/or Pfizer; receiving honoraria from Novartis for lecture and/or teaching fees from Janssen-Cilag

Study period: random enrolment 27 March 2008 to 15 October 2010

Reported subgroup analyses: none

Risk of bias

\begin{tabular}{|c|c|c|}
\hline Bias & Authors' judgement & Support for judgement \\
\hline $\begin{array}{l}\text { Random sequence generation (selection } \\
\text { bias) }\end{array}$ & Low risk & $\begin{array}{l}\text { "randomised allocations were computer } \\
\text { generated by a third party in blocks and } \\
\text { stratified by centre." } \\
\text { "Randomisation was stratified by centre } \\
\text { and was blocked to ensure roughly equal } \\
\text { numbers of participants per group within } \\
\text { a centre." }\end{array}$ \\
\hline
\end{tabular}

Allocation concealment (selection bias) Low risk

"Research teams at sites recruited participants, and accessed a password-protected website to randomize participants. Allocations were concealed until participants' eligibility and identities were confirmed."

"Allocations were computer generated and concealed with an internet-based system (Sealed Envelope, London, UK). Staff in participating centres accessed the website and, on entering information to confirm a participant's identity and eligibility, were provided with the unique study number."

Blinding of participants and personnel Low risk (performance bias)

All outcomes
From study protocol:

"Participants, clinicians and trial personnel will be masked to the VEGF inhibitor to which a participant is assigned."

"We have chosen not to mask participants, clinicians and trial personnel to whether patients are allocated to continue or stop treatment at 3 months."

"We intended that drug allocation should be concealed by having separate masked assessment and unmasked treating teams. This system was achieved by 14 sites. At the other 9 sites, staffing levels could not support this system and an unmasked staff member prepared ranibizumab in a syringe identical to those containing bevacizumab and did not perform assessments."

"Lesion morphology was assessed by inde- 


\begin{tabular}{|c|c|c|}
\hline & & $\begin{array}{l}\text { pendent graders masked to drug and treat- } \\
\text { ment regimen." } \\
\text { From study protocol: } \\
\text { "We have chosen not to mask participants, } \\
\text { clinicians and trial personnel to whether } \\
\text { patients are allocated to continue or stop } \\
\text { treatment at } 3 \text { months." }\end{array}$ \\
\hline $\begin{array}{l}\text { Incomplete outcome data (attrition bias) } \\
\text { All outcomes }\end{array}$ & Low risk & $\begin{array}{l}13.8 \% \text { missing data at } 2 \text { years excluding } \\
\text { deaths. After discussion with trial authors, } \\
\text { we concluded that active AE monitoring } \\
\text { limited the risk of bias related to missing } \\
\text { data on AEs }\end{array}$ \\
\hline Selective reporting (reporting bias) & Unclear risk & $\begin{array}{l}\text { Reported outcomes: published data on } \\
\text { death and All SSAE }\end{array}$ \\
\hline Other bias & Low risk & $\begin{array}{l}\text { Differences between the protocol and pub- } \\
\text { lished } 1 \text {-year and } 2 \text {-year results papers in- } \\
\text { cluded: } \\
\text { 1) } 2 \text { secondary outcomes in the protocol } \\
\text { were not listed in the paper: treatment sat- } \\
\text { isfaction and survival free from treatment } \\
\text { failure; and } \\
\text { 2) exploratory (serum) analysis in protocol } \\
\text { upgraded to a secondary outcome in paper }\end{array}$ \\
\hline Adverse event definition & Low risk & $\begin{array}{l}\text { Pre-specified, MedDRA reporting system } \\
\text { adopted }\end{array}$ \\
\hline Method of adverse event assessment & Low risk & Active monitoring of AEs \\
\hline
\end{tabular}

\section{LUCAS}

Methods

Number randomised (total and per group): 420 participants

Exclusions after randomisation: not known

Number analysed (total and per group): 218 to $0.5 \mathrm{mg}$ ranibizumab group, and 2014 to bevacizumab $1.25 \mathrm{mg}$

Unit of analysis: not known

Losses to follow-up: not known

Compliance: "more than $90 \%$ of patients in each treatment group remained in the study at 12 months, and approximately 80 to $90 \%$ remained at 24 months"

Intention-to-treat analysis: not known

Reported power calculation: not known

Unusual study design: none known

Participants

Country: Norway

Age: not known

Gender (per cent): not known

Systemic safety of bevacizumab versus ranibizumab for neovascular age-related macular degeneration (Review) 
Inclusion criteria: age 50 or older; unilateral or bilateral neovascular AMD (1 study eye eligible in bilateral cases); untreated CNV including retinal angiomatous proliferation, with oedema involving the fovea as assessed by fluorescein angiography and OCT; BCVA of $20 / 25$ to $20 / 320$

Exclusion criteria: subretinal haemorrhage and/or fibrosis involving $\geq 50 \%$ of the lesion area; CNV of other pathogenesis; previous treatment for CNV; history of antiVEGF treatment in non-study eye within 4 weeks; intraocular surgery or laser treatment within 3 months; infection in either eye; active uveitis or intraocular inflammation; retinal disease that may lead to vision loss in the study eye; impaired visualisation of the retina precluding adequate diagnosis; IOP $\geq 25 \mathrm{mmHg}$ or uncontrolled glaucoma; cataract requiring surgery within 2 years; history of treatment with systemic anti-VEGF drugs; premenopausal women not using adequate contraception or nursing; mentally or physically unable to participate; serious disease with probability of death during the study; involvement in another clinical study or use of investigational drugs involving the macula in the study eye

Equivalence of baseline characteristics: not known

Diagnoses in participants: not known

Interventions

Intervention 1: $25 \mathrm{mg} / \mathrm{ml}$ intravitreal bevacizumab administered following the "inject and extend" principle

Intervention 2: $10 \mathrm{mg} / \mathrm{ml}$ intravitreal ranibizumab administered following the "inject and extend" principle

Length of follow-up: 2 years

Outcomes

Primary outcomes, as defined: mean change in visual acuity at 1 and 2 years, as measured on an ETDRS chart (non-inferiority limit of 5 letters)

Secondary outcomes, as defined: number of treatments at 1 and 2 years; proportions of participants losing fewer than 15 letters at 1 and 2 years, as measured on an ETDRS chart; macular morphology at 2 years, as measured by fluorescein angiography and OCT; adverse events at 2 years; number of non-responders at 2 years

Adverse events:classification used not known

Safety assessments: not known

Intervals at which outcome assessed: not known

Notes

Full study name: Lucentis Compared to Avastin Study (LUCAS)

Type of study: unpublished (oral communication, American Academy of Ophthalmology)

Funding sources: Ullevaal University Hospital, Norway

Declarations of interest: not available

Study period: March 2009; primary completion date of July 2013

Reported subgroup analyses: not known

\section{Risk of bias}

\begin{tabular}{l|l|l} 
Bias & Authors' judgement & Support for judgement \\
\hline $\begin{array}{l}\text { Random sequence generation } \\
\text { bias) }\end{array}$ & Nelection & Unclear risk
\end{tabular}




\section{LUCAS (Continued)}

\begin{tabular}{l|ll}
\hline Allocation concealment (selection bias) & Unclear risk & Not reported \\
\hline $\begin{array}{l}\text { Blinding of participants and personnel } \\
\text { (performance bias) } \\
\text { All outcomes }\end{array}$ & Unclear risk & $\begin{array}{l}\text { Reported as double-masked but methods } \\
\text { not known }\end{array}$ \\
\hline $\begin{array}{l}\text { Blinding of outcome assessment (detection } \\
\text { bias) } \\
\text { All outcomes }\end{array}$ & Unclear risk & $\begin{array}{l}\text { Reported as double-masked but methods } \\
\text { not known }\end{array}$ \\
\hline $\begin{array}{l}\text { Incomplete outcome data (attrition bias) } \\
\text { All outcomes }\end{array}$ & Unclear risk & $\begin{array}{l}\text { Reported as double-masked but methods } \\
\text { not known }\end{array}$ \\
\hline $\begin{array}{l}\text { Selective reporting (reporting bias) } \\
\text { Uther bias }\end{array}$ & Unclear risk & $\begin{array}{l}\text { Unpublished data on death. We searched } \\
\text { and derived data for All SSAE }\end{array}$ \\
\hline $\begin{array}{l}\text { Adverse event definition } \\
\text { Method of adverse event assessment }\end{array}$ & Unclear risk & $\begin{array}{l}\text { Unpublished study with limited informa- } \\
\text { tion available }\end{array}$ \\
\hline
\end{tabular}

\section{MANTA}

Methods

Number randomised (total and per group): 321 participants randomly assigned to study treatment; number per group not reported

Exclusions after randomisation: 4 participants ( 3 due to receiving the wrong drug and 1 because the participant received prior treatment and was not eligible)

Number analysed (total and per group): 317 total participants; 154 in bevacizumab group and 163 in ranibizumab group

Unit of analysis: individuals (1 study eye per participant)

Losses to follow-up: 69 participants: reasons for losses to follow-up not reported (33 in bevacizumab group, 36 in ranibizumab group)

Compliance: $248 / 317$ participants completed the study

Intention-to-treat analysis: no, 4 participants enrolled and randomised were not included in analysis; data imputed using last observation carried forward method for 69 participants lost to follow-up

Reported power calculation: yes, sample of 320 participants, "95\% power to detect a significant difference between ranibizumab and bevacizumab assuming a seven letters increase in visual acuity with ranibizumab and no change in visual acuity with bevacizumab". The assumption of no change in visual acuity with bevacizumab is unrealistic Unusual study design: non-inferiority design

Participants

Country: 10 clinical centres in Austria

Age: mean 76.7 years in bevacizumab group and 77.6 years in ranibizumab group Gender (per cent): $115 / 317$ (36.3\%) men and 202/317 (63.7\%) women

Inclusion criteria: age 50 or older; active primary or recurrent subfoveal lesion with 
CNV, measured by fluorescein angiography or OCT; BCVA in study eye between 20/ 40 to 20/320, measured by ETDRS charts

Exclusion criteria: previous treatment for $\mathrm{CNV}$ or $\mathrm{AMD}$; prior treatment with any intravitreal drug or verteporfin PDT in study eye; prior treatment with systemic bevacizumab; prior treatment with any intravitreal drug or verteporfin PDT in non-study eye within 3 months; laser photocoagulation in study eye within 1 month; participation in another clinical trial within 1 month; subfoveal fibrosis or atrophy $>50 \%$ in study eye; CNV in either eye due other causes than AMD; RPE tear involving macula of study eye; history of uncontrolled glaucoma or concurrent intraocular condition in study eye; pregnancy; allergy to fluorescein; inability to comply with study procedures

Equivalence of baseline characteristics: yes

Diagnoses in participants: active primary or recurrent subfoveal CNV

History of cardiovascular events at baseline (bevacizumab, ranibizumab): participants with severe hypertension, MI, stroke, TIA were excluded

Interventions

Intervention 1: $1.25 \mathrm{mg}$ intravitreal bevacizumab every month for first 3 months; retreatment afterwards based on OCT or VA changes

Intervention 2: $0.5 \mathrm{mg}$ intravitreal ranibizumab every month for first 3 months; retreatment afterwards based on OCT or VA changes

Length of follow-up:

Planned: 12 months

Actual: 12 months

Primary outcomes, as defined: "mean change in BCVA between baseline and 1 year" Secondary outcomes, as reported: Kaplan-Meier proportions of the gain of 15 letters of vision, gain of 5 letters of vision, loss of 5 letters of vision, loss of 15 letters of vision; lesion size, assessed by fluorescein angiography; number of re-treatments; and retinal thickness, assessed by OCT

Adverse events: classification method definition not reported

Intervals at which outcome assessed: monthly through 12 months

Full study name: A Randomized Observer and Subject Masked Trial Comparing the Visual Outcome After Treatment With Ranibizumab or Bevacizumab in Patients With Neovascular Age-related Macular Degeneration Multicentre Anti VEGF Trial in Austria Type of study: published

Funding sources: Austrian ophthalmologic society; the Ludwig Boltzmann Institute of Retinology and Biomicroscopic Lasersurgery; the participating study centre sites

Declarations of interest: authors reported no competing interests

Study period: not reported

Reported subgroup analyses: none

\section{Risk of bias}

\begin{tabular}{l|l} 
Bias & Authors' judgement
\end{tabular}

Random sequence generation (selection Low risk bias)
"Randomisation was stratified according to the clinical centre using a permuted block method with a fixed block size of 20." 
MANTA

(Continued)

\begin{tabular}{|c|c|c|}
\hline Allocation concealment (selection bias) & Low risk & $\begin{array}{l}\text { "Eligible patients were randomised in a } 1: 1 \\
\text { ratio to one of two groups by members of } \\
\text { the Department of Clinical Pharmacology, } \\
\text { Medical University of Vienna, which was } \\
\text { otherwise not involved in the study." }\end{array}$ \\
\hline $\begin{array}{l}\text { Blinding of participants and personnel } \\
\text { (performance bias) } \\
\text { All outcomes }\end{array}$ & Low risk & $\begin{array}{l}\text { "All other personnel and the patients were } \\
\text { masked to treatment assignment." }\end{array}$ \\
\hline $\begin{array}{l}\text { Blinding of outcome assessment (detection } \\
\text { bias) } \\
\text { All outcomes }\end{array}$ & Low risk & $\begin{array}{l}\text { "The evaluating physician was masked to } \\
\text { treatment assignment, whereas the inject- } \\
\text { ing physician was not involved in the col- } \\
\text { lection of data." }\end{array}$ \\
\hline $\begin{array}{l}\text { Incomplete outcome data (attrition bias) } \\
\text { All outcomes }\end{array}$ & Unclear risk & $\begin{array}{l}21.5 \% \text { missing visual acuity data at } 1 \text { year. } \\
\text { Active monitoring of adverse events might } \\
\text { have limited the risk of bias related to miss- } \\
\text { ing data on adverse events }\end{array}$ \\
\hline Selective reporting (reporting bias) & Low risk & $\begin{array}{l}\text { Reported outcomes: published data on } \\
\text { death and All SSAE }\end{array}$ \\
\hline Other bias & Low risk & None observed \\
\hline Adverse event definition & Unclear risk & Not reported \\
\hline Method of adverse event assessment & Unclear risk & Not reported \\
\hline
\end{tabular}



cizumab group, 1 in ranibizumab group); 1 participant relocated (in bevacizumab group) ; and 2 participants died (both in bevacizumab group)

Compliance: $22 / 28$ participants completed the study

Intention-to-treat analysis: no, 6 participants enrolled and randomised were not included in analysis

Reported power calculation: yes, $79 \%$ power for sample size of 135 participants using 2 : 1 randomisation ratio. However, the number of enrolled participants seem not adequate to detect differences in adverse events

Unusual study design: although the target sample size was 135 , only 28 participants were evaluated

Age: not reported for 28 enrolled participants (mean 78 years for analysed bevacizumab group; mean 80 years for analysed ranibizumab group)

Gender (per cent): not reported for 28 enrolled participants (all men for analysed bevacizumab group; 6 men and 1 woman for analysed ranibizumab group)

Inclusion criteria: age 50 or older; presence of symptomatic CNV, confirmed by intravenous fluorescein angiogram and optical coherence tomography as affecting the foveal centre; ability to provide informed consent; willing to commit to regular clinic appointments and follow-up; original protocol specified baseline VA between 20/40 and 20/ 200 , later amended to include all baseline VAs equal to or better than 20/400

Exclusion criteria: previous treatment for wet AMD within the past year; presence of subretinal haemorrhage greater than $50 \%$ of the size of the lesion on fluorescein angiography, presence of advanced glaucoma; any coexisting macular disease causing decreased vision; history of malignant or uncontrolled hypertension; intraocular inflammation; history of thromboembolic phenomena; inability to provide informed consent; participation in another concurrent ophthalmic clinical trial

Equivalence of baseline characteristics: yes

Diagnoses in participants: AMD

Interventions

Intervention 1: $0.05 \mathrm{ml}$ intravitreal bevacizumab every month for first 3 months; retreatment afterwards based on OCT or VA changes

Intervention 2: $0.05 \mathrm{ml}$ intravitreal ranibizumab every month for first 3 months; retreatment afterwards based on OCT or VA changes

Length of follow-up:

Planned: 12 months

Actual: 12 months

Outcomes

Primary outcomes, as defined: visual acuity

Secondary outcomes, as reported: central foveal thickness by OCT, total number of injections; blood pressure measurements

Adverse events

Intervals at which outcome assessed: 1 week after injections to assess adverse events; 
Subramanian 2010 (Continued)

and monthly through 12 months

\begin{tabular}{ll}
\hline Notes & Type of study: published \\
& Funding sources: Veterans Affairs Boston Healthcare System, USA \\
Declarations of interest: "The authors declare no conflict of interest" \\
Study period: April 2007 to February 2009 \\
Reported subgroup analyses: none \\
\hline
\end{tabular}

Risk of bias

\begin{tabular}{|c|c|c|}
\hline Bias & Authors' judgement & Support for judgement \\
\hline $\begin{array}{l}\text { Random sequence generation (selection } \\
\text { bias) }\end{array}$ & Unclear risk & $\begin{array}{l}\text { "Patients were enrolled by a } 2: 1 \text { random- } \\
\text { ization to either the bevacizumab ( } 2 \text { ) or the } \\
\text { ranibizumab (1) arm of the study." Study } \\
\text { investigators were contacted, but could not } \\
\text { provide additional information as to how } \\
\text { the sequence was generated (email commu- } \\
\text { nication with Dr. Subramanian, dated } 16 \\
\text { May 2012) }\end{array}$ \\
\hline Allocation concealment (selection bias) & Low risk & $\begin{array}{l}\text { "The Research Pharmacist at the [Veterans } \\
\text { Affairs] Hospital Pharmacy was responsible } \\
\text { for randomization" and "all subjects were } \\
\text { assigned a study number." }\end{array}$ \\
\hline
\end{tabular}

Blinding of participants and personnel Low risk (performance bias)

Reported as "double-blind"; identical syAll outcomes ringes were used to administer agents, and study personnel in contact with participants were all masked

Blinding of outcome assessment (detection Low risk bias)

"As the only investigator with knowledge of subject assignments, the Research Pharmacist was, in turn, masked to all visual and anatomic outcomes to treatment. All other investigators, as well as other physicians, residents, and office personnel who may have inadvertently come in contact with study subjects, were masked to treatment assignments."

Incomplete outcome data (attrition bias) High risk

6 of $28(21 \%)$ participants enrolled were All outcomes not included in the analysis: 3 voluntarily dropped out; 1 relocated; and 2 died

Selective reporting (reporting bias) Unclear risk

Reported outcomes: published data on death and All SSAE

Systemic safety of bevacizumab versus ranibizumab for neovascular age-related macular degeneration (Review) 
Subramanian 2010 (Continued)

Other bias

Unclear risk
Quality of life reported as an outcome in the clinical trials register but not reported in the full-text publication

Trial stopped early for poor recruitment. Stopping characteristics:

- Interim analyses: after the first 28 participants enrolled (sample size planned 135 participants)

- Details of stop: "From April 2007 to February 2009, 28 patients were enrolled in the study. This relatively low number of patients enrolled over a 2-year period was likely due to two reasons. A larger than expected number of potential study participants was previously treated with visudyne or other anti-angiogenesis agents within the past 12 months, thus disqualifying them from the study. There was also a lower volume of AMD patients who presented to the Veterans Affairs and met inclusion criteria than initially anticipated. Both of these factors contributed to low enrolment. All those who met inclusion criteria were offered enrolment in the study, and a relatively high number (estimated $80 \%$ ) consented to participation."

\begin{tabular}{|c|c|c|}
\hline Adverse event definition & Unclear risk & Not reported \\
\hline
\end{tabular}

\begin{tabular}{l|l} 
Method of adverse event assessment $\quad$ Unclear risk & Not reported
\end{tabular}

\section{VIBERA}

Methods

Number randomised (total and per group): 161 participants (107 bevacizumab and 54 ranibizumab)

Exclusions after randomisation: 5

Number analysed (total and per group): 142,47 to $0.5 \mathrm{mg}$ ranibizumab group, and 95 to bevacizumab $1.25 \mathrm{mg}$

Unit of analysis: individuals (1 study eye per participant)

Losses to follow-up: none

Compliance: high

Intention-to-treat analysis: no, 142 participants included in the per protocol analysis Reported power calculation: 90\% (7 ETDRS letters)

Unusual study design: no

Participants

Country: Germany

Age: mean age 77 years

Gender (per cent): female 62\% 
Inclusion criteria: age 50 or older; visual impairment due to active primary or recurrent $\mathrm{CNV}$ associated with $\mathrm{AMD}$; classical or predominantly classic lesion with largest diameter of the subretinal neovascular membrane smaller than greatest distance between major temporal vascular arcades, minimally classic lesion, or occult lesion with no classic CNV; BCVA of 20/40 to 20/320

Exclusion criteria: subretinal haemorrhage involving $\geq 50 \%$ of the lesion area or $\geq$ 1 optic disc areas; subfoveal fibrosis or atrophy; CNV of other pathogenesis; previous treatment for $\mathrm{CNV}$ or treatment with any anti-angiogenic drugs; previous intravitreal drug delivery, laser photocoagulation, vitreoretinal surgery, submacular surgery, or other surgical intervention for AMD in the study eye; retinal pigment epithelial tear; active inflammation, vitreous haemorrhage, infectious conjunctivitis, keratitis, scleritis, or endophthalmitis; history of rhegmatogenous retinal detachment, macular hole, idiopathic or autoimmune-associated uveitis, or corneal transplant; aphakia or lack of posterior capsule in the study eye; $>-8$ dioptres of myopia; any intraocular condition that requires surgery or could lead to vision loss within 2 years; intraocular surgery in study eye within 2 months; uncontrolled glaucoma or history of glaucoma filtering surgery; impaired visualisation of the retina precluding adequate diagnosis; premenopausal women not using adequate contraception or nursing; active systemic infection or other disease, dysfunction, or finding to contraindicate participation; hypersensitivity to study drugs or allergy to agents used for ocular testing; involvement in another clinical study within 4 weeks; unwillingness or inability to comply with study

Equivalence of baseline characteristics: yes; no significant differences observed Diagnoses in participants: MI none, stroke: 1 (ranibizumab), TIA none

Outcomes

Notes
Primary outcomes, as defined: mean change in BCVA at 1 year, as measured on an ETDRS chart

Secondary outcomes, as defined: proportion of participants losing fewer than 15 letters at 2 years; proportion of participants with at least 3 months treatment-free in 2 years; number of doses of study drugs at 2 years; rate of drop-out at 2 years; number of nonresponders at 2 years; retinal lesions at 2 years; adverse events at 2 years; quality of life at 2 years

Adverse events: pre-specified, MedDRA reporting system

Safety assessments: adverse events at 1 year

Intervals at which outcome assessed: monthly

Full study name: Prevention of Vision Loss in Patients With Age-Related Macular Degeneration (AMD) by Intravitreal Injection of Bevacizumab and Ranibizumab (VIBERA)

Type of study: unpublished (first authors' personal communication)

Funding sources: Deutsche Stiftung für Chronisch Kranke (http://www.dsck.de)

Declarations of interest:

1 author declared no conflict of interest. 1 author declared disclosures for grants by the Deutsche Stiftung für Chronisch Kranke, Institutions of the German Health System (statutory health insurance, German Physicians Association)

Study period: August 2009; primary completion date of August 2012; study interrupted 
VIBERA

early due to loss of study medication production site

Reported subgroup analyses: not performed

\section{Risk of bias}

\begin{tabular}{ll|l} 
Bias & Authors' judgement & Support for judgement \\
\hline $\begin{array}{l}\text { Random sequence generation (selection } \\
\text { bias) }\end{array}$ & Low risk & $\begin{array}{l}2: 1 \text { randomisation, blockwise (by study } \\
\text { site) randomisation computer-generated in } \\
\text { the Department of Statistics }\end{array}$ \\
\hline Allocation concealment (selection bias) & Low risk & $\begin{array}{l}\text { Allocation list not accessible for study per- } \\
\text { sonnel nor participants, information on the } \\
\text { allocation code exclusively kept in the De- } \\
\text { partment of Statistics }\end{array}$ \\
\hline
\end{tabular}

Blinding of participants and personnel Low risk (performance bias)

Allocation information not accessible for All outcomes participants nor study personnel; identicallooking study medication

Blinding of outcome assessment (detection Low risk bias)

All outcomes

Allocation information not accessible for participants nor study personnel; identicallooking study medication

Incomplete outcome data (attrition bias) Low risk All outcomes

4 particpants excluded because of missing data at start or 1 year. No loss to follow-up. All drop-outs medically clarified

Selective reporting (reporting bias) Low risk

We searched and collected unpublished data on death and All SSAE

\begin{tabular}{ll} 
Other bias $\quad$ Low risk \\
\hline
\end{tabular}

All primary and secondary outcomes were reported

\begin{tabular}{|c|c|c|}
\hline Adverse event definition & Low risk & Pre-specified, MedDRA reporting system \\
\hline Method of adverse event assessment & Low risk & $\begin{array}{l}\text { Active monitoring of AEs: ophthalmologist } \\
\text { saw participants } 2 \text { to } 3 \text { days after injection, } \\
\text { participants were asked for AEs monthly } \\
\text { and when omitting study visits }\end{array}$ \\
\hline
\end{tabular}

AE: adverse event

AMD: age-related macular degeneration

ARVO: Association for Research in Vision and Ophthalmology

BCVA: best corrected visual acuity

CMT: central macular thickness

$\mathrm{CNV}$ : choroidal neovascularisation 
ETDRS: Early Treatment Diabetic Retinopathy Study IOP: intraocular pressure

MedDRA: Medical Dictionary for Regulatory Activities MI: myocardial infarction

SSAE: serious systemic adverse event

TIA: transient ischaemic attack

VA: visual acuity

VEGF: vascular endothelial growth factor 
DATA ANDANALYSES

Comparison 1. Bevacizumab versus ranibizumab, longest follow-up

\begin{tabular}{|c|c|c|c|c|}
\hline Outcome or subgroup title & $\begin{array}{l}\text { No. of } \\
\text { studies }\end{array}$ & $\begin{array}{c}\text { No. of } \\
\text { participants }\end{array}$ & Statistical method & Effect size \\
\hline 1 All-cause death & 8 & 3338 & Risk Ratio (M-H, Random, 95\% CI) & $1.10[0.78,1.57]$ \\
\hline $\begin{array}{l}2 \text { All serious systemic adverse } \\
\text { events }\end{array}$ & 9 & 3665 & Risk Ratio (M-H, Random, 95\% CI) & $1.08[0.90,1.31]$ \\
\hline 3 Arterial thromboembolic event & 6 & 3190 & Risk Ratio (M-H, Random, 95\% CI) & $0.92[0.62,1.37]$ \\
\hline 4 Gastrointestinal perforation & 1 & 161 & Risk Ratio (M-H, Random, 95\% CI) & $0.0[0.0,0.0]$ \\
\hline 5 Infection & 6 & 3190 & Risk Ratio (M-H, Random, 95\% CI) & $1.34[0.97,1.86]$ \\
\hline 6 Myocardial infarction & 6 & 3190 & Risk Ratio (M-H, Random, 95\% CI) & $0.84[0.42,1.66]$ \\
\hline 7 Neutropenia & 1 & 161 & Risk Ratio (M-H, Random, 95\% CI) & $0.0[0.0,0.0]$ \\
\hline 8 Non-ocular haemorrhage & 3 & 1256 & Risk Ratio (M-H, Random, 95\% CI) & $0.39[0.08,1.85]$ \\
\hline 9 Stroke & 6 & 3190 & Risk Ratio (M-H, Random, 95\% CI) & $0.83[0.42,1.66]$ \\
\hline $\begin{array}{l}10 \text { Treatment-related drug } \\
\text { discontinuation }\end{array}$ & 0 & 0 & Risk Ratio (M-H, Random, 95\% CI) & $0.0[0.0,0.0]$ \\
\hline $\begin{array}{l}11 \text { Vascular events associated with } \\
\text { anti-VEGF treatment }\end{array}$ & 3 & 1823 & Risk Ratio (M-H, Random, 95\% CI) & $1.03[0.52,2.05]$ \\
\hline $\begin{array}{l}12 \text { Cardiac disorders MedDRA } \\
\text { Class }\end{array}$ & 4 & 2441 & Risk Ratio (M-H, Random, 95\% CI) & $1.20[0.89,1.61]$ \\
\hline $\begin{array}{l}13 \text { Gastrointestinal disorders } \\
\text { MedDRA Class }\end{array}$ & 6 & 3190 & Risk Ratio (M-H, Random, 95\% CI) & $1.82[1.04,3.19]$ \\
\hline $\begin{array}{l}14 \text { General disorders and } \\
\text { administration site conditions } \\
\text { MedDRA Class }\end{array}$ & 3 & 1256 & Risk Ratio (M-H, Random, 95\% CI) & $1.93[0.32,11.65]$ \\
\hline $\begin{array}{l}15 \text { Neoplasms benign, malignant, } \\
\text { and unspecified MedDRA } \\
\text { Class }\end{array}$ & 6 & 3190 & Risk Ratio (M-H, Random, 95\% CI) & $1.00[0.68,1.46]$ \\
\hline $\begin{array}{l}16 \text { Nervous system disorders } \\
\text { MedDRA Class }\end{array}$ & 6 & 3190 & Risk Ratio (M-H, Random, 95\% CI) & $1.13[0.77,1.68]$ \\
\hline $\begin{array}{l}17 \text { Respiratory, thoracic, and } \\
\text { mediastinal disorders MedDRA } \\
\text { Class }\end{array}$ & 3 & 1256 & Risk Ratio (M-H, Random, 95\% CI) & $0.94[0.42,2.12]$ \\
\hline
\end{tabular}

Comparison 2. Bevacizumab versus ranibizumab, one-year of follow-up, primary outcomes

\begin{tabular}{lcccc} 
Outcome or subgroup title & $\begin{array}{c}\text { No. of } \\
\text { studies }\end{array}$ & $\begin{array}{c}\text { No. of } \\
\text { participants }\end{array}$ & Statistical method & Effect size \\
\hline $\begin{array}{l}1 \text { All-cause death } \\
2 \text { All serious systemic adverse }\end{array}$ & 7 & 3218 & Risk Ratio (M-H, Random, 95\% CI) & $1.23[0.72,2.10]$ \\
events & 8 & 3545 & Risk Ratio (M-H, Random, 95\% CI) & $1.11[0.90,1.37]$ \\
\hline $\begin{array}{l}\text { Systemic safety of bevacizumab versus ranibizumab for neovascular age-related macular degeneration (Review) } \\
\text { Copyright } \odot \text { 2014 The Cochrane Collaboration. Published by John Wiley \& Sons, Ltd. }\end{array}$ & $\mathbf{4 9}$
\end{tabular}




\begin{tabular}{lcccc} 
Outcome or subgroup title & $\begin{array}{c}\text { No. of } \\
\text { studies }\end{array}$ & $\begin{array}{c}\text { No. of } \\
\text { participants }\end{array}$ & Statistical method & Effect size \\
\hline $\begin{array}{l}1 \text { All serious systemic adverse } \\
\text { events }\end{array}$ & 8 & 2480 & Risk Ratio (M-H, Random, 95\% CI) & $1.01[0.82,1.25]$ \\
\hline
\end{tabular}

Comparison 4. Bevacizumab versus ranibizumab, influence analysis: all SSAEs excluding LUCAS

\begin{tabular}{lcccc} 
Outcome or subgroup title & $\begin{array}{c}\text { No. of } \\
\text { studies }\end{array}$ & $\begin{array}{c}\text { No. of } \\
\text { participants }\end{array}$ & Statistical method & Effect size \\
\hline $\begin{array}{l}\text { 1 All serious systemic adverse } \\
\text { events }\end{array}$ & 8 & 3233 & Risk Ratio (M-H, Random, 95\% CI) & $1.19[1.06,1.34]$ \\
\hline
\end{tabular}

Comparison 5. Bevacizumab versus ranibizumab, influence analysis: primary outcomes excluding unpublished studies

\begin{tabular}{lcclc} 
Outcome or subgroup title & $\begin{array}{c}\text { No. of } \\
\text { studies }\end{array}$ & $\begin{array}{c}\text { No. of } \\
\text { participants }\end{array}$ & Statistical method & Effect size \\
\hline 1 All-cause death & 6 & 2745 & Risk Ratio (M-H, Random, 95\% CI) & $1.12[0.78,1.62]$ \\
$\begin{array}{l}\text { All serious systemic adverse } \\
\text { events }\end{array}$ & 6 & 2745 & Risk Ratio (M-H, Random, 95\% CI) & $1.21[1.06,1.37]$ \\
\hline
\end{tabular}

Comparison 6. Bevacizumab versus ranibizumab, secondary analysis: fixed-effect analyses

\begin{tabular}{lccll} 
Outcome or subgroup title & $\begin{array}{c}\text { No. of } \\
\text { studies }\end{array}$ & $\begin{array}{c}\text { No. of } \\
\text { participants }\end{array}$ & Statistical method & Effect size \\
\hline 1 All-cause death & 8 & 3338 & Risk Ratio (M-H, Fixed, 95\% CI) & $1.10[0.78,1.57]$ \\
$\begin{array}{l}\text { 2 All serious systemic adverse } \\
\quad \text { events }\end{array}$ & 9 & 3665 & Risk Ratio (M-H, Fixed, 95\% CI) & $1.12[1.00,1.26]$ \\
3 Arterial thromboembolic event & 6 & 3190 & Risk Ratio (M-H, Fixed, 95\% CI) & $0.90[0.62,1.32]$ \\
4 Gastrointestinal perforation & 1 & 161 & Risk Ratio (M-H, Fixed, 95\% CI) & $0.0[0.0,0.0]$ \\
5 Infections & 6 & 3190 & Risk Ratio (M-H, Fixed, 95\% CI) & $1.35[0.97,1.87]$ \\
6 Myocardial infarction & 6 & 3190 & Risk Ratio (M-H, Fixed, 95\% CI) & $0.71[0.37,1.35]$ \\
7 Neutropenia & 1 & 161 & Risk Ratio (M-H, Fixed, 95\% CI) & $0.0[0.0,0.0]$ \\
8 Non-ocular haemorrhage & 3 & 1256 & Risk Ratio (M-H, Fixed, 95\% CI) & $0.36[0.08,1.50]$ \\
9 Stroke & 6 & 3190 & Risk Ratio (M-H, Fixed, 95\% CI) & $0.83[0.42,1.63]$ \\
10 Treatment-related drug & 0 & 0 & Risk Ratio (M-H, Fixed, 95\% CI) & $0.0[0.0,0.0]$ \\
$\quad$ discontinuation & & & &
\end{tabular}

Systemic safety of bevacizumab versus ranibizumab for neovascular age-related macular degeneration (Review) 
11 Vascular events associated with anti-VEGF treatment

12 Cardiac disorders MedDRA Class

13 Gastrointestinal disorders MedDRA Class

14 General disorders and administration site conditions MedDRA Class

15 Neoplasms benign, malignant, and unspecified MedDRA Class

16 Nervous system disorders MedDRA Class

17 Respiratory, thoracic, and mediastinal disorders MedDRA Class
$1.15[0.85,1.56]$

$1.19[0.88,1.59]$

$1.93[1.20,3.11]$

$1.39[0.73,2.65]$

$0.94[0.42,2.12]$

\section{Analysis I.I. Comparison I Bevacizumab versus ranibizumab, longest follow-up, Outcome I All-cause}

death.

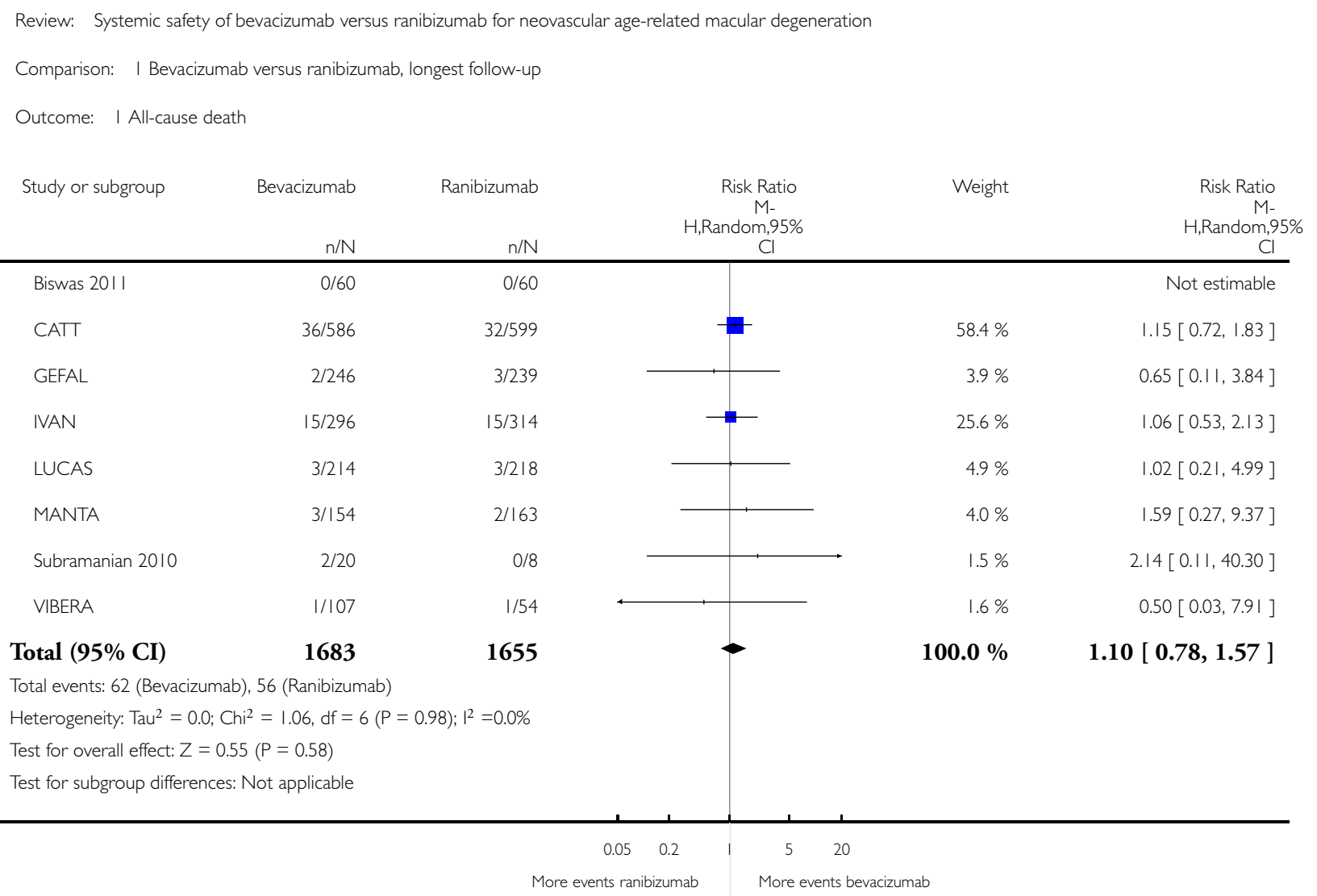




\section{Analysis I.2. Comparison I Bevacizumab versus ranibizumab, longest follow-up, Outcome 2 All serious}

systemic adverse events.

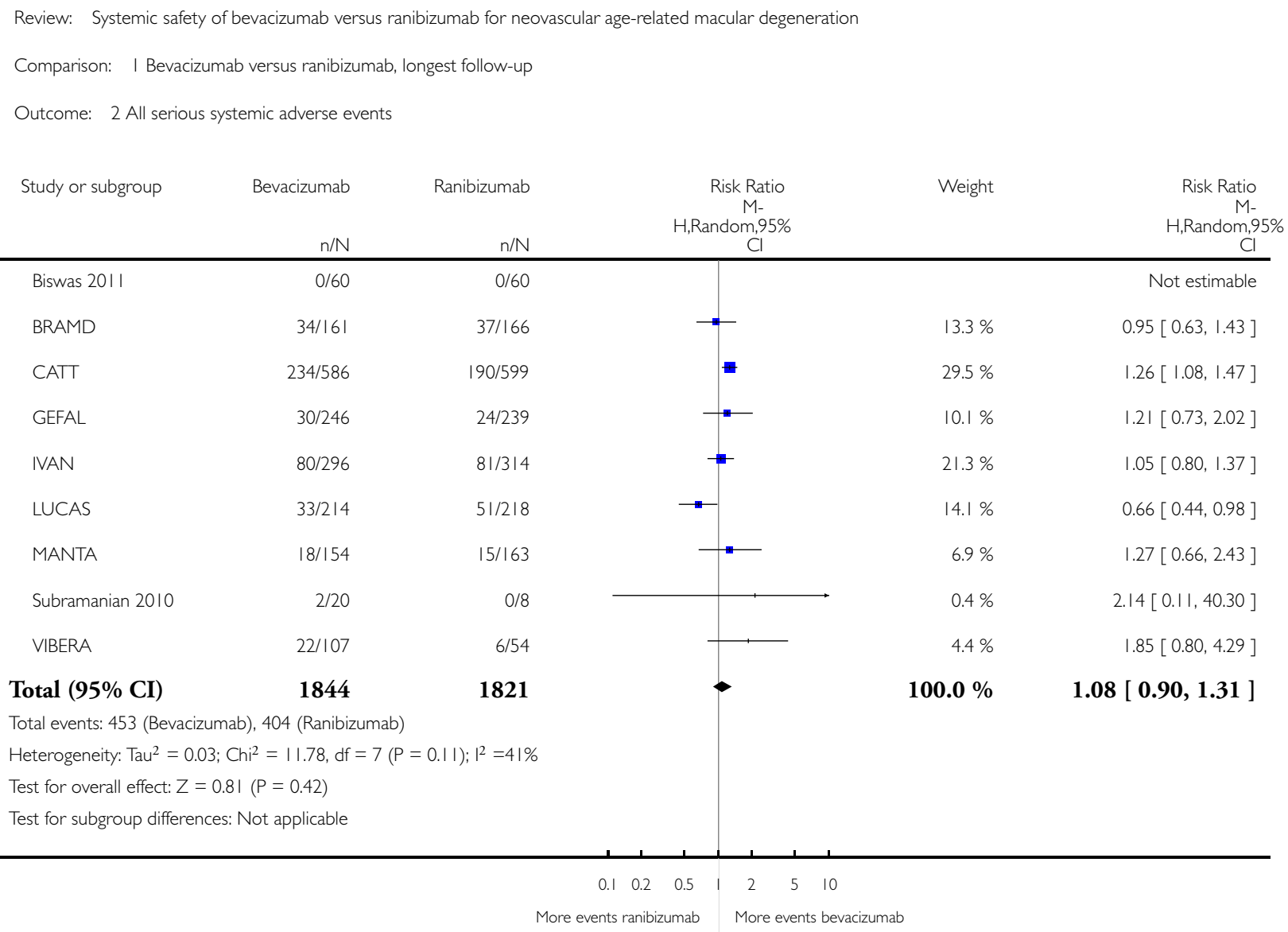


Analysis I.3. Comparison I Bevacizumab versus ranibizumab, longest follow-up, Outcome 3 Arterial thromboembolic event.

\begin{tabular}{|c|c|c|c|c|c|}
\hline \multicolumn{6}{|c|}{ Comparison: I Bevacizumab versus ranibizumab, longest follow-up } \\
\hline \multicolumn{6}{|c|}{ Outcome: 3 Arterial thromboembolic event } \\
\hline Study or subgroup & Bevacizumab & $\begin{array}{r}\text { Ranibizumab } \\
n / N \\
\end{array}$ & $\begin{array}{c}\text { Risk Ratio } \\
\text { M- } \\
\text { H,Random,95\% } \\
\mathrm{Cl} \\
\end{array}$ & Weight & $\begin{array}{c}\text { Risk Ratio } \\
\text { M- } \\
\text { H,Random,95\% } \\
\text { Cl } \\
\end{array}$ \\
\hline CATT & $29 / 586$ & $28 / 599$ & 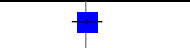 & $57.2 \%$ & $1.06[0.64,1.76]$ \\
\hline GEFAL & 1/246 & 1/239 & 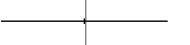 & $2.1 \%$ & $0.97[0.06,15.44]$ \\
\hline IVAN & $10 / 296$ & $13 / 314$ & $\rightarrow-$ & $23.4 \%$ & $0.82[0.36,1.83]$ \\
\hline LUCAS & $3 / 214$ & $10 / 218$ & $\longrightarrow$ & $9.6 \%$ & $0.31[0.09,1.10]$ \\
\hline MANTA & $5 / 154$ & $3 / 163$ & $\longrightarrow$ & $7.8 \%$ & $1.76[0.43,7.26]$ \\
\hline VIBERA & 0/107 & $0 / 54$ & & & Not estimable \\
\hline Total $(95 \% \mathrm{CI})$ & 1603 & 1587 & 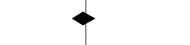 & $100.0 \%$ & $0.92[0.62,1.37]$ \\
\hline \multicolumn{6}{|c|}{ Total events: 48 (Bevacizumab), 55 (Ranibizumab) } \\
\hline \multicolumn{6}{|c|}{ Heterogeneity: $\mathrm{Tau}^{2}=0.00 ; \mathrm{Ch}^{2}=4.07, \mathrm{df}=4(\mathrm{P}=0.40) ; \mathrm{I}^{2}=2 \%$} \\
\hline \multicolumn{6}{|c|}{ Test for overall effect: $Z=0.42(P=0.68)$} \\
\hline \multicolumn{6}{|c|}{ Test for subgroup differences: Not applicable } \\
\hline
\end{tabular}




\section{Analysis I.4. Comparison I Bevacizumab versus ranibizumab, longest follow-up, Outcome 4}

Gastrointestinal perforation.

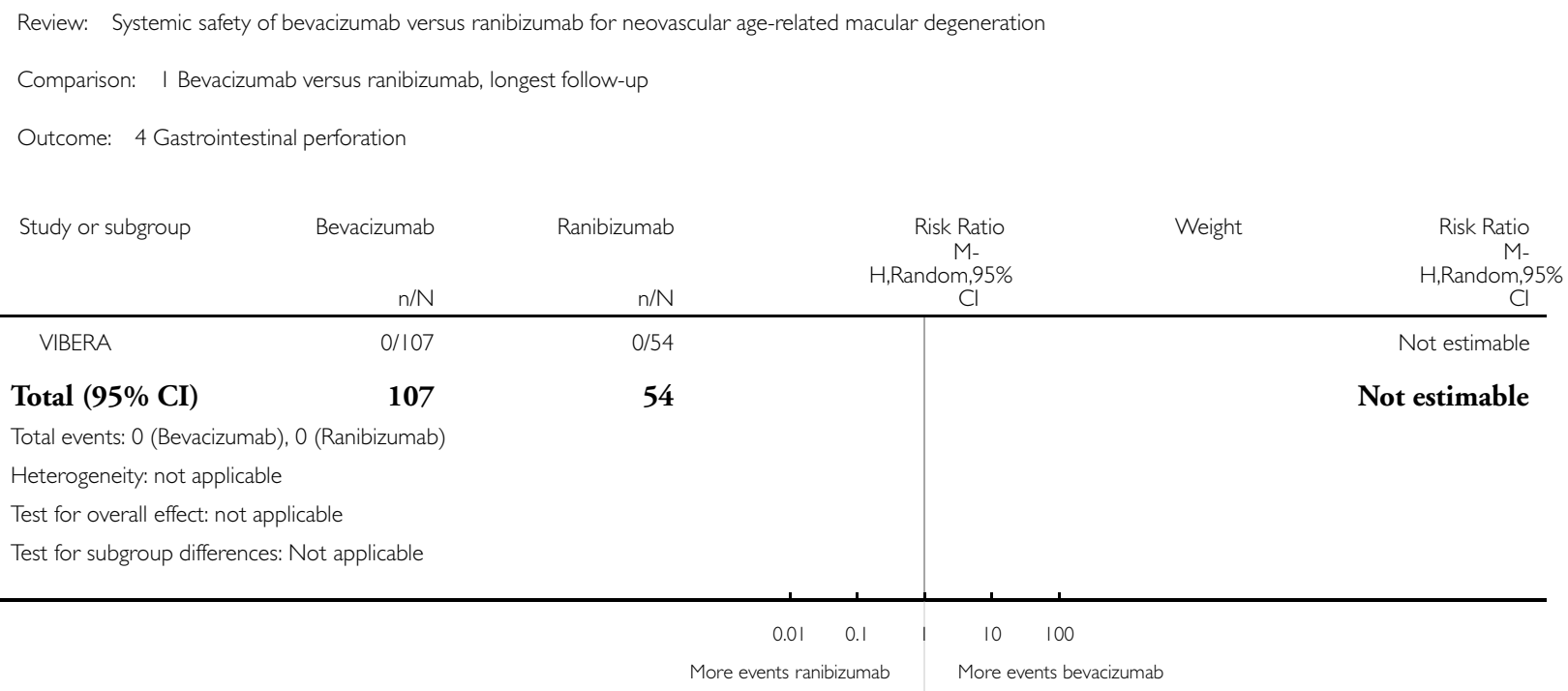




\section{Analysis I.5. Comparison I Bevacizumab versus ranibizumab, longest follow-up, Outcome 5 Infection.}

Review: Systemic safety of bevacizumab versus ranibizumab for neovascular age-related macular degeneration

Comparison: I Bevacizumab versus ranibizumab, longest follow-up

Outcome: 5 Infection

Study or subgroup

Bevacizumab Ranibizumab

Risk Ratio

M-

$\mathrm{H}, \mathrm{Random}, 95 \%$

Weight

Risk Ratio

$\mathrm{M}$ -

$\begin{array}{rrr} & n / N & n / N\end{array}$

C

$\begin{array}{ll}\text { GEFAL } & \text { 4/246 }\end{array}$

\begin{tabular}{l|l} 
IVAN & 12/296
\end{tabular}

LUCAS

$3 / 214$

$4 / 218$

MANTA

$3 / 154$

$3 / 163$

VIBERA

$3 / 107$

$0 / 54$

Total (95\% CI)

1603

1587

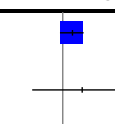

$70.9 \%$

Cl

Total events: 79 (Bevacizumab), 59 (Ranibizumab)

Heterogeneity: $\mathrm{Tau}^{2}=0.0 ; \mathrm{Chi}^{2}=1.26, \mathrm{df}=5(\mathrm{P}=0.94) ; \mathrm{I}^{2}=0.0 \%$

Test for overall effect: $Z=1.75(P=0.080)$

Test for subgroup differences: Not applicable

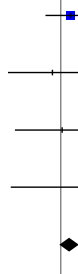

$3.8 \%$

$14.9 \%$

$4.9 \%$

$4.3 \%$

$1.2 \%$

$100.0 \%$

$1.34[0.97,1.86$ ]

\section{$0.01 \quad 0.1$ \\ 10 \\ 100 \\ More events ranibizumab \\ More events bevacizumab}


Analysis I.6. Comparison I Bevacizumab versus ranibizumab, longest follow-up, Outcome 6 Myocardial infarction.

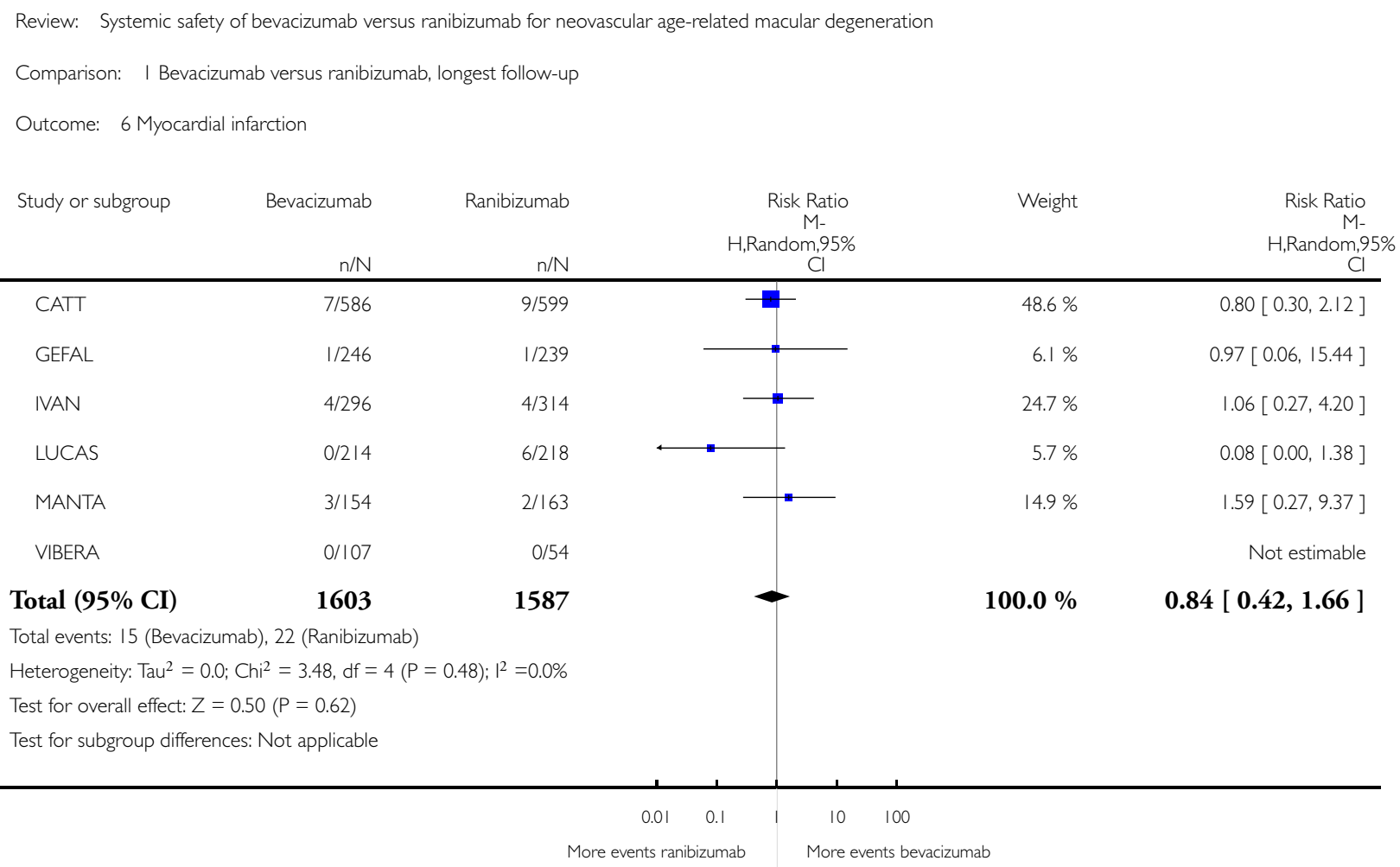




\section{Analysis I.7. Comparison I Bevacizumab versus ranibizumab, longest follow-up, Outcome 7 Neutropenia.}

Review: Systemic safety of bevacizumab versus ranibizumab for neovascular age-related macular degeneration

Comparison: I Bevacizumab versus ranibizumab, longest follow-up

Outcome: 7 Neutropenia

Study or subgroup $\quad$ Bevacizumab $\quad$ Ranibizumab

\begin{tabular}{lcc} 
& $\mathrm{n} / \mathrm{N}$ & $\mathrm{n} / \mathrm{N}$ \\
\hline VIBERA & $0 / 107$ & $0 / 54$ \\
& $\mathbf{1 0 7}$ & $\mathbf{5}$
\end{tabular}

Total (95\% CI) 107

54

\begin{tabular}{|c|c|c|}
\hline $\begin{array}{r}\text { Risk Ratio } \\
\text { M- } \\
\text { H,Random,95\% } \\
\text { Cl }\end{array}$ & Weight & $\begin{array}{c}\text { Risk Ratio } \\
\text { M- } \\
\text { H,Random,95\% } \\
\text { Cl }\end{array}$ \\
\hline & & Not estimable \\
\hline & & t estimable \\
\hline
\end{tabular}

Total events: 0 (Bevacizumab), 0 (Ranibizumab)

Heterogeneity: not applicable

Test for overall effect: not applicable

Test for subgroup differences: Not applicable

\section{Analysis I.8. Comparison I Bevacizumab versus ranibizumab, longest follow-up, Outcome 8 Non-ocular} haemorrhage.

Review: Systemic safety of bevacizumab versus ranibizumab for neovascular age-related macular degeneration

Comparison: I Bevacizumab versus ranibizumab, longest follow-up

Outcome: 8 Non-ocular haemorrhage

\begin{tabular}{|c|c|c|c|c|c|}
\hline Study or subgroup & Bevacizumab & Ranibizumab & $\begin{array}{c}\text { Risk Ratio } \\
\text { M- } \\
\text { H,Random,95\% } \\
\text { Cl }\end{array}$ & Weight & $\begin{array}{c}\text { Risk Ratio } \\
\text { M- } \\
\text { H,Random,95\% } \\
\text { Cl }\end{array}$ \\
\hline GEFAL & $0 / 246$ & $3 / 239$ & $\begin{array}{l}-1 \\
-\end{array}$ & $27.9 \%$ & $0.14[0.01,2.67]$ \\
\hline IVAN & $1 / 296$ & $3 / 314$ & 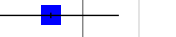 & $48.0 \%$ & $0.35[0.04,3.38]$ \\
\hline VIBERA & $1 / 107$ & $0 / 54$ & & $24.1 \%$ & $1.53[0.06,36.89]$ \\
\hline
\end{tabular}

Total $(95 \% \mathrm{CI})$

649

607

$100.0 \%$

$0.39[0.08,1.85]$

Total events: 2 (Bevacizumab), 6 (Ranibizumab)

Heterogeneity: $\operatorname{Tau}^{2}=0.0 ; \mathrm{Chi}^{2}=1.19, \mathrm{df}=2(\mathrm{P}=0.55) ; \mathrm{I}^{2}=0.0 \%$

Test for overall effect: $Z=1.19(P=0.23)$

Test for subgroup differences: Not applicable

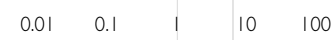

More events ranibizumab More events bevacizumab 


\section{Analysis I.9. Comparison I Bevacizumab versus ranibizumab, longest follow-up, Outcome 9 Stroke.}

Review: Systemic safety of bevacizumab versus ranibizumab for neovascular age-related macular degeneration

Comparison: I Bevacizumab versus ranibizumab, longest follow-up

Outcome: 9 Stroke

Bevacizumab Ranibizumab

Risk Ratio

M-

Weight

Risk Ratio

H,Random, $95 \%$

\begin{tabular}{|c|c|c|c|c|c|}
\hline & $\mathrm{n} / \mathrm{N}$ & $\mathrm{n} / \mathrm{N}$ & $\begin{array}{c}\mathrm{H}, \mathrm{Random}, 95 \% \\
\mathrm{Cl}\end{array}$ & & $\begin{array}{r}\mathrm{H}, \text { Random,9! } \\
\mathrm{Cl} \\
\end{array}$ \\
\hline CATT & $8 / 586$ & $8 / 599$ & 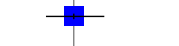 & $49.6 \%$ & $1.02[0.39,2.7 \mid]$ \\
\hline GEFAL & $0 / 246$ & $0 / 239$ & & & Not estimable \\
\hline IVAN & $3 / 296$ & $6 / 314$ & $\longrightarrow$ & $24.8 \%$ & $0.53[0.13,2.10]$ \\
\hline LUCAS & $2 / 214$ & $3 / 218$ & & $14.8 \%$ & $0.68[0.11,4.02]$ \\
\hline MANTA & $1 / 154$ & $1 / 163$ & & $6.2 \%$ & $1.06[0.07,16.77]$ \\
\hline VIBERA & $1 / 107$ & $0 / 54$ & & $4.6 \%$ & $1.53[0.06,36.89]$ \\
\hline Total (95\% CI) & 1603 & 1587 & & $100.0 \%$ & $0.83[0.42,1.66]$ \\
\hline \multicolumn{6}{|c|}{ Total events: 15 (Bevacizumab), I8 (Ranibizumab) } \\
\hline \multicolumn{6}{|c|}{ Heterogeneity: $\operatorname{Tau}^{2}=0.0 ; \mathrm{Chi}^{2}=0.80, \mathrm{df}=4(\mathrm{P}=0.94) ;\left.\right|^{2}=0.0 \%$} \\
\hline \multicolumn{6}{|c|}{ Test for overall effect: $Z=0.52(P=0.61)$} \\
\hline Test for subgroup diff & pplicable & & & & \\
\hline
\end{tabular}

$\begin{array}{lllll}0.01 & 0.1 & 1 & 10 & 100\end{array}$

More events ranibizumab More events bevacizumab 
Analysis I.II. Comparison I Bevacizumab versus ranibizumab, longest follow-up, Outcome II Vascular events associated with anti-VEGF treatment.

Review: Systemic safety of bevacizumab versus ranibizumab for neovascular age-related macular degeneration

Comparison: I Bevacizumab versus ranibizumab, longest follow-up

Outcome: I Vascular events associated with anti-VEGF treatment

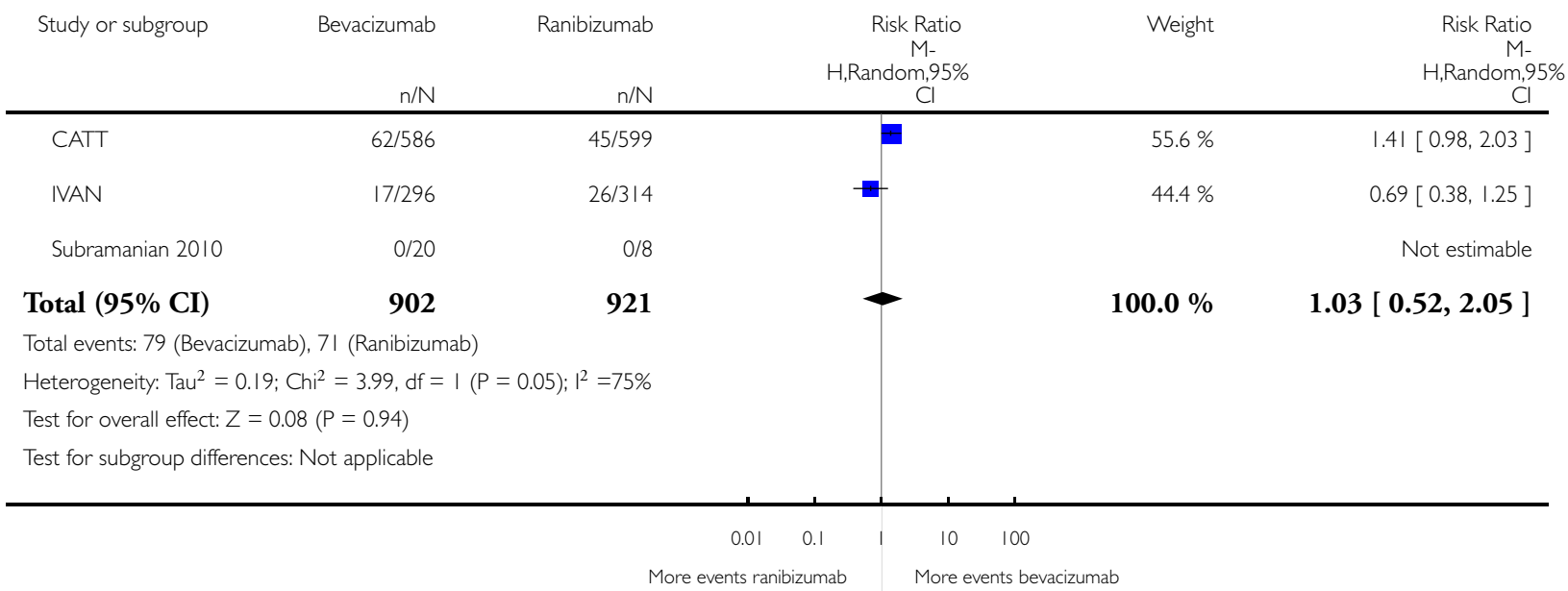




\section{Analysis I.12. Comparison I Bevacizumab versus ranibizumab, longest follow-up, Outcome I2 Cardiac}

disorders MedDRA Class.

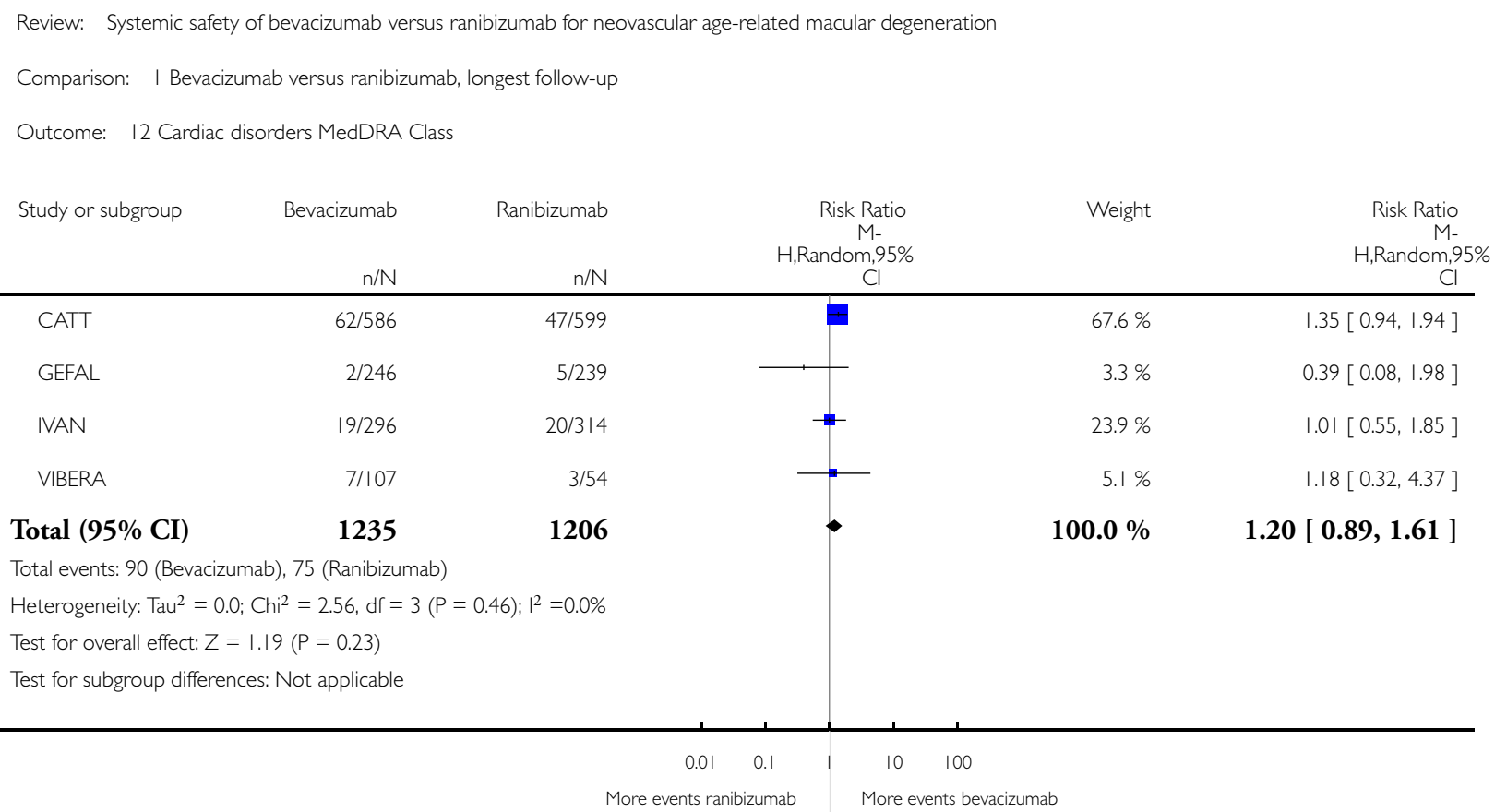


Analysis I.13. Comparison I Bevacizumab versus ranibizumab, longest follow-up, Outcome 13 Gastrointestinal disorders MedDRA Class.

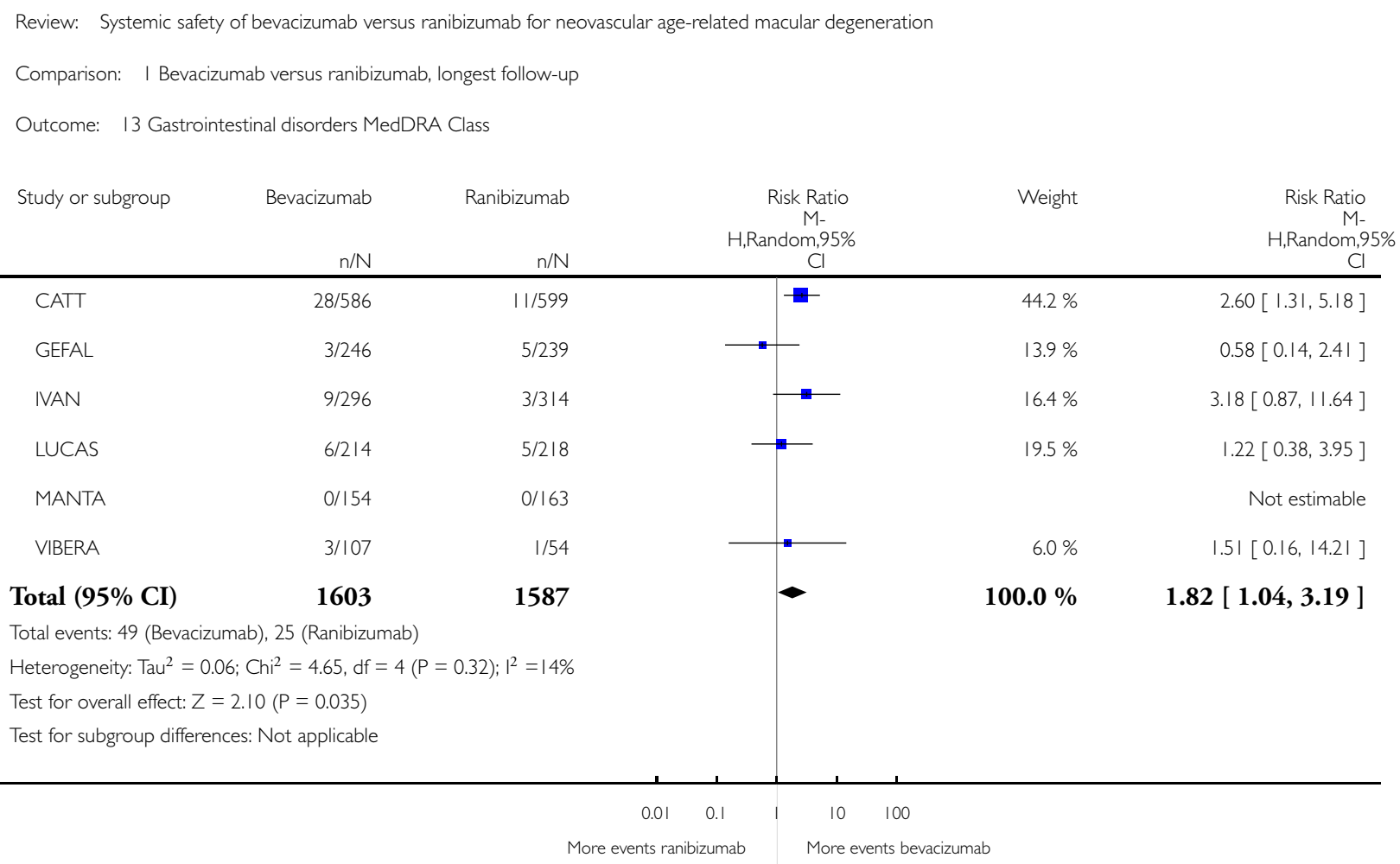


Analysis I.I4. Comparison I Bevacizumab versus ranibizumab, longest follow-up, Outcome I4 General disorders and administration site conditions MedDRA Class.

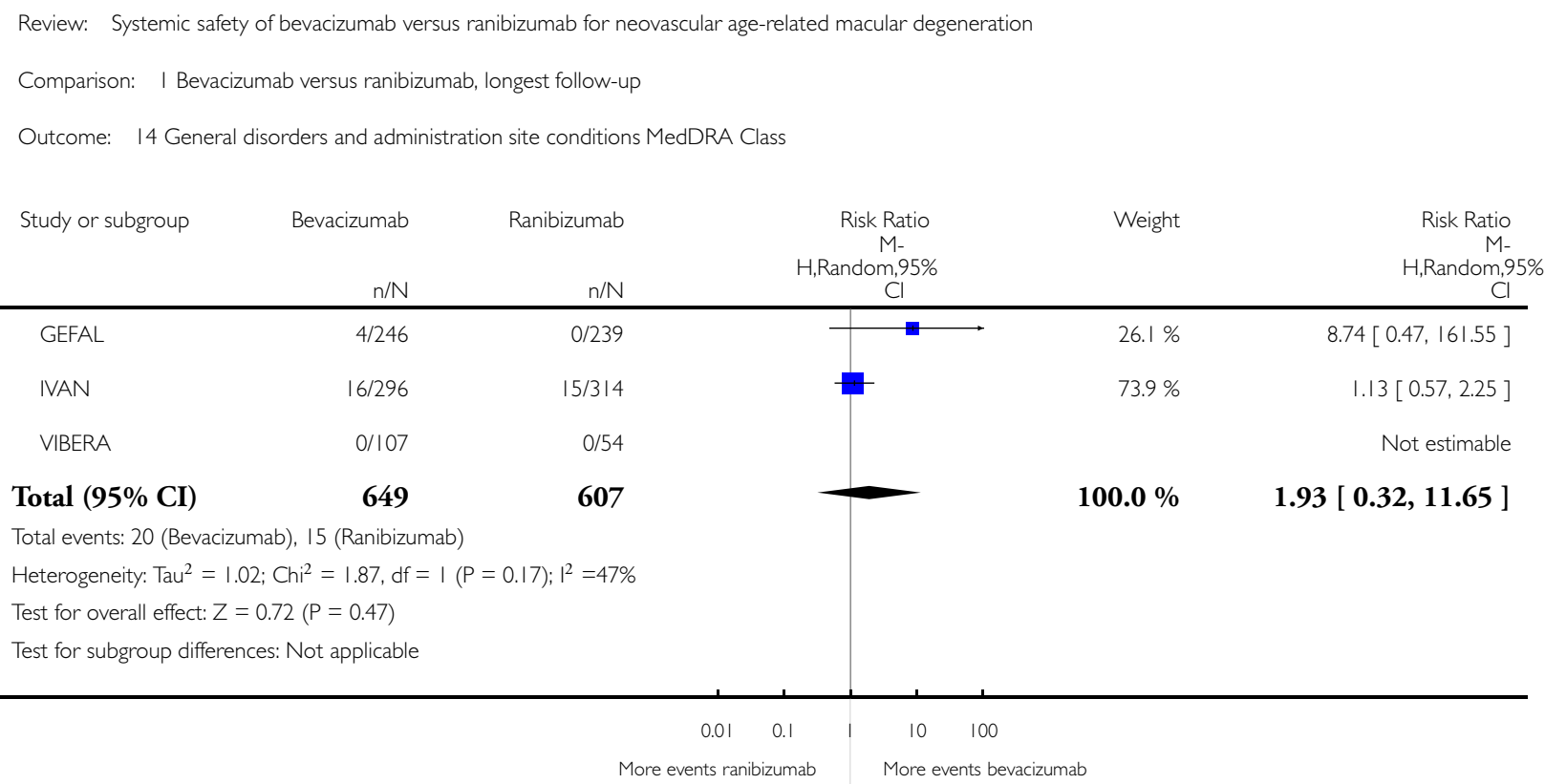


Analysis I.I5. Comparison I Bevacizumab versus ranibizumab, longest follow-up, Outcome I5 Neoplasms benign, malignant, and unspecified MedDRA Class.

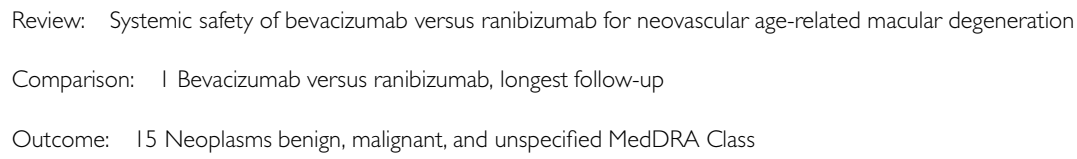

1587

9

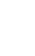

$\prod_{-}$

$24.6 \%$

$17.8 \%$

$2.6 \%$

$97[0.06,15.44]$

Total events: 5 I (Bevacizumab), 5 I (Ranibizumab)

Heterogeneity: Tau $^{2}=0.0 ; \mathrm{Chi}^{2}=1.64, \mathrm{df}=5(\mathrm{P}=0.90) ; \mathrm{I}^{2}=0.0 \%$

Test for overall effect: $Z=0.01(P=0.99)$

Test for subgroup differences: Not applicable

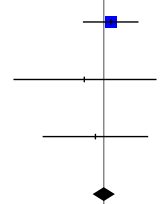

$4.8 \%$

$1.35[0.62,2.93]$

$1.27[0.51,3.16]$

54

$100.0 \%$

$0.53[0.05,5.78]$

$0.76[0.13,4.40]$ 
Analysis I.16. Comparison I Bevacizumab versus ranibizumab, longest follow-up, Outcome 16 Nervous system disorders MedDRA Class.

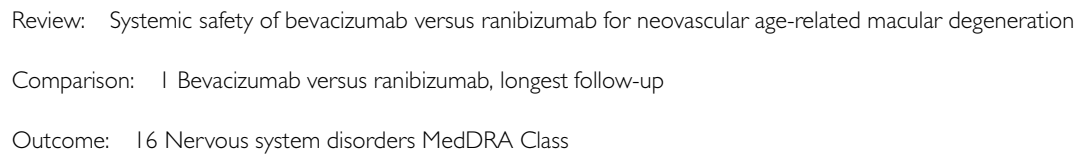

CATT 36/586 34/599

$\mathrm{n} / \mathrm{N}$

$3 / 246$

$0 / 239$

GEFAL

$8 / 296$

$9 / 314$

IVAN

$1 / 214$

$0 / 218$

LUCAS

$2 / 154$

$1 / 163$

MANTA

1/107

$0 / 54$

1603

1587

$74.9 \%$

Total (95\% CI)

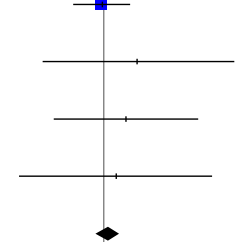

$100.0 \%$

Total events: 5 I (Bevacizumab), 44 (Ranibizumab)

Heterogeneity: $\operatorname{Tau}^{2}=0.0 ; \mathrm{Chi}^{2}=2.29, \mathrm{df}=5(\mathrm{P}=0.8 \mathrm{I}) ; \mathrm{I}^{2}=0.0 \%$

Test for overall effect: $Z=0.63(P=0.53)$

Test for subgroup differences: Not applicable

$1.8 \%$

$17.6 \%$

$1.5 \%$

$2.7 \%$

$1.5 \%$

\begin{tabular}{cr} 
Weight & $\begin{array}{r}\text { Risk Ratio } \\
\mathrm{M}- \\
\mathrm{H}, \text { Random,95\% } \\
\mathrm{Cl}\end{array}$ \\
\hline $74.9 \%$ & $1.08[0.69,1.71]$ \\
$1.8 \%$ & $6.80[0.35,130.98]$ \\
$17.6 \%$ & $0.94[0.37,2.41]$ \\
$1.5 \%$ & $3.06[0.13,74.60]$ \\
$2.7 \%$ & $2.12[0.19,23.11]$ \\
$1.5 \%$ & $1.53[0.06,36.89]$
\end{tabular}

1.13 [ $0.77,1.68$ ]

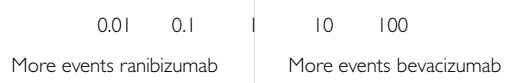


Analysis I.I7. Comparison I Bevacizumab versus ranibizumab, longest follow-up, Outcome I7 Respiratory, thoracic, and mediastinal disorders MedDRA Class.

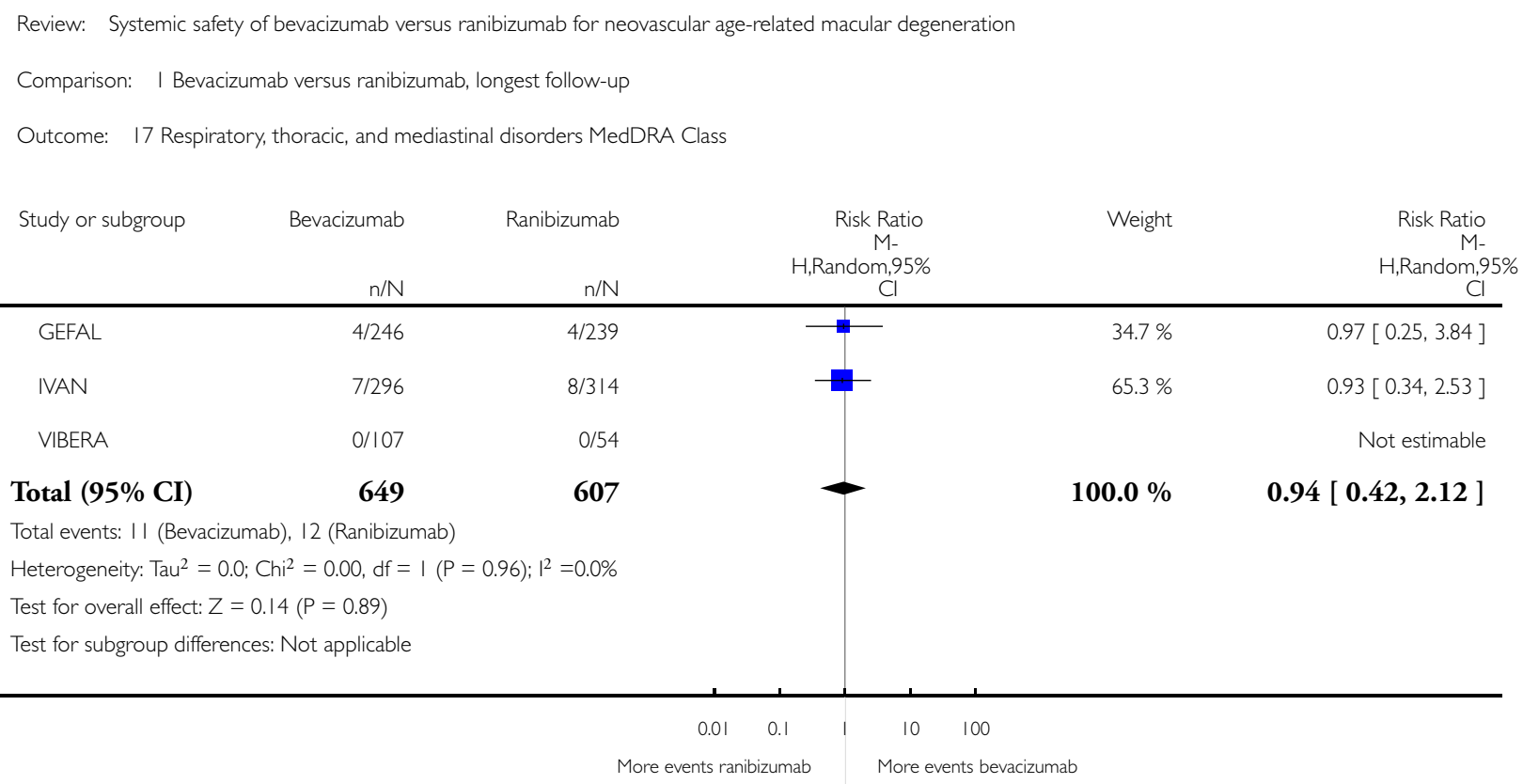


Analysis 2.I. Comparison 2 Bevacizumab versus ranibizumab, one-year of follow-up, primary outcomes, Outcome I All-cause death.

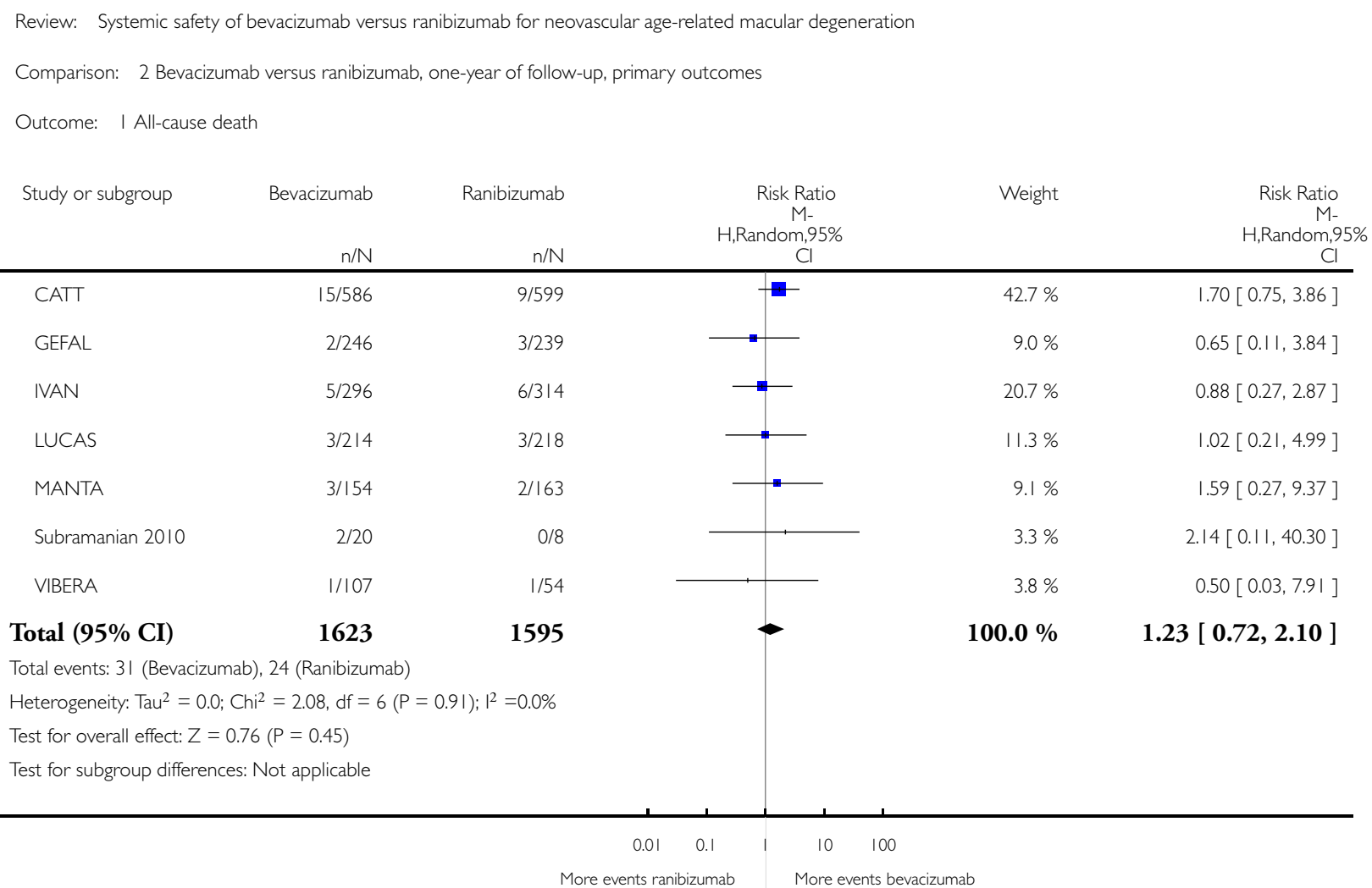


Analysis 2.2. Comparison 2 Bevacizumab versus ranibizumab, one-year of follow-up, primary outcomes, Outcome 2 All serious systemic adverse events.

Review: Systemic safety of bevacizumab versus ranibizumab for neovascular age-related macular degeneration

Comparison: 2 Bevacizumab versus ranibizumab, one-year of follow-up, primary outcomes

Outcome: 2 All serious systemic adverse events

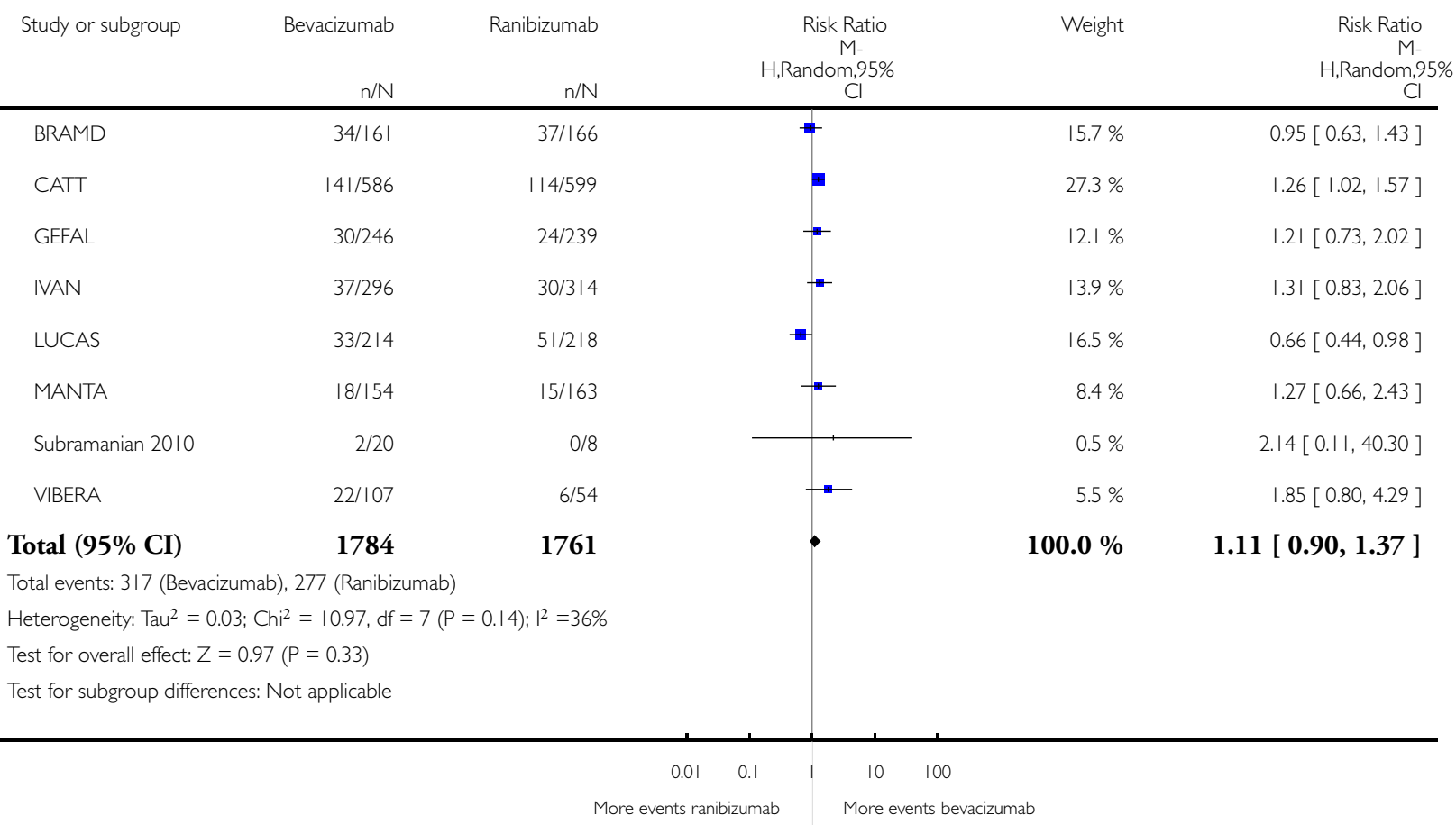


Analysis 3.I. Comparison 3 Bevacizumab versus ranibizumab, influence analysis: all SSAEs excluding CATT, Outcome I All serious systemic adverse events.

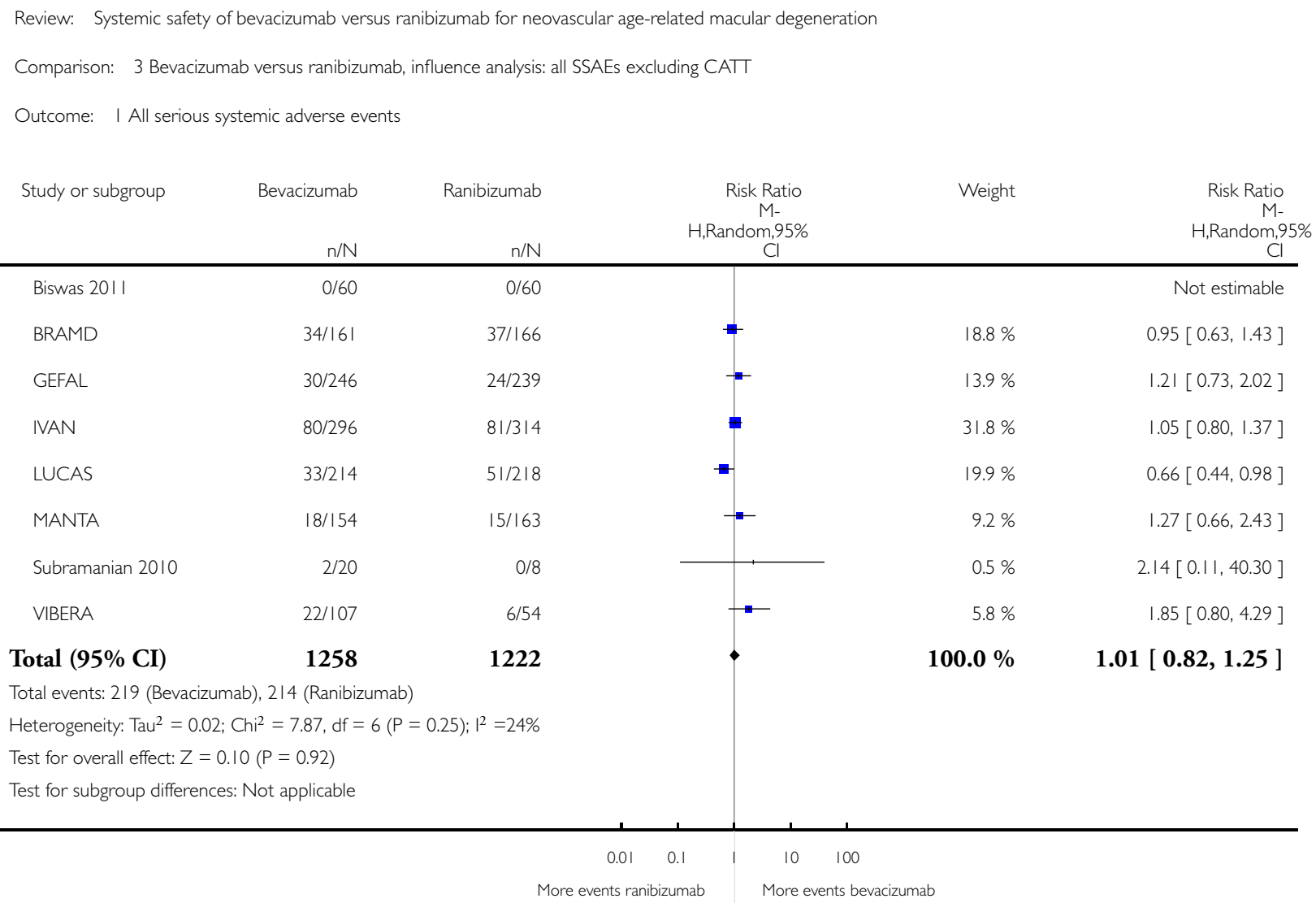


Analysis 4.I. Comparison 4 Bevacizumab versus ranibizumab, influence analysis: all SSAEs excluding LUCAS, Outcome I All serious systemic adverse events.

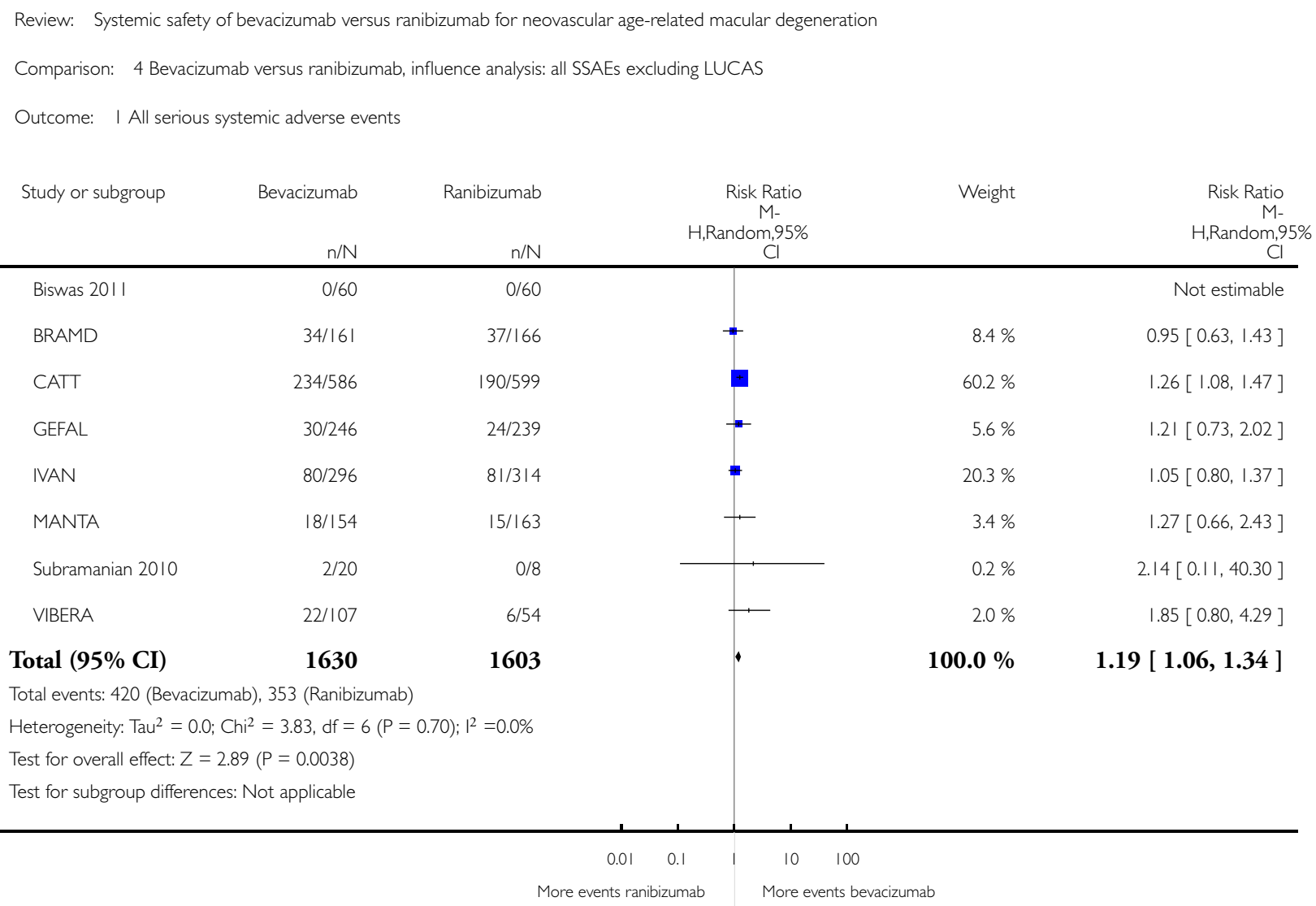


Analysis 5.I. Comparison 5 Bevacizumab versus ranibizumab, influence analysis: primary outcomes excluding unpublished studies, Outcome I All-cause death.

Review: Systemic safety of bevacizumab versus ranibizumab for neovascular age-related macular degeneration

Comparison: 5 Bevacizumab versus ranibizumab, influence analysis: primary outcomes excluding unpublished studies

Outcome: I All-cause death

Study or subgroup

Bevacizumab

Ranibizumab

Risk Ratio

$\mathrm{M}$ -

$\mathrm{H}$,Random,95\%

Weight

Risk Ratio

Cl

Biswas 201।

$\frac{n / N}{0 / 60}$

$\mathrm{n} / \mathrm{N}$

0/60

$32 / 599$

3/239

GEFAL

$36 / 586$

$15 / 314$

IVAN

15/296

$2 / 163$

MANTA

$3 / 154$

$0 / 8$

$2 / 20$

$0 / 8$

1362

1383

Total (95\% CI)

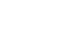

Total events: 58 (Bevacizumab), 52 (Ranibizumab)

Heterogeneity: $\mathrm{Tau}^{2}=0.0 ; \mathrm{Chi}^{2}=0.74, \mathrm{df}=4(\mathrm{P}=0.95) ; \mathrm{I}^{2}=0.0 \%$

Test for overall effect: $Z=0.63(P=0.53)$

Test for subgroup differences: Not applicable

\begin{tabular}{rrr} 
& & $\begin{array}{c}H, \text { Random,95\% } \\
\text { Not estimable }\end{array}$ \\
\hline & $62.5 \%$ & $1.15[0.72,1.83]$ \\
& $4.2 \%$ & $0.65[0.11,3.84]$ \\
& $27.5 \%$ & $1.06[0.53,2.13]$ \\
& $4.2 \%$ & $1.59[0.27,9.37]$ \\
1. & $1.6 \%$ & $2.14[0.11,40.30]$
\end{tabular}

$\begin{array}{ccccc}0.01 & 0.1 & 1 & 10 & 100 \\ \text { More events ranibizumab } & & \text { More events bevacizumab }\end{array}$ 
Analysis 5.2. Comparison 5 Bevacizumab versus ranibizumab, influence analysis: primary outcomes excluding unpublished studies, Outcome 2 All serious systemic adverse events.

Review: Systemic safety of bevacizumab versus ranibizumab for neovascular age-related macular degeneration

Comparison: 5 Bevacizumab versus ranibizumab, influence analysis: primary outcomes excluding unpublished studies

Outcome: 2 All serious systemic adverse events

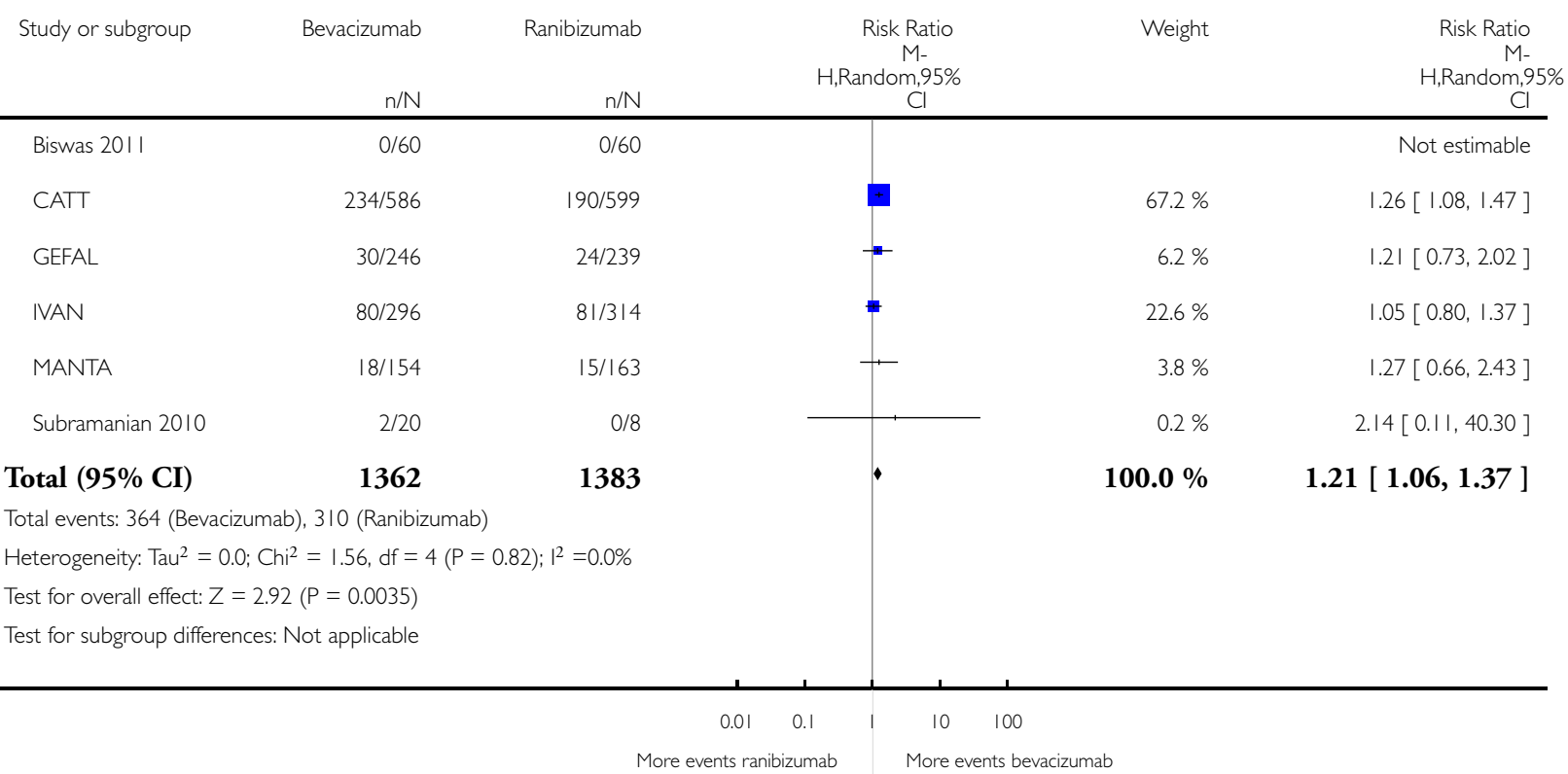


Analysis 6.I. Comparison 6 Bevacizumab versus ranibizumab, secondary analysis: fixed-effect analyses, Outcome I All-cause death.

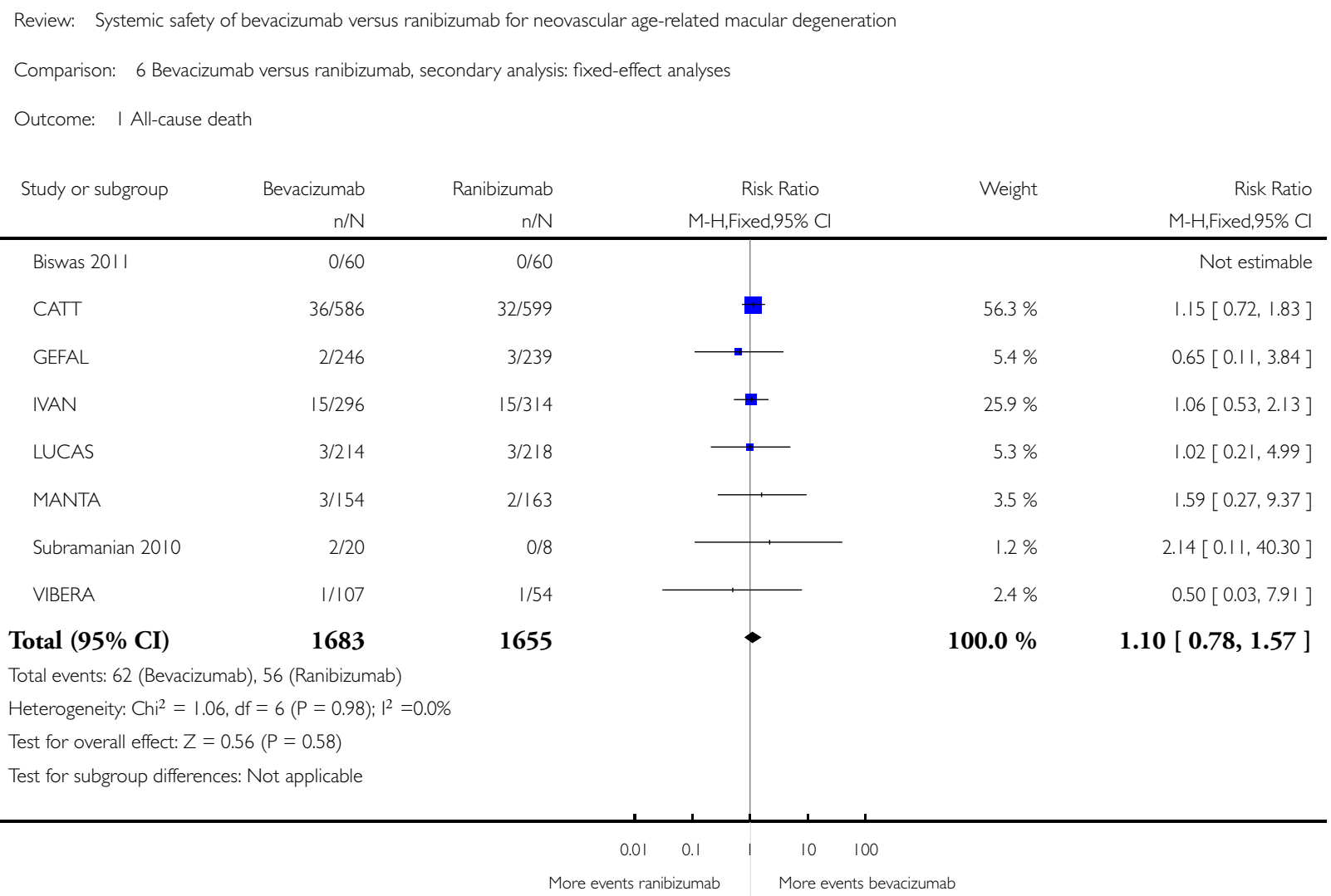




\section{Analysis 6.2. Comparison 6 Bevacizumab versus ranibizumab, secondary analysis: fixed-effect analyses,}

Outcome 2 All serious systemic adverse events.

\begin{tabular}{|c|c|c|c|c|c|}
\hline \multicolumn{6}{|c|}{ Comparison: 6 Bevacizumab versus ranibizumab, secondary analysis: fixed-effect analyses } \\
\hline \multicolumn{6}{|c|}{ Outcome: 2 All serious systemic adverse events } \\
\hline \multirow[t]{2}{*}{ Study or subgroup } & Bevacizumab & Ranibizumab & Risk Ratio & Weight & Risk Ratio \\
\hline & $\mathrm{n} / \mathrm{N}$ & $\mathrm{n} / \mathrm{N}$ & \multicolumn{2}{|l|}{ M-H,Fixed,95\% Cl } & M-H,Fixed,95\% Cl \\
\hline Biswas 2011 & $0 / 60$ & $0 / 60$ & & & Not estimable \\
\hline BRAMD & $34 / 161$ & $37 / 166$ & $\rightarrow$ & $9.1 \%$ & $0.95[0.63,1.43]$ \\
\hline CAT & $234 / 586$ & 190/599 & $\mathbf{\theta}$ & $46.9 \%$ & $1.26[1.08,1.47]$ \\
\hline GEFAL & $30 / 246$ & $24 / 239$ & $\rightarrow$ & $6.1 \%$ & $1.21[0.73,2.02]$ \\
\hline IVAN & $80 / 296$ & $81 / 314$ & \# & $19.6 \%$ & $1.05[0.80,1.37]$ \\
\hline LUCAS & $33 / 214$ & $51 / 218$ & $\rightarrow$ & $12.6 \%$ & $0.66[0.44,0.98]$ \\
\hline MANTA & $18 / 154$ & $15 / 163$ & + & $3.6 \%$ & $1.27[0.66,2.43]$ \\
\hline Subramanian 2010 & $2 / 20$ & $0 / 8$ & 2 & $0.2 \%$ & $2.14[0.11,40.30]$ \\
\hline VIBERA & $22 / 107$ & $6 / 54$ & — & $2.0 \%$ & $1.85[0.80,4.29]$ \\
\hline Total $(95 \% \mathrm{CI})$ & 1844 & 1821 & - & $100.0 \%$ & $1.12[1.00,1.26]$ \\
\hline \multicolumn{6}{|c|}{ Total events: 453 (Bevacizumab), 404 (Ranibizumab) } \\
\hline \multicolumn{6}{|c|}{ Heterogeneity: $\mathrm{Chi}^{2}=11.78, \mathrm{df}=7(\mathrm{P}=0.11) ; \mathrm{I}^{2}=41 \%$} \\
\hline \multicolumn{6}{|c|}{ Test for overall effect: $Z=2.01 \quad(P=0.044)$} \\
\hline Test for subgroup diffe & Vot applicable & & & & \\
\hline
\end{tabular}


Analysis 6.3. Comparison 6 Bevacizumab versus ranibizumab, secondary analysis: fixed-effect analyses, Outcome 3 Arterial thromboembolic event.

Review: Systemic safety of bevacizumab versus ranibizumab for neovascular age-related macular degeneration

Comparison: 6 Bevacizumab versus ranibizumab, secondary analysis: fixed-effect analyses

Outcome: 3 Arterial thromboembolic event

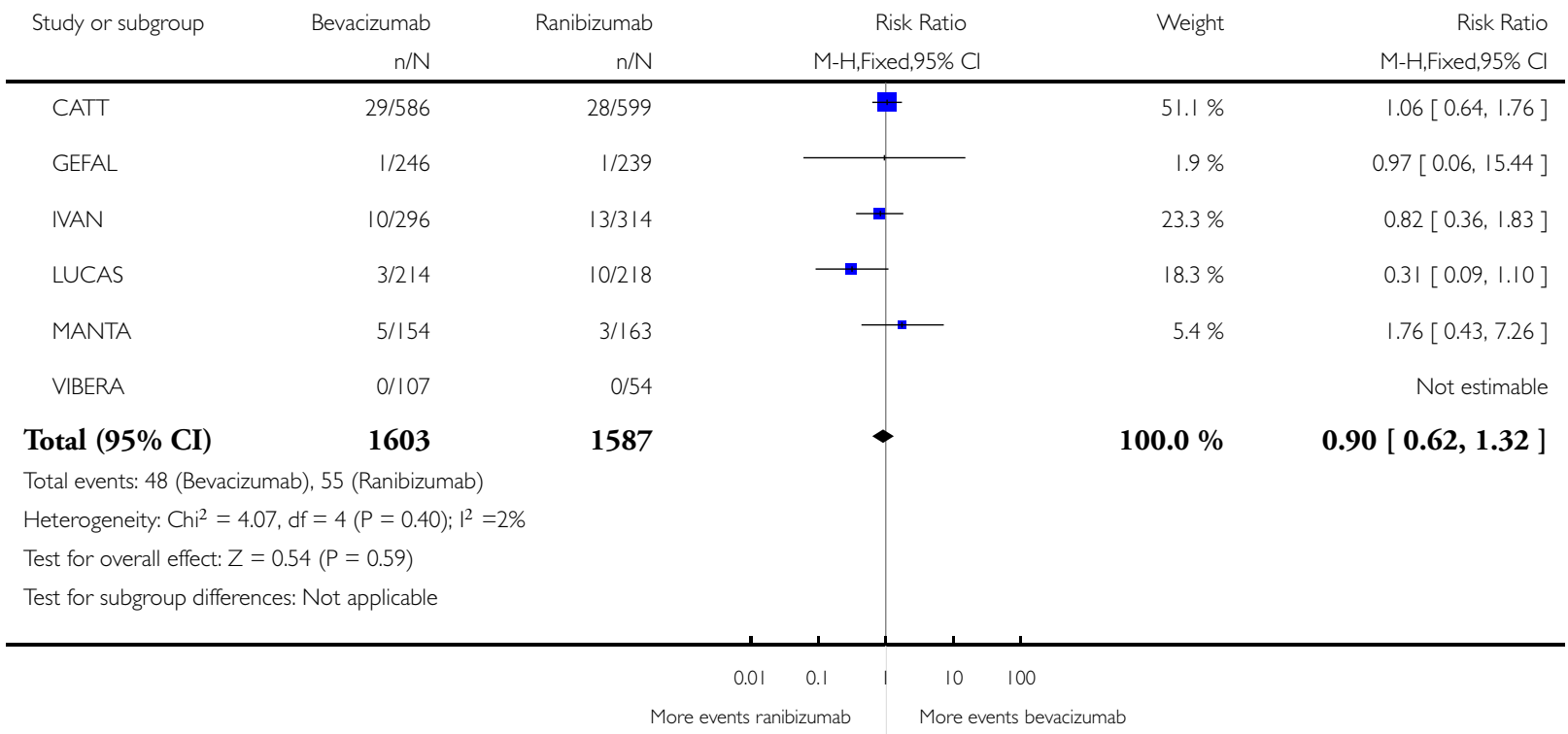


Analysis 6.4. Comparison 6 Bevacizumab versus ranibizumab, secondary analysis: fixed-effect analyses, Outcome 4 Gastrointestinal perforation.

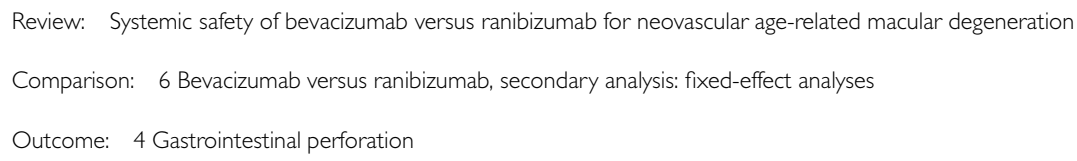

\begin{tabular}{ccccc} 
Study or subgroup & $\begin{array}{r}\text { Bevacizumab } \\
\mathrm{n} / \mathrm{N}\end{array}$ & $\begin{array}{c}\text { Ranibizumab } \\
\mathrm{n} / \mathrm{N}\end{array}$ & $\mathrm{M}-\mathrm{H}$, Fixed,95\% Cl & $\begin{array}{c}\text { Risk Ratio } \\
\mathrm{M}-\mathrm{H}, \mathrm{Fixed}, 95 \% \mathrm{Cl}\end{array}$ \\
\hline VIBERA & $0 / 107$ & $0 / 54$ & & Not estimable
\end{tabular}

Total (95\% CI)

107

54

Not estimable

Total events: 0 (Bevacizumab), 0 (Ranibizumab)

Heterogeneity: not applicable

Test for overall effect: not applicable

Test for subgroup differences: Not applicable

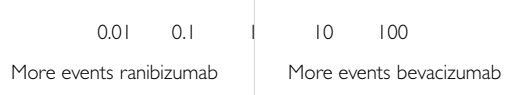


Analysis 6.5. Comparison 6 Bevacizumab versus ranibizumab, secondary analysis: fixed-effect analyses, Outcome 5 Infections.

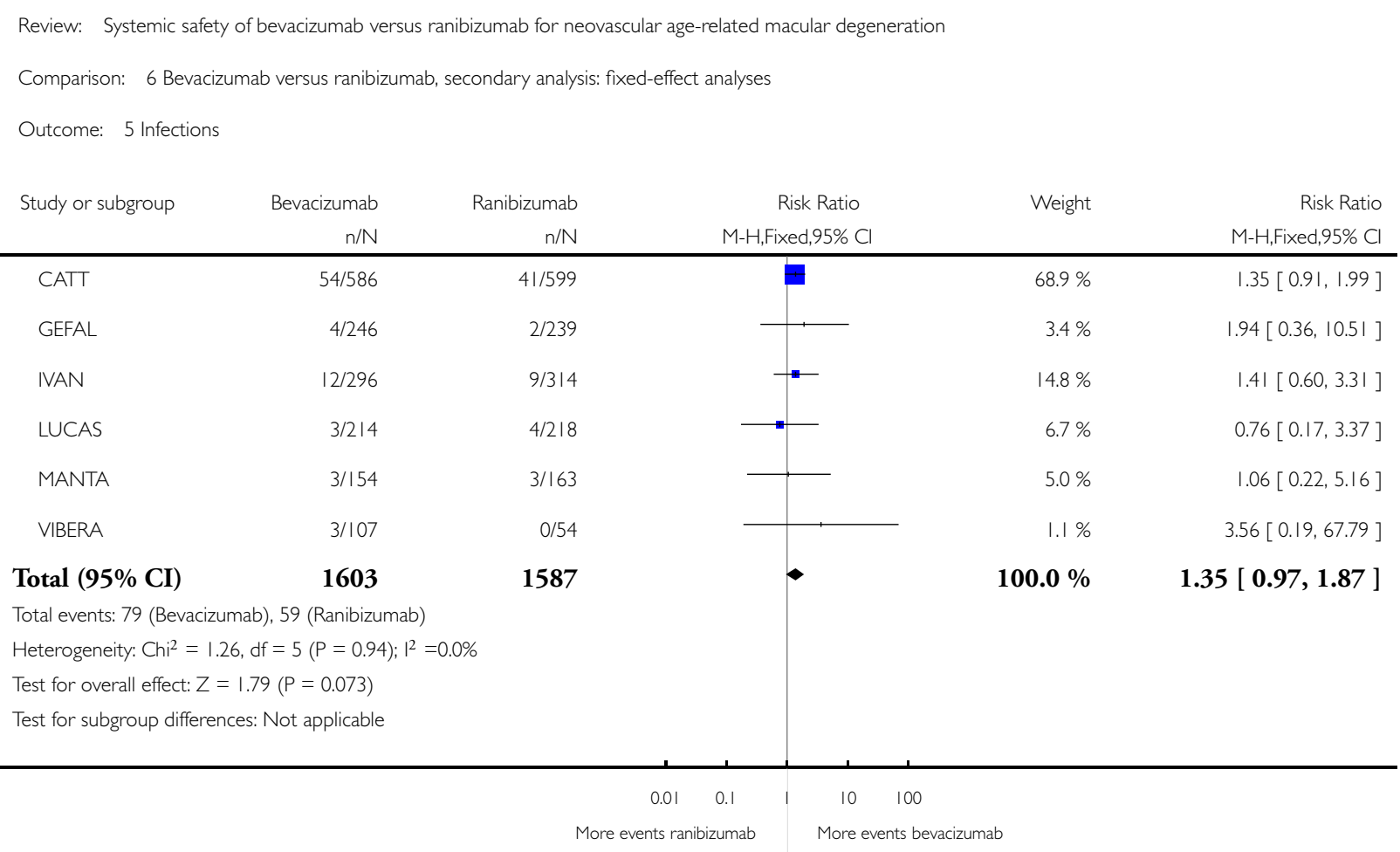


Analysis 6.6. Comparison 6 Bevacizumab versus ranibizumab, secondary analysis: fixed-effect analyses, Outcome 6 Myocardial infarction.

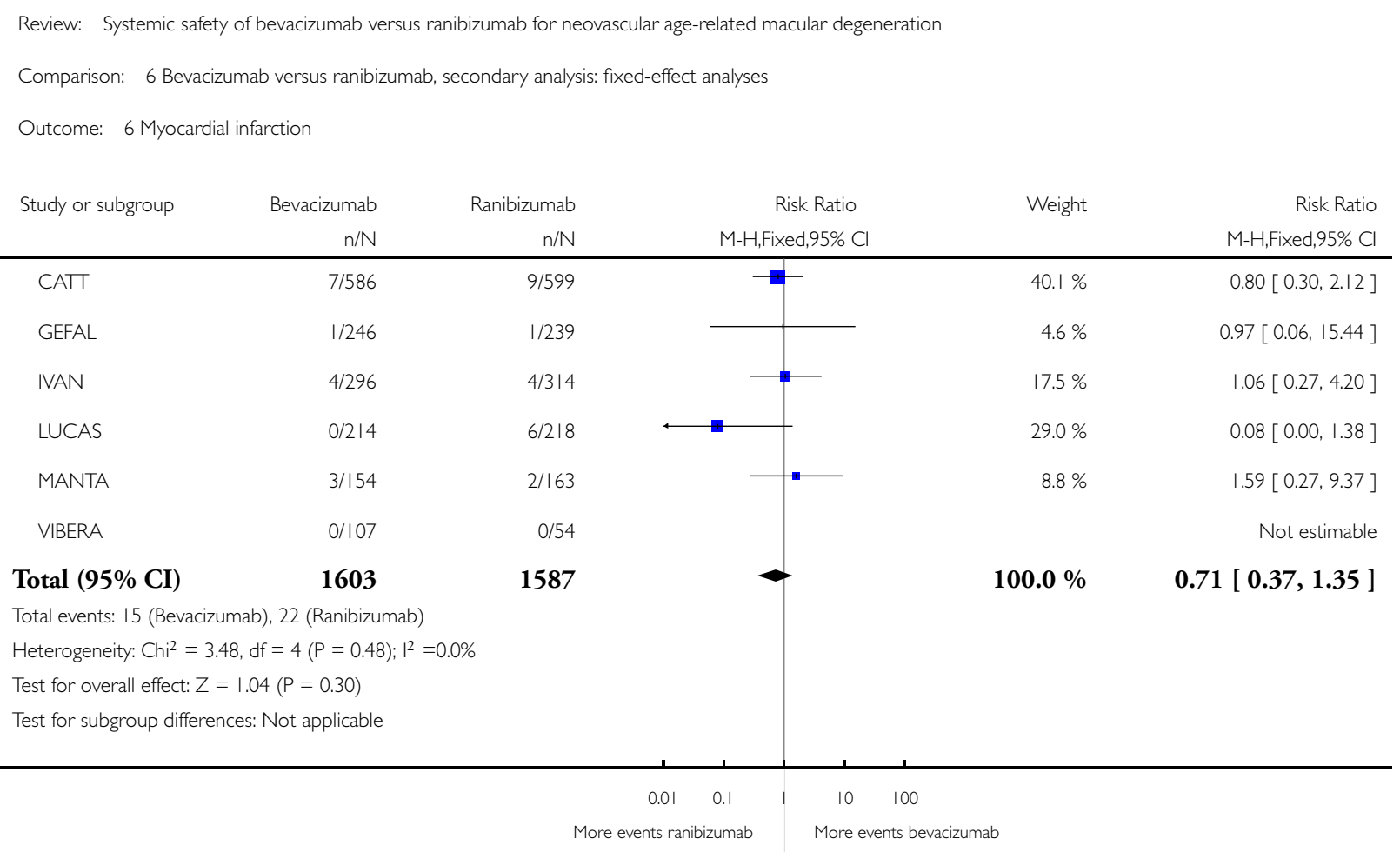


Analysis 6.7. Comparison 6 Bevacizumab versus ranibizumab, secondary analysis: fixed-effect analyses, Outcome 7 Neutropenia.

\begin{tabular}{|c|c|c|c|c|c|}
\hline \multicolumn{6}{|c|}{ Comparison: 6 Bevacizumab versus ranibizumab, secondary analysis: fixed-effect analyses } \\
\hline Outcome: 7 Neutr & & & & & \\
\hline \multirow[t]{2}{*}{ Study or subgroup } & Bevacizumab & Ranibizumab & Risk Ratio & Weight & Risk Ratio \\
\hline & $\mathrm{n} / \mathrm{N}$ & $\mathrm{n} / \mathrm{N}$ & \multicolumn{2}{|l|}{ M-H,Fixed,95\% Cl } & M-H,Fixed,95\% Cl \\
\hline VIBERA & 0/107 & $0 / 54$ & & & Not estimable \\
\hline Total $(95 \% \mathrm{CI})$ & 107 & 54 & & & Not estimable \\
\hline \multicolumn{6}{|c|}{ Total events: 0 (Bevacizumab), 0 (Ranibizumab) } \\
\hline \multicolumn{6}{|c|}{ Heterogeneity: not applicable } \\
\hline \multicolumn{6}{|c|}{ Test for overall effect: not applicable } \\
\hline \multicolumn{6}{|c|}{ Test for subgroup differences: Not applicable } \\
\hline
\end{tabular}

\section{Analysis 6.8. Comparison 6 Bevacizumab versus ranibizumab, secondary analysis: fixed-effect analyses, Outcome 8 Non-ocular haemorrhage.}

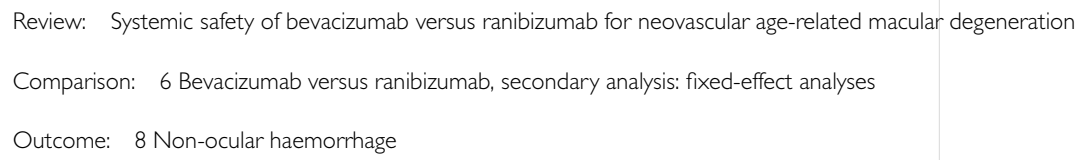


Analysis 6.9. Comparison 6 Bevacizumab versus ranibizumab, secondary analysis: fixed-effect analyses, Outcome 9 Stroke.

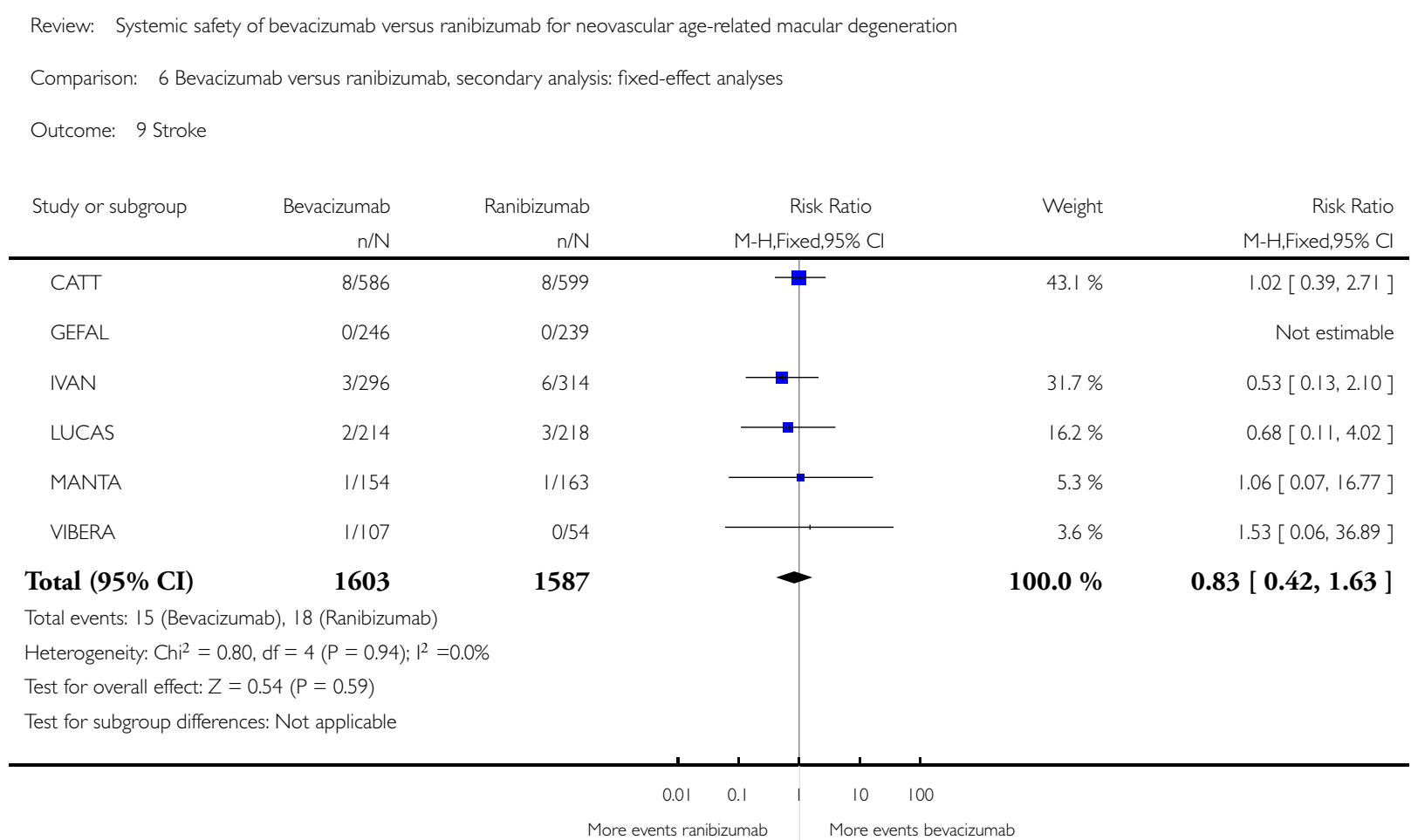


Analysis 6.1 I. Comparison 6 Bevacizumab versus ranibizumab, secondary analysis: fixed-effect analyses, Outcome I I Vascular events associated with anti-VEGF treatment.

Review: Systemic safety of bevacizumab versus ranibizumab for neovascular age-related macular degeneration

Comparison: 6 Bevacizumab versus ranibizumab, secondary analysis: fixed-effect analyses

Outcome: I Vascular events associated with anti-VEGF treatment

\begin{tabular}{|c|c|c|c|c|c|}
\hline \multirow[t]{2}{*}{ Study or subgroup } & Bevacizumab & Ranibizumab & Risk Ratio & Weight & Risk Ratio \\
\hline & $\mathrm{n} / \mathrm{N}$ & $\mathrm{n} / \mathrm{N}$ & M-H,Fixed,95\% Cl & & M-H,Fixed,95\% Cl \\
\hline CATT & $62 / 586$ & $45 / 599$ & + & $63.8 \%$ & $1.41[0.98,2.03]$ \\
\hline IVAN & $17 / 296$ & $26 / 314$ & $\because$ & $36.2 \%$ & $0.69[0.38,1.25]$ \\
\hline Subramanian 2010 & $0 / 20$ & $0 / 8$ & & & Not estimable \\
\hline Total (95\% CI) & 902 & 921 & $\bullet$ & $100.0 \%$ & $1.15[0.85,1.56]$ \\
\hline \multicolumn{6}{|c|}{ Total events: 79 (Bevacizumab), 7I (Ranibizumab) } \\
\hline \multicolumn{6}{|c|}{ Heterogeneity: $\mathrm{Chi}^{2}=3.99, \mathrm{df}=\mathrm{I}(\mathrm{P}=0.05) ; \mathrm{I}^{2}=75 \%$} \\
\hline \multicolumn{6}{|c|}{ Test for overall effect: $Z=0.89(P=0.37)$} \\
\hline Test for subgroup diffe & Vot applicable & & & & \\
\hline
\end{tabular}

More events ranibizumab More events bevacizumab 
Analysis 6.12. Comparison 6 Bevacizumab versus ranibizumab, secondary analysis: fixed-effect analyses, Outcome 12 Cardiac disorders MedDRA Class.

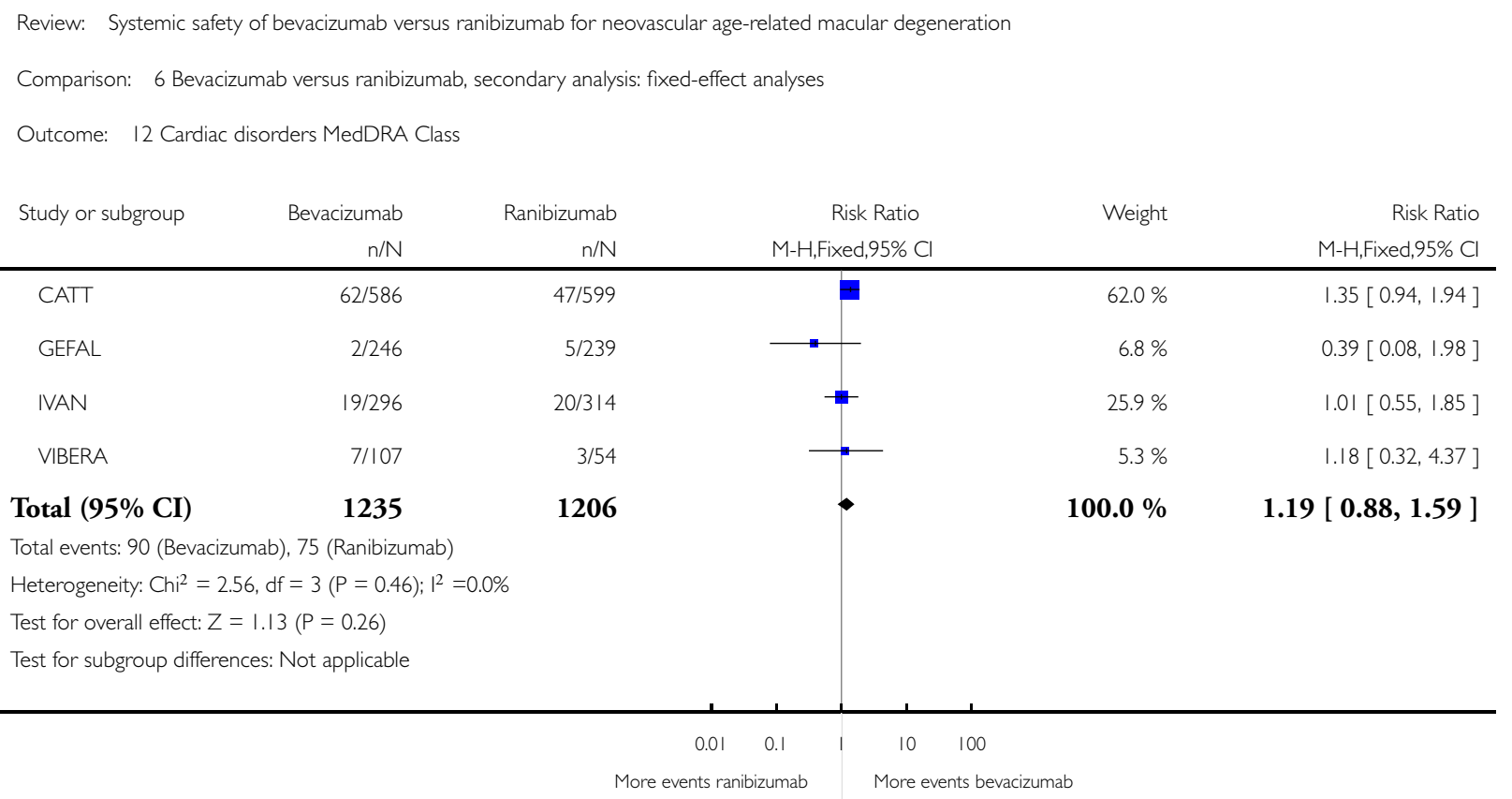


Analysis 6.13. Comparison 6 Bevacizumab versus ranibizumab, secondary analysis: fixed-effect analyses, Outcome 13 Gastrointestinal disorders MedDRA Class.

Review: Systemic safety of bevacizumab versus ranibizumab for neovascular age-related macular degeneration

Comparison: 6 Bevacizumab versus ranibizumab, secondary analysis: fixed-effect analyses

Outcome: 13 Gastrointestinal disorders MedDRA Class

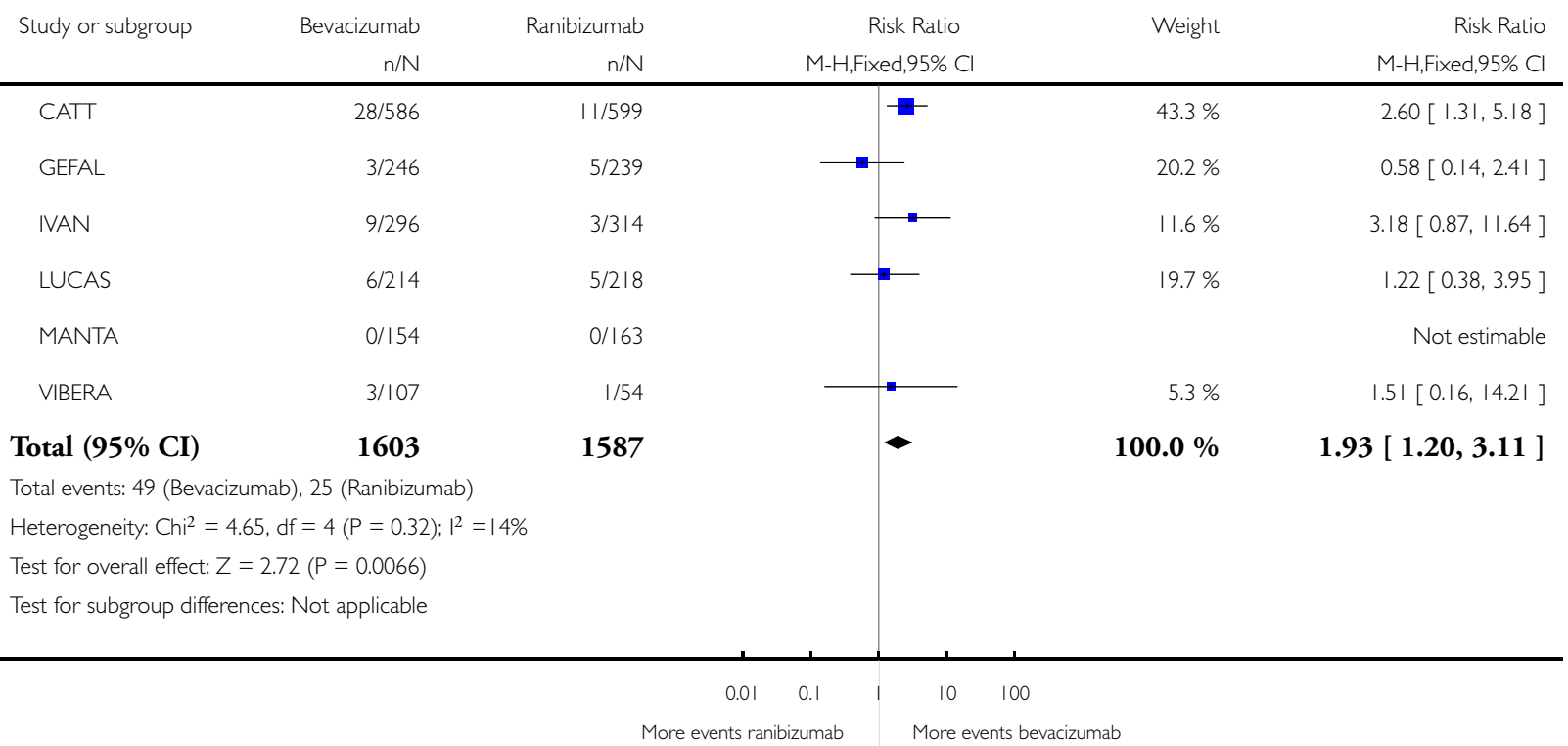


Analysis 6.14. Comparison 6 Bevacizumab versus ranibizumab, secondary analysis: fixed-effect analyses, Outcome 14 General disorders and administration site conditions MedDRA Class.

Review: Systemic safety of bevacizumab versus ranibizumab for neovascular age-related macular degeneration

Comparison: 6 Bevacizumab versus ranibizumab, secondary analysis: fixed-effect analyses

Outcome: 14 General disorders and administration site conditions MedDRA Class

\begin{tabular}{|c|c|c|c|c|c|}
\hline \multirow[t]{2}{*}{ Study or subgroup } & Bevacizumab & Ranibizumab & Risk Ratio & Weight & Risk Ratio \\
\hline & $\mathrm{n} / \mathrm{N}$ & $\mathrm{n} / \mathrm{N}$ & M-H,Fixed,95\% Cl & & M-H,Fixed,95\% Cl \\
\hline GEFAL & $4 / 246$ & $0 / 239$ & $\longrightarrow$ & $3.4 \%$ & $8.74[0.47,161.55]$ \\
\hline IVAN & $16 / 296$ & $|5 / 3| 4$ & & $96.6 \%$ & $1.13[0.57,2.25]$ \\
\hline VIBERA & $0 / 107$ & $0 / 54$ & & & Not estimable \\
\hline Total (95\% CI) & 649 & 607 & & $100.0 \%$ & $1.39[0.73,2.65]$ \\
\hline \multicolumn{6}{|c|}{ Total events: 20 (Bevacizumab), I5 (Ranibizumab) } \\
\hline \multicolumn{6}{|c|}{ Heterogeneity: $\mathrm{Chi}^{2}=1.87, \mathrm{df}=\mathrm{I}(\mathrm{P}=0.17) ; \mathrm{I}^{2}=47 \%$} \\
\hline \multicolumn{6}{|c|}{ Test for overall effect: $Z=0.99(P=0.32)$} \\
\hline Test for subgroup diff & Not applicable & & & & \\
\hline
\end{tabular}

More events ranibizumab More events bevacizumab 
Analysis 6.15. Comparison 6 Bevacizumab versus ranibizumab, secondary analysis: fixed-effect analyses, Outcome 15 Neoplasms benign, malignant, and unspecified MedDRA Class.

Review: Systemic safety of bevacizumab versus ranibizumab for neovascular age-related macular degeneration

Comparison: 6 Bevacizumab versus ranibizumab, secondary analysis: fixed-effect analyses

Outcome: 15 Neoplasms benign, malignant, and unspecified MedDRA Class

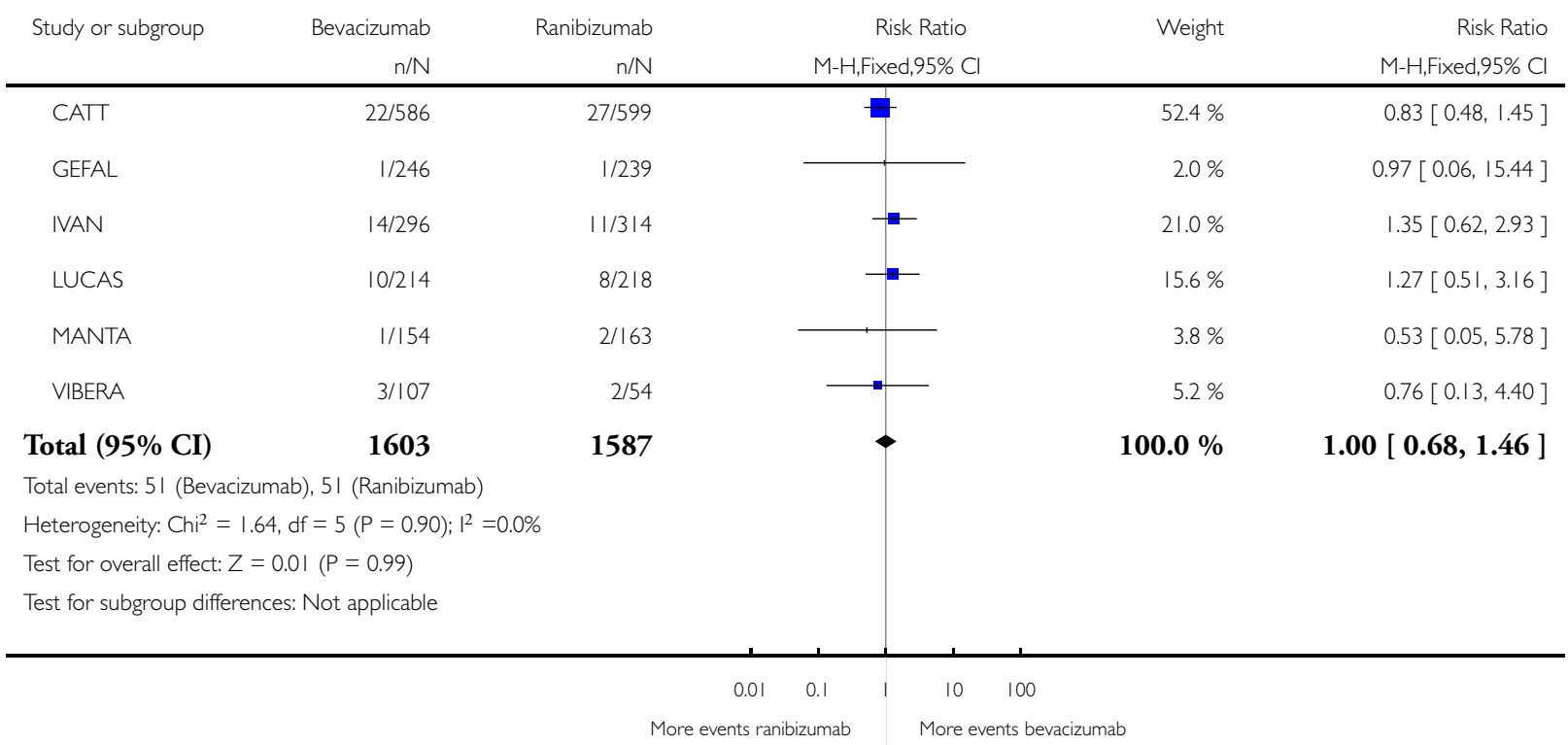


Analysis 6.16. Comparison 6 Bevacizumab versus ranibizumab, secondary analysis: fixed-effect analyses, Outcome 16 Nervous system disorders MedDRA Class.

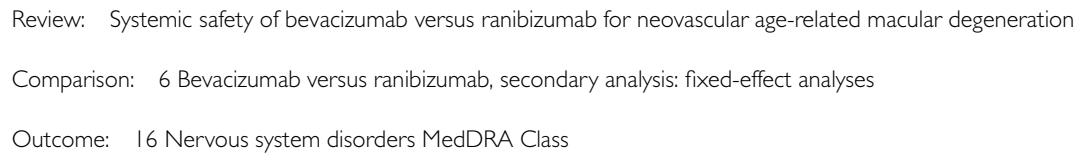

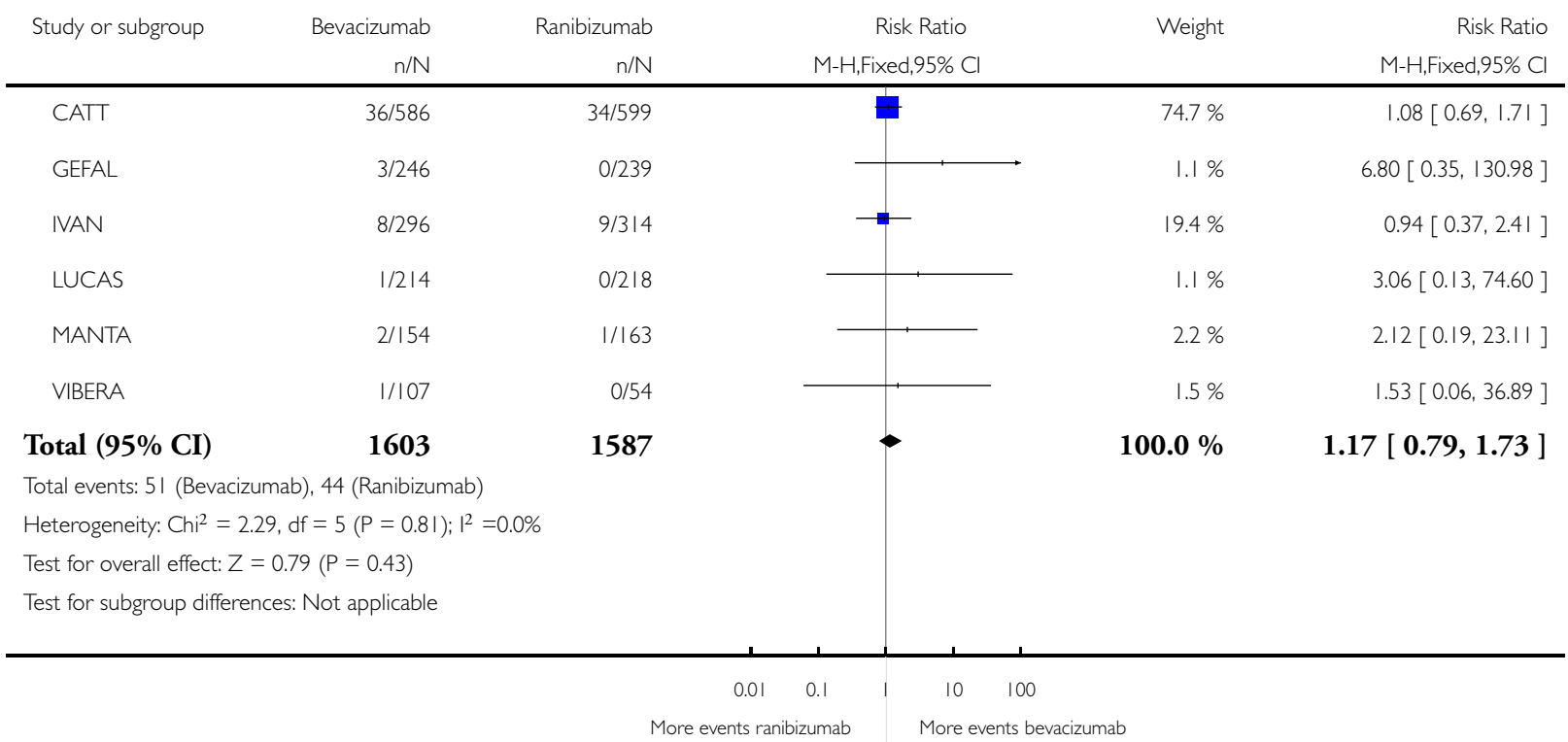


Analysis 6.17. Comparison 6 Bevacizumab versus ranibizumab, secondary analysis: fixed-effect analyses, Outcome 17 Respiratory, thoracic, and mediastinal disorders MedDRA Class.

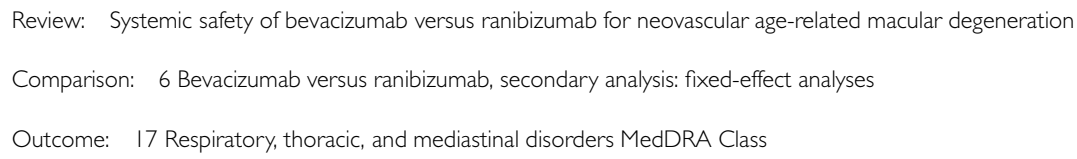

\begin{tabular}{|c|c|c|c|c|c|}
\hline \multirow[t]{2}{*}{ Study or subgroup } & Bevacizumab & Ranibizumab & Risk Ratio & Weight & Risk Ratio \\
\hline & $\mathrm{n} / \mathrm{N}$ & $\mathrm{n} / \mathrm{N}$ & M-H,Fixed,95\% Cl & & M-H,Fixed,95\% Cl \\
\hline GEFAL & $4 / 246$ & $4 / 239$ & & $34.3 \%$ & $0.97[0.25,3.84]$ \\
\hline IVAN & $7 / 296$ & $8 / 314$ & . & $65.7 \%$ & $0.93[0.34,2.53]$ \\
\hline VIBERA & $0 / 107$ & $0 / 54$ & & & Not estimable \\
\hline
\end{tabular}

Total (95\% CI)

649

607

$100.0 \%$

$0.94[0.42,2.12]$

Total events: I I (Bevacizumab), 12 (Ranibizumab)

Heterogeneity: $\mathrm{Chi}^{2}=0.00, \mathrm{df}=\mathrm{I}(\mathrm{P}=0.96) ; \mathrm{I}^{2}=0.0 \%$

Test for overall effect: $Z=0.14(P=0.89)$

Test for subgroup differences: Not applicable

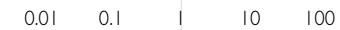

More events ranibizumab More events bevacizumab

\section{CONTRIBUTIONSOFAUTHORS}

Study concept: RD, LM, GV.

Study protocol: VB, AC, UC, KD, RD, KK, LK, EL, KL, YL, AM, BM, DM, LM, MM, IP, BR, CR, CS, MS, GV.

Search strategy: CS, GV.

Selection of studies: CS, GV.

Provision of data (i.e., trialists): UC, LK, BM, DM, MM, IP, BR, CR, MS.

Acquisition of data: KK, EL, GV, LM.

'Risk of bias' assessment: KK, EL, GV, LM.

Analysis of data: KK, EL, LM, GV.

Drafting of the manuscript: KK, EL, LM, GV.

Interpretation and critical revision of the manuscript for important intellectual content: VB, AC, UC, KD, RD, KK, LK, EL, KL, YL, AM, BM, DM, LM, MM, IP, BR, CR, CS, MS, GV. 


\section{DECLARATIONSOF INTEREST}

Some of the review authors are trial investigators on the studies included in this review.

\section{SOURCES OF SUPPORT}

\section{Internal sources}

- No sources of support supplied

\section{External sources}

- Emilia Romagna Regional Health Service, Italy.

This systematic review has been funded by the Emilia Romagna Regional Health Service to the Italian Cochrane Centre. The funding source had no role in the development of the systematic review or the writing of this manuscript.

- Italian Ministry of Health, Italy.

Lorenzo Moja is a recipient of a Research Early Career Award from the Italian Ministry of Health on "Improving appropriateness and transparency of processes to develop guidelines on controversial clinical areas: evidence, values and context preferences to help mitigate disputes and enhance the applicability of recommendations to practice" (GR-2011-02348048).

- National Institutes of Health, USA.

- Dr. Maguire's effort in contributing to the design and reporting of this review was supported by a grant (U10-EY017823) from the National Eye Institute, National Institutes of Health, Department of Health and Human Services, United States.

- Kay Dickersin's contribution was supported by Grant 1 (U01-EY020522) from the National Eye Institute, National Institutes of Health.

National Institute for Health Research (NIHR), UK.

- Richard Wormald, Co-ordinating Editor for the Cochrane Eyes and Vision Group (CEVG) acknowledges financial support for his CEVG research sessions from the Department of Health through the award made by the National Institute for Health Research to Moorfields Eye Hospital NHS Foundation Trust and UCL Institute of Ophthalmology for a Specialist Biomedical Research Centre for Ophthalmology.

- The NIHR also funds the CEVG Editorial Base in London.

The views expressed in this publication are those of the authors and not necessarily those of the NIHR, NHS, or the Department of Health.

\section{DIFFERENCES BETWEEN PROTOCOLANDREVIEW}

There are no differences between the protocol and the review. 